\title{
Autonomous Navigation of Unmanned Aerial Vehicles Subjected to Time Delays
}

\author{
by \\ Walter Aburime, B.Eng \\ A thesis submitted to the \\ Faculty of Graduate and Postdoctoral Affairs \\ in partial fulfillment of the requirements for the degree of
}

Master of Applied Science in Electrical Engineering

Ottawa-Carleton Institute for Electrical and Computer Engineering

Department of Systems and Computer Engineering

Carleton University

Ottawa, Ontario

August, 2018

(C) Copyright

Walter Aburime, 2018 
The undersigned hereby recommends to the

Faculty of Graduate and Postdoctoral Affairs acceptance of the thesis

\title{
Autonomous Navigation of Unmanned Aerial Vehicles Subjected to Time Delays
}

\author{
submitted by Walter Aburime, B.Eng \\ in partial fulfillment of the requirements for the degree of
}

Master of Applied Science in Electrical Engineering

Professor Howard Schwartz, Thesis Supervisor

Professor Sidney Givigi, Thesis Co-supervisor

Professor Yvan Labiche, Chair,

Department of Systems and Computer Engineering

Ottawa-Carleton Institute for Electrical and Computer Engineering

Department of Systems and Computer Engineering

Carleton University

August, 2018 


\section{Abstract}

This thesis presents a new method of compensating for time delays in the control and navigation of UAVs. The aerial vehicles are controlled with a coordinated lateral control. The operator signals are delayed and a bank of recursive least squares (RLS) filters are used to identify the delay and the target waypoint. Hypothesis testing is implemented to select the filter that most closely matches the delay. This filter determines the delay and the target waypoint.

Once a filter is selected, the UAV then computes its heading to the estimated target waypoint. By executing the self computed heading, the UAV performs autonomous navigation to the target waypoint. The operator keeps operating the UAV

and the UAV keeps track of the operator commands so that if there is a change in delay or the waypoint, the UAV learns and adjusts accordingly. 
To my Family. 


\section{Acknowledgments}

My deepest gratitude to my thesis supervisor Prof. Howard Schwartz for his patience and guidance in this thesis. To you I say a very big thank you for always making yourself available to answer my questions and dispel my confusions. I like to thank my co-supervisor Prof. Givigi for all the support and guidance, this will not have been complete without your inputs.

I want to express my appreciation to my family who have supported me in everything.

And to my Wife, words fail me. ThankYou!!!!!! 


\section{Table of Contents}

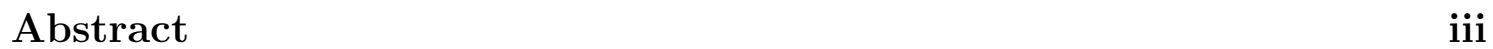

\begin{tabular}{ll}
\hline Acknowledgments & v
\end{tabular}

Table of Contents vi

List of Figures viii

\begin{tabular}{lll}
\hline 1 & Introduction & 1
\end{tabular}

1.1 UAV and Time Delays . . . . . . . . . . . . . . . . . . . . 1

1.1 .1 Time Delays and Effects of Time Delays . . . . . . . . . . 3

1.1 .2 Previous Work and Literature Review . . . . . . . . . . . . . . 4

1.2 Motivation . . . . . . . . . . . . . . . . . . . . . 6

1.3 Contributions . . . . . . . . . . . . . . . . . 6

\begin{tabular}{|lll}
\hline 2 & Aircraft Dynamics and Control & 8
\end{tabular}

2.1 Lateral Control of an Aircraft . . . . . . . . . . . . . . . . 8

2.1 .1 Pole Placement . . . . . . . . . . . . . . . . . . . . . . 12

2.1 .2 Controller Design $\ldots \ldots \ldots \ldots \ldots$

2.2 Time Delays $\ldots \ldots \ldots \ldots \ldots$




$3.1 \quad$ Brief Review of Least Squares(LS) and Recursive Least Square(RLS) Estimation . . . . . . . . . . . . . . . . . . . . . . . . . . 29

$3.1 .1 \quad$ Least Squares(LS) Estimate . . . . . . . . . . . . . . . . . 29

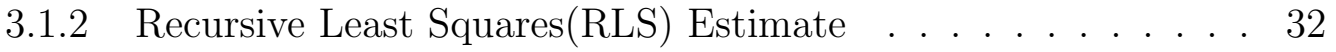

$3.2 \quad$ How to apply RLS to Delay Identification $\ldots \ldots \ldots \ldots$

3.2 .1 Bank of Least Squares Filters . . . . . . . . . . . . . . 47

3.2 .2 Simulation results: . . . . . . . . . . . . . . . . . 51

3.2 .3 Bank of Recursive Least Squares Filters . . . . . . . . . . 58

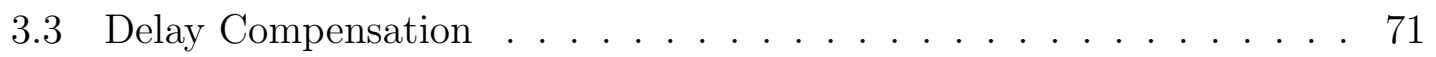

$\begin{array}{|lll|}4 & \text { Hypothesis testing and Delay Compensation } & 73\end{array}$

4.1 Introduction to Hypothesis Testing for Filter Selection . . . . . . . . 73

4.1.1 Demonstration of filter selection using Hypothesis testing . . . 79

$4.2 \quad$ Delay Compensation $\ldots \ldots \ldots \ldots$

\begin{tabular}{|lll}
\hline 5 & Conclusion and Future Work & 116
\end{tabular}

5.1 Conclusion . . . . . . . . . . . . . . . . . . . . . . . . 116

$5.1 .1 \quad$ Future Work. . . . . . . . . . . . . . . . . . . . . . . . 117

\begin{tabular}{ll}
\hline List of References & 118
\end{tabular} 


\section{List of Figures}



1.2 UAVs with different applications $\ldots \ldots \ldots \ldots$

1.3 Tele-operators at a ground center station $\ldots \ldots \ldots \ldots$

$2.1 \quad$ Aircraft Control Surface $\ldots \ldots \ldots \ldots$

2.2 Yaw and Sideslip angle $\ldots \ldots \ldots \ldots$

$2.3 \quad$ Aircraft system $\ldots \ldots \ldots \ldots \ldots \ldots$

$2.4 \quad$ Roll angle $\ldots \ldots \ldots \ldots$

$2.5 \quad$ Unit step response of $\rho, \mathrm{r}, \phi, \psi$ with poles placed at $-1,-1 \pm 3 j \ldots 14$

$2.6 \quad$ Unit step response $\ldots \ldots \ldots \ldots$

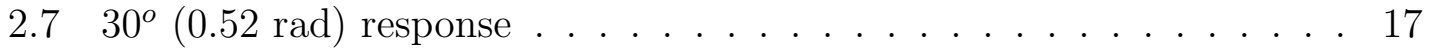

$2.8 \quad 45^{\circ}(0.785 \mathrm{rad})$ response $\ldots \ldots \ldots \ldots \ldots \ldots$

$2.960^{\circ}(1.047 \mathrm{rad})$ response $\ldots \ldots \ldots \ldots \ldots$

2.10 Unit step response of $\rho, \mathrm{r}, \phi, \psi . \ldots \ldots \ldots \ldots$

2.11 Rudder angle . . . . . . . . . . . . . . . . . . . . . 20

2.12 Aileron angle. . . . . . . . . . . . . . . . . . . . 20

2.13 Aircraft trajectory subjected to a $30^{\circ}$ heading command. . . . . . . . 21

2.14 Aircraft trajectory subjected to a $45^{\circ}$ heading command. . . . . . . . 22

2.15 Aircraft trajectory, no delay . . . . . . . . . . . . . . . . 23

2.16 Unit step response with a delayed input command. . . . . . . . . . . 24

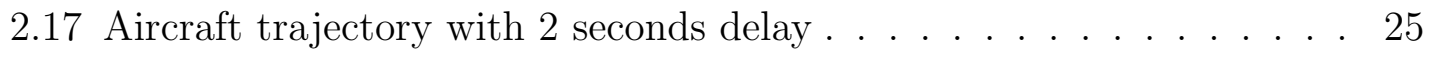

2.18 Aircraft trajectory with 5 seconds delay $\ldots \ldots \ldots$ 


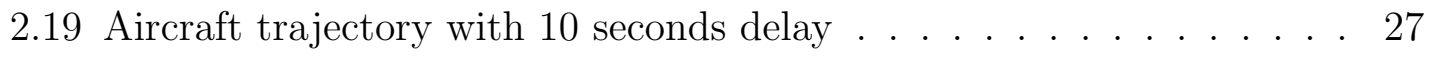

2.20 Aircraft trajectory with 20 seconds delay $\ldots \ldots \ldots \ldots$

2.21 Aircraft trajectory with 30 seconds delay $\ldots \ldots \ldots$. . . . . 28

$3.1 \quad$ UAV position $\ldots \ldots \ldots \ldots \ldots \ldots$

3.2 Example $3.1 \ldots \ldots \ldots \ldots$

3.3 Example $3.2 \ldots \ldots \ldots \ldots$

$3.4 \quad$ UAV location in different heading . . . . . . . . . . . . . . . 42

$3.5 \quad$ Points at different time steps $t$ on the UAV trajectory . . . . . . . . 43

$3.6 \quad$ UAV trajectory with 2s delayed input commands as it moves from waypoint $A$ to $F$. The red dotted line indicates the aircraft trajectory with no delay, the blue line is the straight line between waypoints and the black line indicates the UAV trajectory subjected to time delay. . 50

3.7 Least squares waypoint estimate of the 5 step LS Filter . . . . . . . . 52

3.8 The prediction errors of the 5 step LS Filter . . . . . . . . . . . 52

3.9 Least squares waypoint estimate of the 10 step (1 second) LS Filter . 53

3.10 The prediction errors of the 10 step LS Filter $\ldots \ldots \ldots$. . . . . 53

3.11 Least squares waypoint estimate of the 15 step (1.5 seconds) LS Filter 54

3.12 The prediction errors of the 15 step LS Filter $\ldots \ldots \ldots$. . . . 54

3.13 Least squares waypoint estimate of the 20 step (2 seconds)LS Filter . 55

3.14 The prediction errors of the 20 step LS Filter . . . . . . . . . 55

3.15 Least squares waypoint estimate of the waypoint. . . . . . . . . 56

3.16 Least squares waypoint estimate of the waypoint. . . . . . . . . . 56

3.17 The prediction errors of the four LS Filter $\ldots \ldots \ldots$. . . . 57

3.18 Target waypoint estimate of the 5 step RLS Filter . . . . . . . . . 60

3.19 Prediction Errors of the 5 step RLS Filter. . . . . . . . . . . . 61

3.20 Target waypoint estimate of the 10 step RLS Filter $\ldots \ldots$. . . . . 61

3.21 Prediction Errors of the 10 step RLS Filter. . . . . . . . . . . . 62 
3.22 Target waypoint estimate of the 15 step RLS Filter . . . . . . . . . . 62

3.23 Prediction Errors of the 15 step RLS Filter. . . . . . . . . . . . . . . 63

3.24 Target waypoint estimate of the 20 step RLS Filter . . . . . . . . . . 63

3.25 Prediction Errors of the 20 step RLS Filter. . . . . . . . . . . . . . . 64

$3.26 x_{w p}$ RLS estimate of the target waypoint . . . . . . . . . . . . 65

$3.27 y_{w p}$ RLS estimate of the target waypoint . . . . . . . . . . . . . 66

3.28 Prediction errors of the four RLS filters. . . . . . . . . . . . . . . . . 67

3.29 Prediction errors of the four RLS filters for 0 to 700 time steps. . . . 68

3.30 Prediction errors of the four RLS filters for 700 to 1150 time steps. . . 69

3.31 Prediction errors of the 20 step RLS filters for 700 to 1150 time steps. 70

3.32 UAV trajectory for a 10 s delay compensation . . . . . . . . . . . . . 72

4.1 Two tailed t-test at a $\alpha$ level of significance. . . . . . . . . . . . . . . 76

$4.2 \quad$ Two tailed t-test within a user-defined rejection threshold Tr. . . . . 78

$4.3 \quad$ Hypothesis test selection of 5 step RLS filter . . . . . . . . . . . . . . 81

4.4 T-test statistic of 5 (red), 10 (blue), 15 (magenta) and 20 (black) step RLS filters. . . . . . . . . . . . . . . . . . . . . . . . . . 82

4.5 Hypothesis test selection of 10 step RLS filter $\ldots . . . . . .83$

$4.6 \quad$ T-test statistic of 5 (red), 10 (blue), 15 (magenta) and 20 (black) step RLS filters. . . . . . . . . . . . . . . . . . . . . 84

$4.7 \quad$ Hypothesis test selection of 15 step RLS filter . . . . . . . . . . . . . 85

4.8 T-test statistic of 5 (red line), 10 (blue line), 15 (magenta line) and 20 (black line) step RLS filters. . . . . . . . . . . . . . . . . . 86

4.9 Hypothesis test selection of 20 step RLS filter . . . . . . . . . . . . . 87

4.10 T-test statistic of 5 (red), 10 (blue), 15 (magenta) and 20 (black) step RLS filters. . . . . . . . . . . . . . . . . . . . . . . 88

4.11 Hypothesis test selection RLS filters in sequence A . . . . . . . . . . 89 
4.12 UAV trajectory when the errors go through zero at $t=840$. The red dotted line indicates the aircraft trajectory with no delay, the blue line is the straight line between waypoints and the black line indicates the UAV trajectory subjected to time delay sequence. . . . . . . . . . 91

4.13 T-test statistic $t_{\text {stat }}$ of the 5 (red), 10 (blue), 15 (magenta) and 20 (black) step RLS filters in time delay sequence A . . . . . . . . . . . 92

4.14 Hypothesis test selection RLS filters in sequence B. . . . . . . . . . . 93

4.15 T-test statistic $t_{\text {stat }}$ of the 5 (red), 10 (blue), 15 (magenta) and 20 (black) step RLS filters in time delay sequence B . . . . . . . . . . . 94

4.16 Hypothesis test selection RLS filters in sequence C . . . . . . . . . . 95

4.17 T-test statistic $t_{\text {stat }}$ of the 5 (red), 10 (blue), 15 (magenta) and 20 (black) step RLS filters in time delay sequence C . . . . . . . . . . . 96

4.18 Hypothesis test selection RLS filters in example 1 . . . . . . . . . . . 98

4.19 T-test statistic $t_{\text {stat }}$ of RLS filters in example 1 . . . . . . . . . . . . . 99

4.20 Hypothesis test selection of 10 step RLS filter for a 0.8 seconds timed delay . . . . . . . . . . . . . . . . . . . . . . 100

4.21 T-test statistic $t_{\text {stat }}$ of RLS filters in example 2 . . . . . . . . . . . . . 101

4.22 Hypothesis test selection of RLS filters in example 3 . . . . . . . . . . 102

4.23 T-test statistic $t_{\text {stat }}$ in example 3 . . . . . . . . . . . . . . 103

4.24 Final output of hypothesis test selection of RLS filters in Example 3.104 4.25 Modified Aircraft system for autonomous navigation. . . . . . . . . . 106 4.26 UAv trajectory with 10 seconds time delay . . . . . . . . . . . . . . . 107

4.27 Hypothesis test selection of the 200 step delay RLS filter. . . . . . . . . 108

4.28 Compensation for a 20 seconds delay in input commands . . . . . . . 109

4.29 Hypothesis test selection of the 300 step delay RLS filter. . . . . . . . . 110

4.30 Compensation for a 30 seconds delay in input commands . . . . . . . 111

4.31 Hypothesis test selection of the 600 step delay RLS filter. . . . . . . . 112 
4.32 Compensation for a 60 seconds delay in input commands . . . . . . . 113

4.33 Compensation for a 10 seconds delay in input commands . . . . . . . 114 


\section{Chapter 1}

\section{Introduction}

\subsection{UAV and Time Delays}

Unmanned aerial vehicles (UAVs), also known as drones, refer to any flying machines operated without on-board pilots. They come in different shapes, sizes, forms and types. They were first used by the military in 1917 during the World War I and since then UAV applications have grown enormously in both the military and civilian sectors 11. In the military, UAVs are used for Intelligence gathering, Surveillance, Target Acquisition and Reconnaissance (ISTAR) .In Agriculture, UAVs are used in farms to increase productivity, in Health care for several life saving missions, in Media for photography and filming.

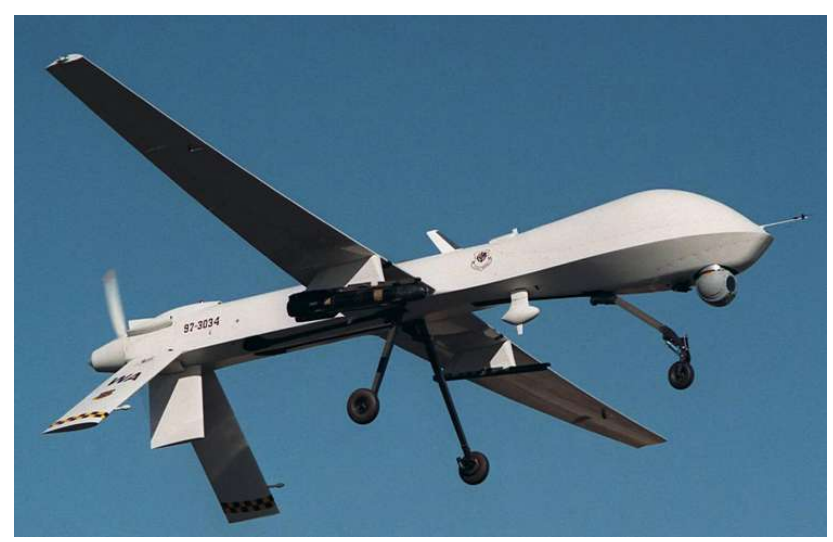

Figure 1.1: MQ-1 Predator combat drone 


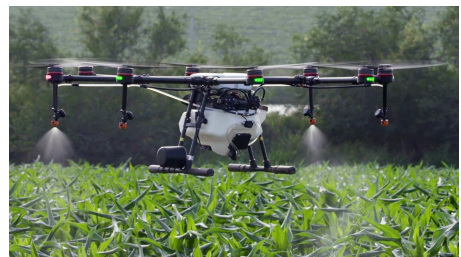

(a) Agricultural Wonder Drone

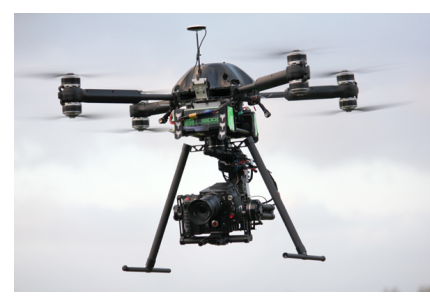

(b) Media Drone

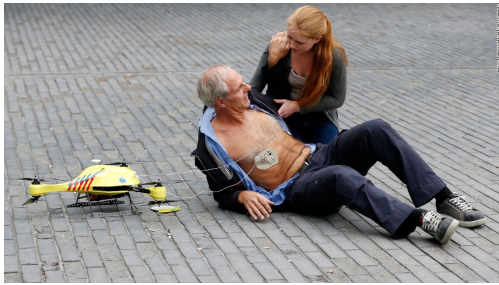

(c) Medicine Drone

Figure 1.2: UAVs with different applications

UAVs are either completely autonomous or remotely controlled from a distance by a human operator (this is referred to as teleoperation). In teleoperation, the human operator known as the teleoperator, remotely generates commands which are transmitted to the UAV to control and guide the UAV in performing tasks. Many UAV applications involve long distance and some applications have been reported to involve intercontinental teleoperation. In 2017, A.Y Mersha piloted a UAV in Australia from his control station in the Netherlands 2.

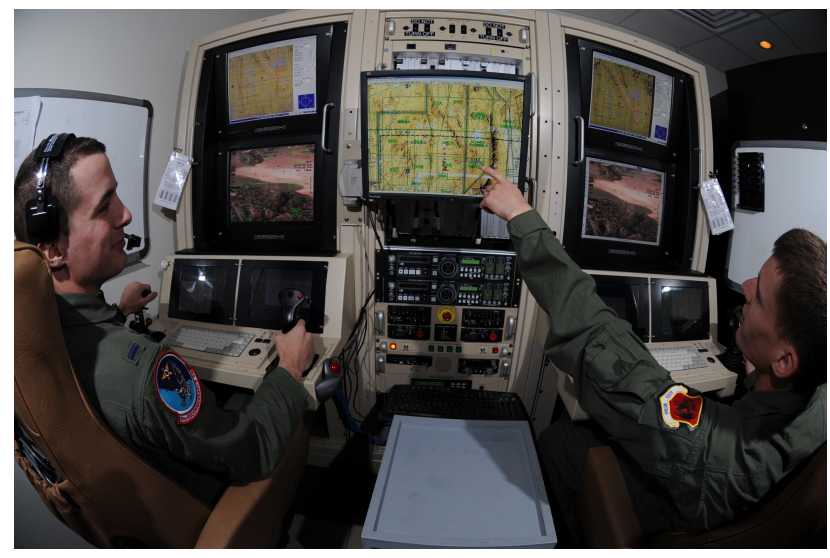

Figure 1.3: Tele-operators at a ground center station

The teleoperator commands are transmitted over the distance of separation between the operator and UAV. This separation creates a negative impact on the performance of the UAV. This negative impact could be a result of unforeseen environmental constraints and disturbances like wind, signal transmission loss and signal 
delay as signals travel over geographical distances and between various transceivers. These are some factors that contribute to time delays in UAV response to teleoperator commands.

\subsubsection{Time Delays and Effects of Time Delays}

Time delay is the wait time between when the operator sent a control signal and when the UAV responds to the signal. A signal sent at any time $t$, will travel the distance of separation in the prevailing conditions before it reaches the UAV at time $t+$ timedelay. The time delay is simply the time it takes for the control signal to get to the UAV. Time delays reduce the operational accuracy and quality of the UAV particularly in operations that require precise knowledge of the UAVs location.

The effects of time delays in UAV systems have been an active research area and is still ongoing. Many articles have documented the effects of time delay on UAV systems and operator performance. In 3 the effects of time delay on the control of the UAV were investigated. It was observed that there is a maximum time delay within which the system remained stable but an increase beyond this delay would make the system unstable. The author also recorded observations of time delay effects at different target speeds. The author recommends that UAVs subjected to time delay should be operated at slow speeds.

In 44 the author records how time delay affects operator performance and the difficulty of executing remote manipulative tasks. The evidence shows that a system subjected to time delays, will increase task difficulty and consequently increase task completion time.

Time delay was observed to affect the landing quality of UAVs in 5 and UAV target reach performance. It was observed that time delays increase aircraft deviation from target glide-scope and poor landing positioning at the landing threshold.

In 6 time delays were investigated on combat UAVs (known as UCAV) to see 
the effect on mission performance. It was discovered that errors in control depend on time delay. Delays of 30 seconds were found to be operationally unacceptable and even delays as small as 300 miliseconds in communicaton between the UCAV and teleoperator were found to cause targets misses.

The research work highlighted in 3 4 5 6, provide some experimental evidence on the effect of time delay in UAV control and performance. Time delay has been shown to cause increased difficulty of control tasks, poor operator performance, system instability leading to unsafe teleoperation, poor situation awareness, poor efficiency of teleoperation, and vehicle control impairment.

Teleoperated UAVs are faced with the challenges of time delays. This has also affected the acceptability of UAVs because a lot of mishaps and accidents involving UAVs have been attributed to delayed UAV response.

\subsubsection{Previous Work and Literature Review}

The research community have been working to offer solutions to the issue of time delays. Predictive control methods were used to generate a sequence of future control inputs at different conditions 7 8, 9, 10. The UAV then selects a suitable input that corresponds to prevailing conditions, assuming that the UAV follows a linear dynamic model.

A machine learning technique known as Gaussian Process (GP) in 10 is proposed to compensate communication delays between teleoperator and UAV. However this technique does not learn the delay but learns the pattern of networked induced effects of UAV manoeuvres.

In 11] 12, digital signal processing techniques and a set of previous system data were used to predict future positions of the UAV to compensate for time delay. The method is known as Algorithmic Predictions (AP). In 11] 12, comparison was made between Algorithmic Predictions and Perceptual Adaptation (PA). In 
perceptual adaptation, tracking strategies are modified to adapt to the time delay. Perceptual adaptation offered a natural compensation for time delay. However, the predictive method of Algorithmic prediction proved superior because the root mean square error in AP decreased while there was a slight increase in PAs rms error.

The work in 4 indicates that delay can be compensated for at the expense of time by a method termed the move and wait strategy. The move and wait strategy involves performing manipulative tasks with a delay by making an openloop movement at the Ground Control Station(GCS), waiting until the remote hand responds at the UAV site, and repeating this process until the necessary accuracy has been achieved.

As the varied applications of UAVs increases, so does the need for accuracy of system performance and delivery. The smallest of time delays may lead to difficulty in UAV control depending on the design complexity particularly in operations that require a high level of UAV target reach accuracy. In health care for instance, when a life saving service is required at a target location, delays in a UAV may cause the UAV to miss its target leading to possible loss of life and crash. The control performance of UAVs are limited by time delays and it is therefore imperative that time delays are accurately estimated and managed to avoid unfortunate circumstances.

This thesis presents a method of time delay compensation in the navigation of UAVs over a known range of time delays. We propose a method of learning the time delay and the destination of the UAV using past UAV coordinates and respective operator commanded headings on the UAV's trajectory. This method is particularly useful because it is not dependent on the prevailing conditions or the speed of the UAV. The methods in 7, 8, 9 and 10 aim at time delay compensation using predictive methods to estimate future states and define actions based on the predicted future states, but the methods do not learn the target waypoint. The proposed method in this thesis learns the target waypoint and then learns the delay. 
Once the target waypoint and delay is learned, the UAV then compensates for the learned delay by self navigation. Also, with the proposed method in this thesis there is a higher level of accuracy with the estimation of the time delay because estimation is based on actual data unlike the methods in 7, 8, 9] and [10 where the time delay estimation is based on a range of predicted data.

\subsection{Motivation}

The growing applications of UAVs in both military and civilian sectors require accuracy of system performance and time efficiency of service delivery. One way to achieve this objective is to effectively manage time delays in the system. In nearly all cases, time delays are inevitable chiefly because of considerable distances between the UAVs and the teleoperator and sometimes the number of communication devices input signals must transmit through to get to the UAV. This thesis investigates how to estimate time delays using a bank of recursive least squares filters matched to specific delays. The bank of RLS filters is designed to cover a known range of possible operating time delays in associated with the UAV.

We then look at how time delays can be compensated for by computing an autonomous heading using the estimates obtained from the RLS filter. The simulations are performed on an UAV performing coordinated lateral turns to a set of waypoints. The system is subjected to time delays in heading commands to the waypoint coordinates.

\subsection{Contributions}

1. Bank of recursive least squares filters: We propose to design a bank of RLS filters for a range of time delay and match each RLS filter to a specific time delay. The purpose of the RLS filters is to use the received teleoperator 
commands to estimate a target waypoint and learn the delay.

2. Hypothesis test switch for filter selection: We design a metric from statistical inference with which filters are selected.

3. Autonomous navigation: When the delay and the waypoint are learned, we discussed how delay is compensated for by autonomous navigation. 


\section{Chapter 2}

\section{Aircraft Dynamics and Control}

\subsection{Lateral Control of an Aircraft}

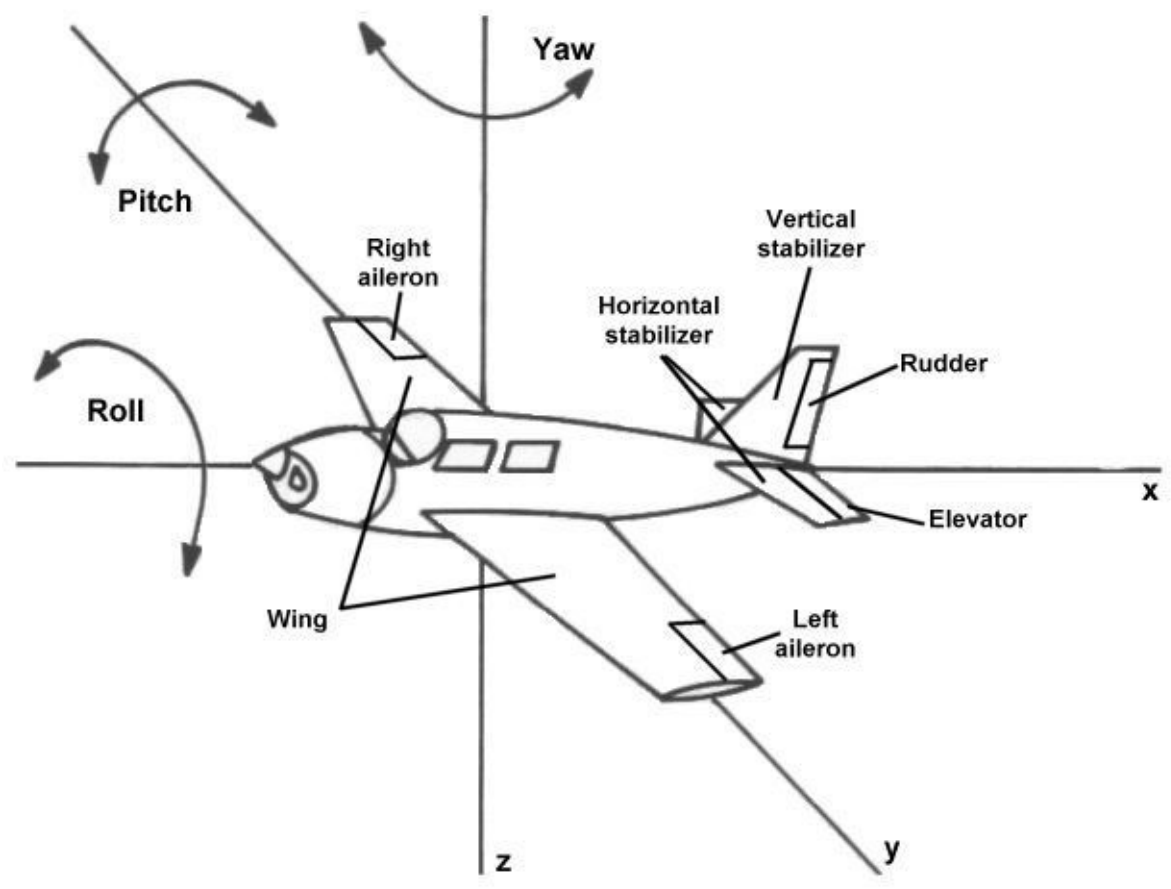

Figure 2.1: Aircraft Control Surface

In this chapter, we design a controller for an aircraft and simulate a coordinated lateral turn. It is expected that when a heading command is given, the aircraft obeys the command and heads in the direction as commanded. The aircraft state 
equations and specifications are obtained from [15. This will serve as the UAV in this thesis. The equation of aircraft dynamics are:

$$
\begin{aligned}
& \dot{\beta}=-0.746 \beta+0.006 p-0.999 r+0.0369 \phi+0.0012 \delta_{A}+0.0092 \delta_{R} \\
& \dot{p}=-12.9 \beta-0.746 p-0.387 r-6.05 \delta_{A}-0.952 \delta_{R} \\
& \dot{r}=4.31 \beta+0.024 p-0.174 r-0416 \delta_{A}-1.76 \delta_{R} \\
& \dot{\phi}=p \\
& \dot{\psi}=r
\end{aligned}
$$

The states are defined as $\beta$ (the side slip angle in rads), $p$ (the the roll rate in rads/sec), $r$ (the yaw rate rads/sec), $\phi$ (the roll angle in rads), $\psi$ (the yaw angle in rads). The inputs to the aileron and rudder are represented as $\delta_{A}$ and $\delta_{R}$ respectively.

When an aircraft makes a turn and the side slip $\beta$ (figure 2.2 is zero, the aircraft then executes a perfect coordinated turn 15. If we equate the first equation of 2.1 to zero, we can derive the expression for the rudder $\delta_{R}$ for which the UAV makes a perfect coordinated turn.

$$
\begin{aligned}
0 & =-0.746 \beta+0.006 p-0.999 r+0.0369 \phi+0.0012 \delta_{A}+0.0092 \delta_{R} \\
\delta_{R} & =-\frac{1}{0.0092}\left(-0.746 \beta+0.006 p-0.999 r+0.0369 \phi+0.0012 \delta_{A}+0.0092 \delta_{R}\right) \\
\delta_{R} & =-0.6562 p+108.59 r-4.011 \phi-81.09 \beta-0.13 \delta_{A}
\end{aligned}
$$

substituting eq. 2.2 in eq. 2.1, we get 


$$
\begin{aligned}
& \dot{\beta}=-81.03 \beta \\
& \dot{p}=-93.99 \beta-1.367 p+103.78 r+3.818 \phi-5.926 \delta_{A} \\
& \dot{r}=-76.78 \beta+1.72 \rho-191.29 r+7.059 \phi-0.187 \delta_{A} \\
& \dot{\phi}=\rho \\
& \dot{\psi}=r
\end{aligned}
$$

Under a perfect coordinated turn, the sideslip $\beta$ is zero. Set $\beta=0$ then we get a third order system with a single control $\delta_{A}$.

$$
\begin{aligned}
& \dot{p}=-1.367 \rho+103.78 r+3.818 \phi-5.926 \delta_{A} \\
& \dot{r}=1.72 \rho-191.29 r+7.059 \phi-0.187 \delta_{A} \\
& \dot{\phi}=\rho \\
& \dot{\psi}=r
\end{aligned}
$$
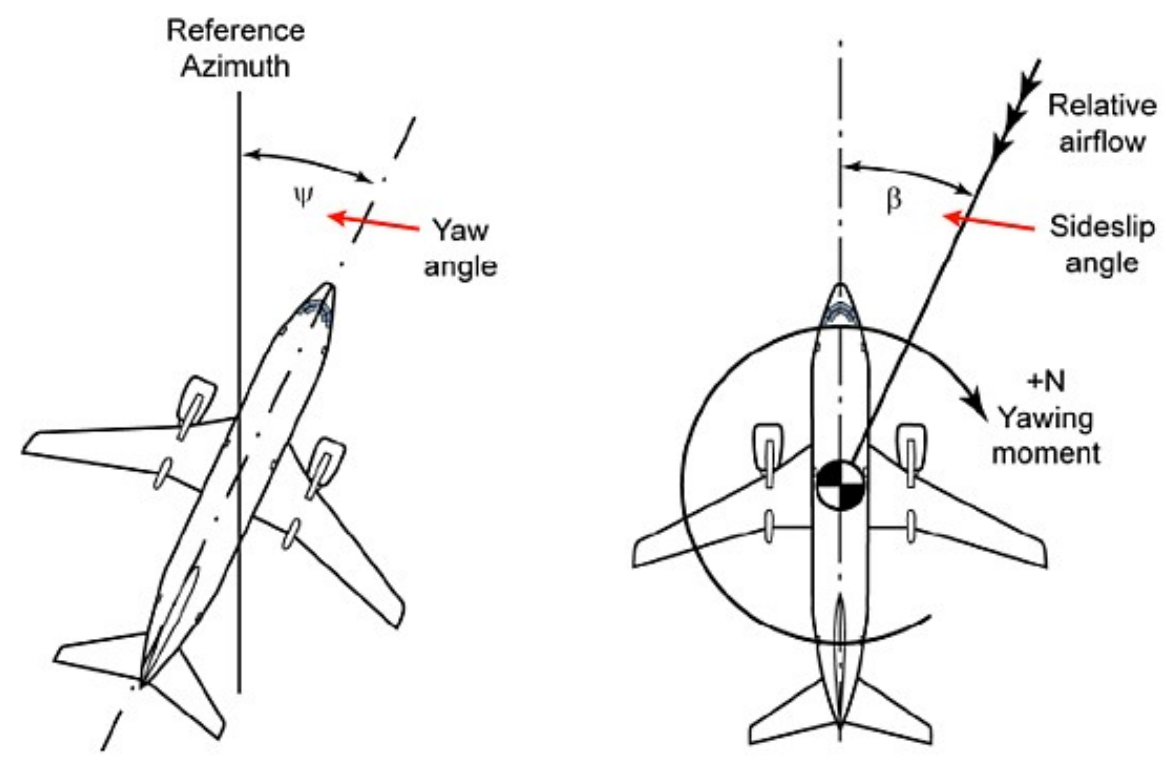

Figure 2.2: Yaw and Sideslip angle 
This system can be represented in state-space as

$$
\begin{aligned}
& \dot{x}=A x+B u \\
& y=C x
\end{aligned}
$$

where,

$$
\begin{aligned}
& A=\left[\begin{array}{cccc}
-1.367 & 103.78 & -3.818 & 0 \\
1.172 & -191.292 & 7.059 & 0 \\
1 & 0 & 0 & 0 \\
0 & 1 & 0 & 0
\end{array}\right] \\
& B=\left[\begin{array}{c}
5.926 \\
-0.187 \\
0 \\
0
\end{array}\right] \\
& C=\left[\begin{array}{llll}
0 & 0 & 0 & 1
\end{array}\right] \\
& x=\left[\begin{array}{llll}
p & r & \phi & \psi
\end{array}\right]^{T} \\
& u=\delta_{A}
\end{aligned}
$$

The states are defined as roll rate $\mathrm{p} \mathrm{rad} / \mathrm{s}$, yaw rate $\mathrm{r} \mathrm{rad} / \mathrm{s}$, roll angle $\phi \mathrm{rad}$ and yaw angle $\psi$ rad. 


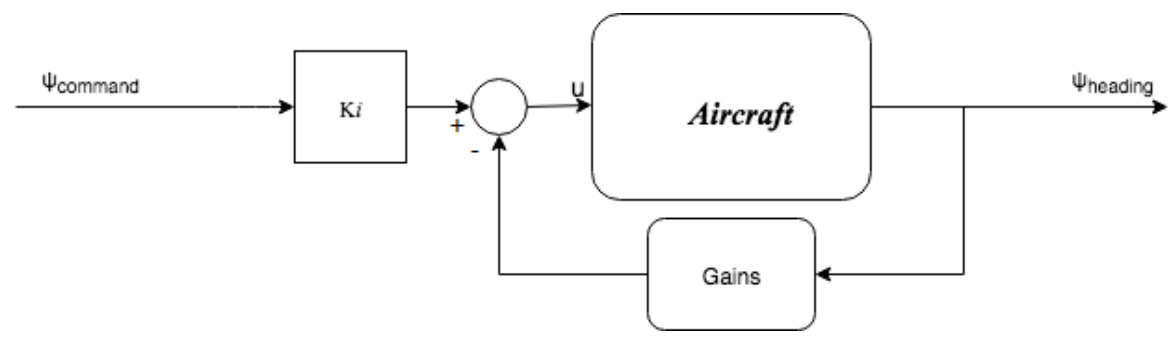

Figure 2.3: Aircraft system

The reference state is the heading command which indicates the lateral turn angle of the aircraft (i.e the yaw angle, $\psi$ ). $K_{i}$ is the gain of the reference state, the yaw angle $\psi$.

\subsubsection{Pole Placement}

Using the MATLAB eig(A) command line we obtain the eigenvalues of the system. The eigenvalues are:

$$
\left[\begin{array}{llll}
0 & -191.9102 & 0.0152 & -0.7640
\end{array}\right]
$$

Two important things to note from the eigenvalues. The zero indicates there is a free integrator. One can consider this a third order system augmented with the integral state of yaw angle see figure 2.2. The yaw angle is already the integral component. The system is unstable, and the aircraft will crash without some type of feedback. The unstable root is at $s=0.0152$.

We are assuming full state feedback and we can do pole placement arbitrarily. In 15 the author suggests that poles should be placed at $-1,-1 \pm 3 j$. Placing the poles as suggested produced large feedback gains:

$$
\text { Gains }=\left[\begin{array}{llll}
-5.0415 & 848.1360 & -31.2855 & 0.2432
\end{array}\right]
$$




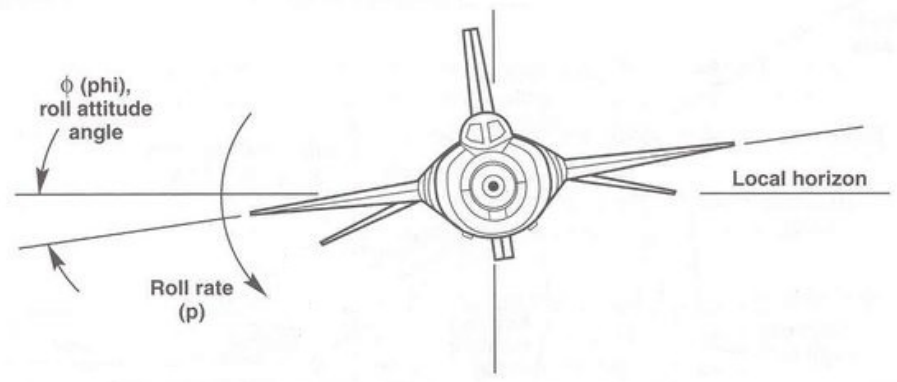

Figure 2.4: Roll angle

The gains are multiplied with the system's state vector $[\rho, r, \phi, \psi]$ which produce large state values and the large gains will cause the control surfaces to saturate (see figure 2.5). Note that in this academic scenario, an aileron deflection of 7 radians $\left(401.02^{\circ}\right)$ is not possible. The maximum allowable aileron deflection in an aircraft should be between 1.047 radians $\left(60^{\circ}\right)$ to 1.31 radians $\left(75^{\circ}\right)$. Furthermore a 13 radians roll angle would mean that the aircraft rolls several times over, see figure 2.4 

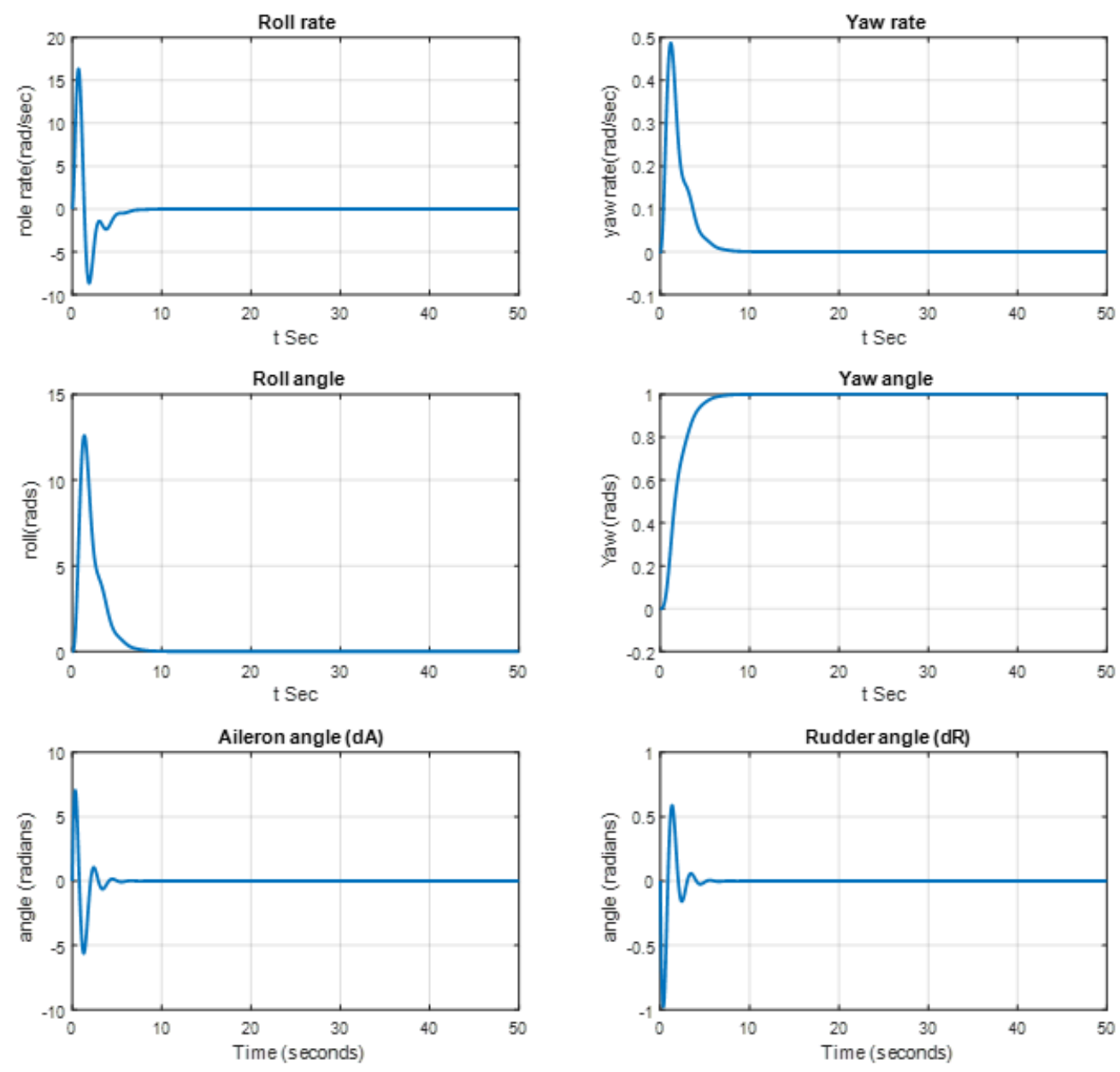

Figure 2.5: Unit step response of $\rho, \mathrm{r}, \phi, \psi$ with poles placed at $-1,-1 \pm 3 j$ as suggested in 15. The max aileron angle $\delta_{A}$ is about $401.02^{\circ}(7.01 \mathrm{rad})$.

We did a design iteration to keep the roll angle at a reasonable degree such that the aircraft does not roll over. The closed loop poles were chosen as:

$$
J=\left[\begin{array}{llll}
-0.1 & -0.1-0.1 i & -0.1+0.1 i & -191.9102
\end{array}\right]
$$

The dominant poles are at $-0.1 \pm 0.1 j$ with a damping ratio $\zeta=0.707$ and natural 
frequency $\omega_{n}=0.1414$ and a settling time of approximately 40 seconds. The resulting system behaviour is shown in figure 2.10. Using the MATLAB acker function 16 for pole placement,

$$
K=\operatorname{acker}(A, B, J)
$$

the gains are

$$
K=\left[\begin{array}{llll}
-0.0771 & -0.0418 & 0.0104 & 0.0093
\end{array}\right]
$$

The closed loop system dynamics are given by

$$
\begin{aligned}
\dot{x} & =\hat{A} x+\hat{B} \psi_{\text {command }} \\
& =(A-B K) x+B k_{4} \psi_{\text {command }} \\
\hat{A} & =(A-B K) \\
\hat{A} & =\left[\begin{array}{cccc}
-0.9104 & 104.0276 & -3.8795 & -0.0553 \\
1.1576 & -191.2998 & 7.0609 & 0.0017 \\
1.0000 & 0 & 0 & 0 \\
0 & 1.0000 & 0 & 0
\end{array}\right] \\
\hat{B} & =B k_{4} \\
& =B \times 0.0093
\end{aligned}
$$

once again taking the eigenvalues we get

$$
\left[\begin{array}{llll}
-191.91 & -0.1+0.1 i & -0.1-0.1 i & -0.1
\end{array}\right]
$$




\subsubsection{Controller Design}

All the poles are placed on the left half of the imaginary plane in specified pole positions, and as such this is a stable system. The system is simulated in MATLAB, the input to the aileron is the feedback

$$
\delta_{A}=-K x+0.0093 \psi_{\text {command }}
$$

where, $\psi_{\text {command }}$ is the heading command. The heading command $\psi_{\text {command }}$ is multiplied by the gain $\mathrm{K} \psi$ to match the feedback control gain.

In MATLAB,

$$
S Y S=\operatorname{ss}(\hat{A}, \hat{B}, C, D)
$$

will create a continuous time state-space model of the aircraft system. Then using the MATLAB step function the unit-step response is obtained.

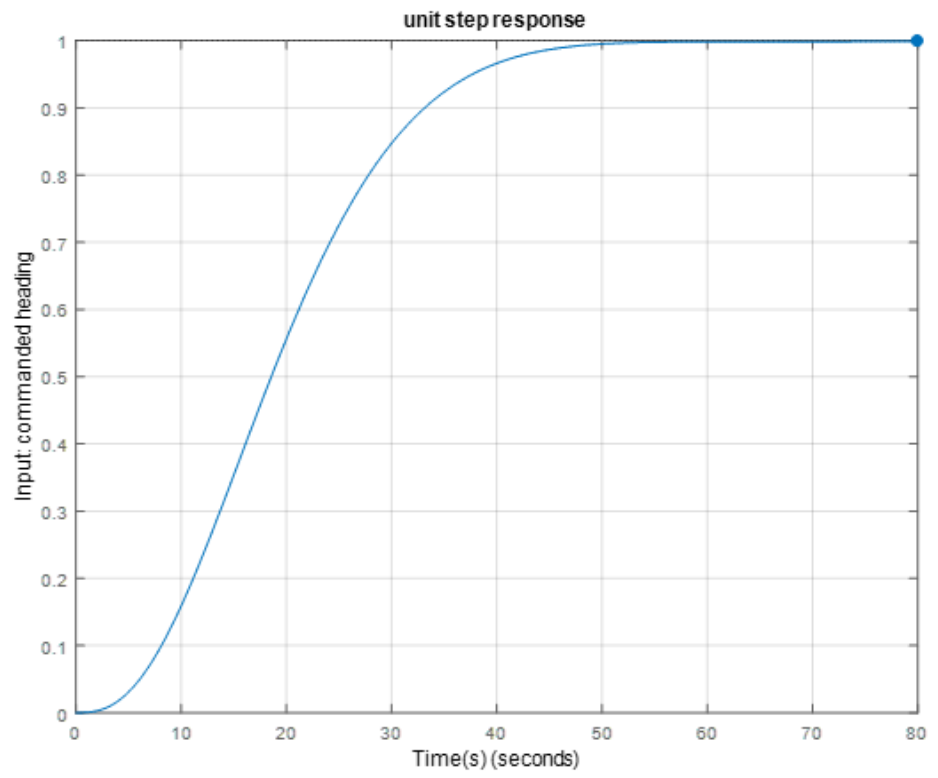

Figure 2.6: Unit step response 
If the system receives an input heading command, the system should head in direction as commanded. Figures 2.6, 2.7 and 2.8 show the controlled behaviour with $30^{\circ}, 45^{\circ}$ and $60^{\circ}$ heading commands.

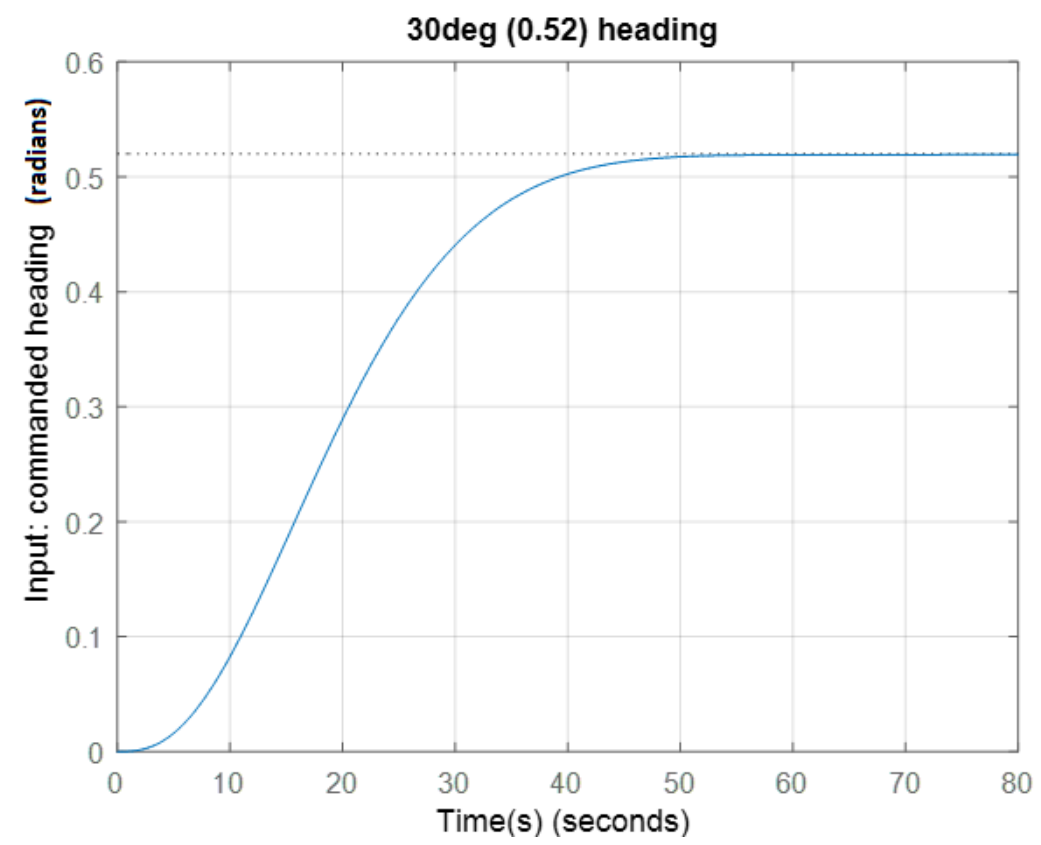

Figure 2.7: $30^{\circ}(0.52 \mathrm{rad})$ response

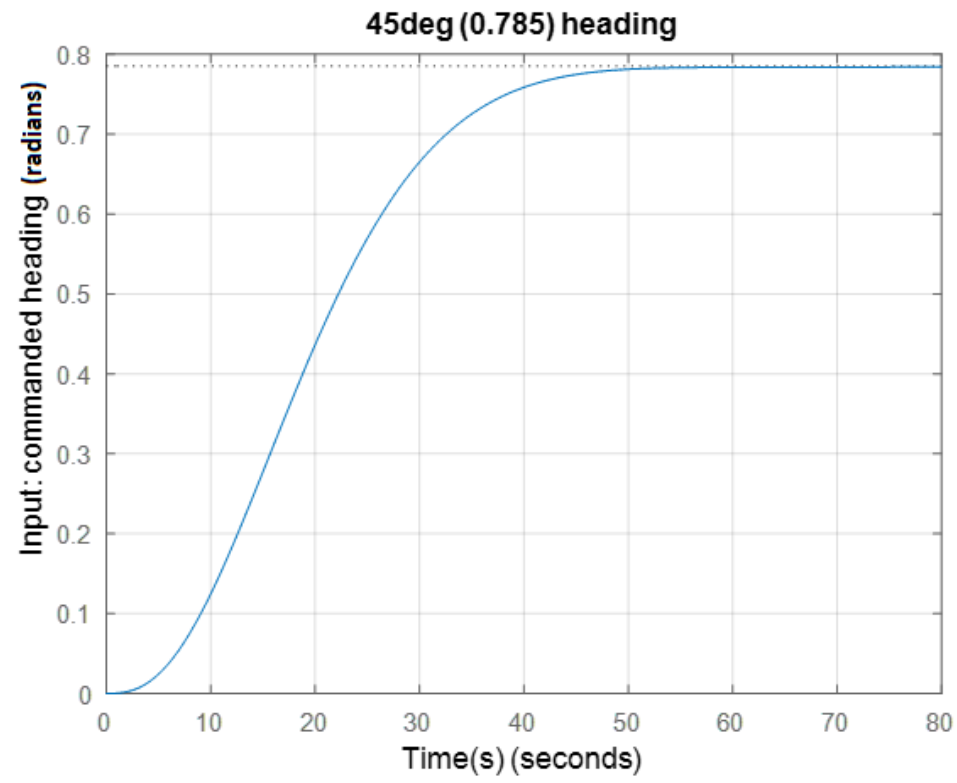

Figure 2.8: $45^{\circ}(0.785 \mathrm{rad})$ response 




Figure 2.9: $60^{\circ}$ (1.047 rad) response

The response of the states $[\rho, r, \phi, \psi]$ is shown below. 

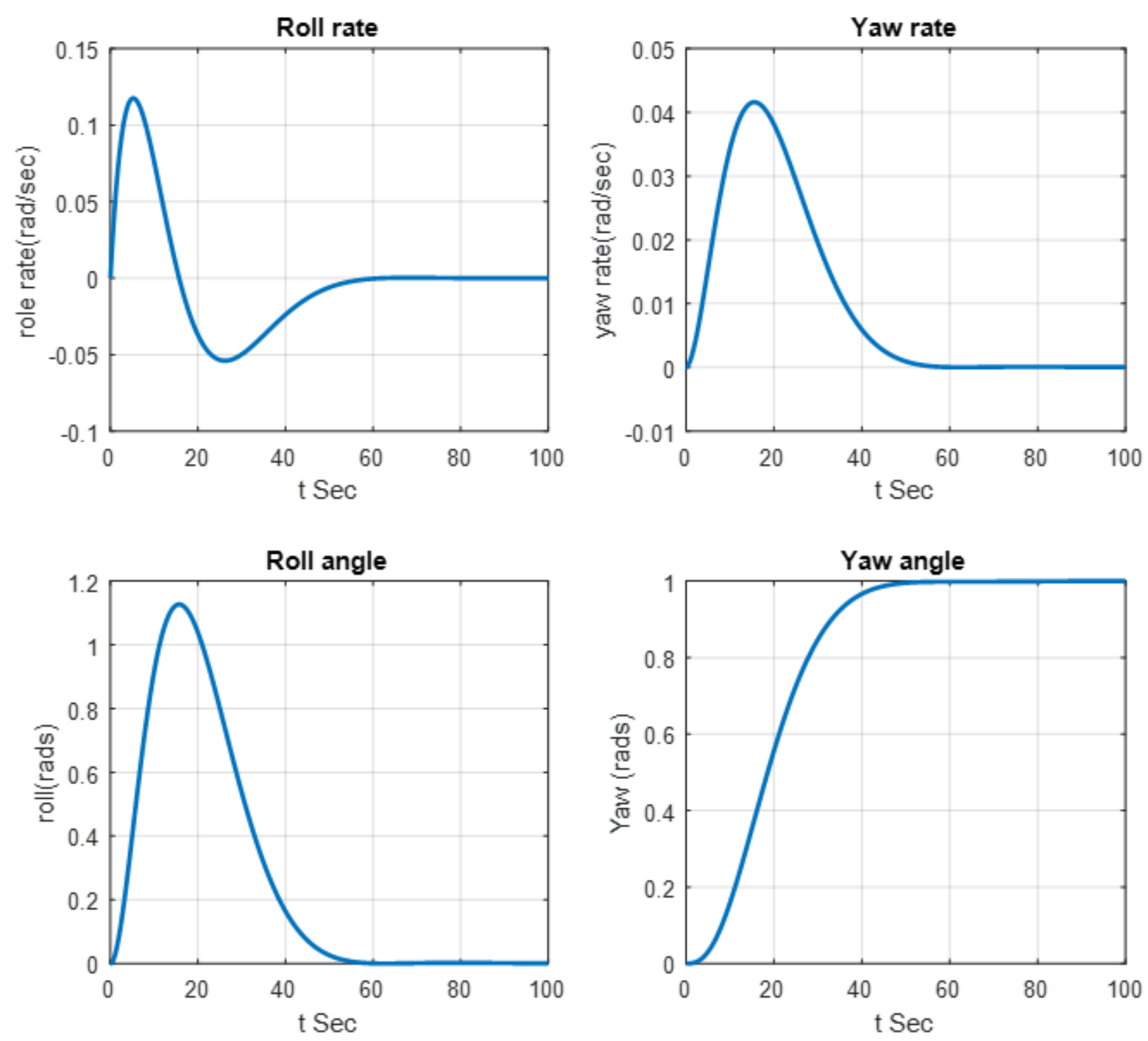

Figure 2.10: Unit step response of $\rho, \mathrm{r}, \phi, \psi$.

The rudder angle defined in eq. 2.2 is plotted in figure 2.11 


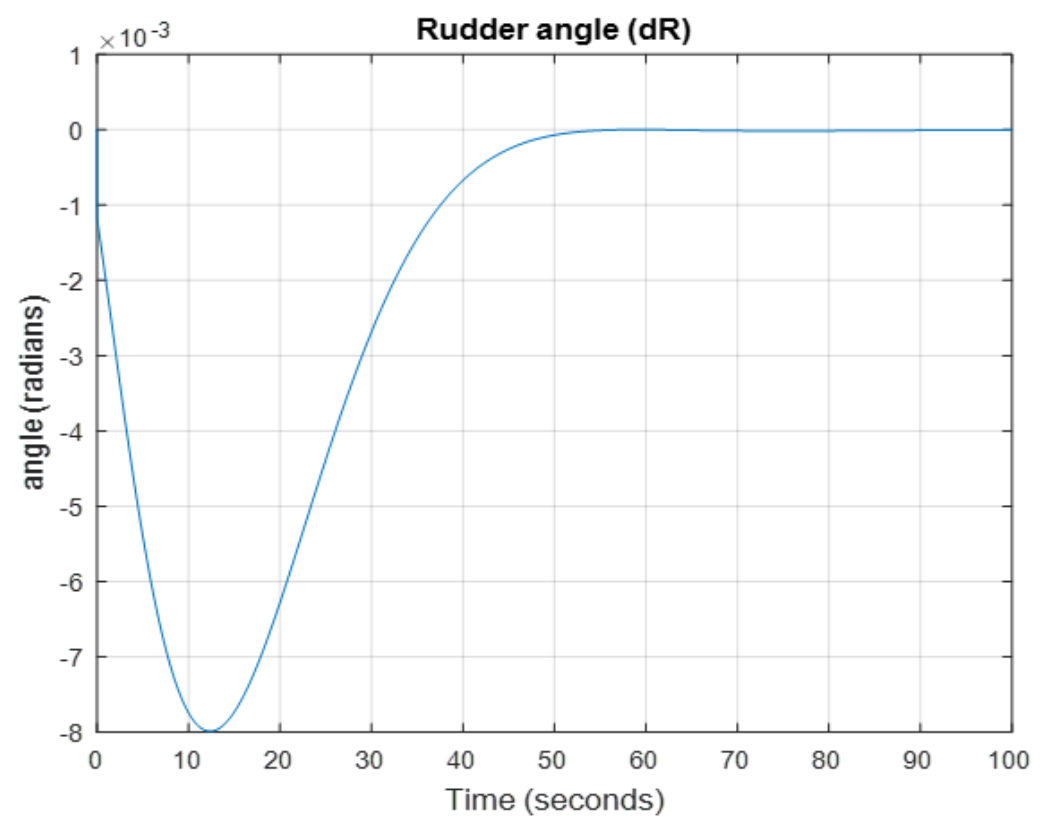

Figure 2.11: Rudder angle.

The Rudder rotates with a maximum deflection angle of $0.46^{\circ}$. The aileron angle $\delta_{A}$ as given by eq 2.18 is

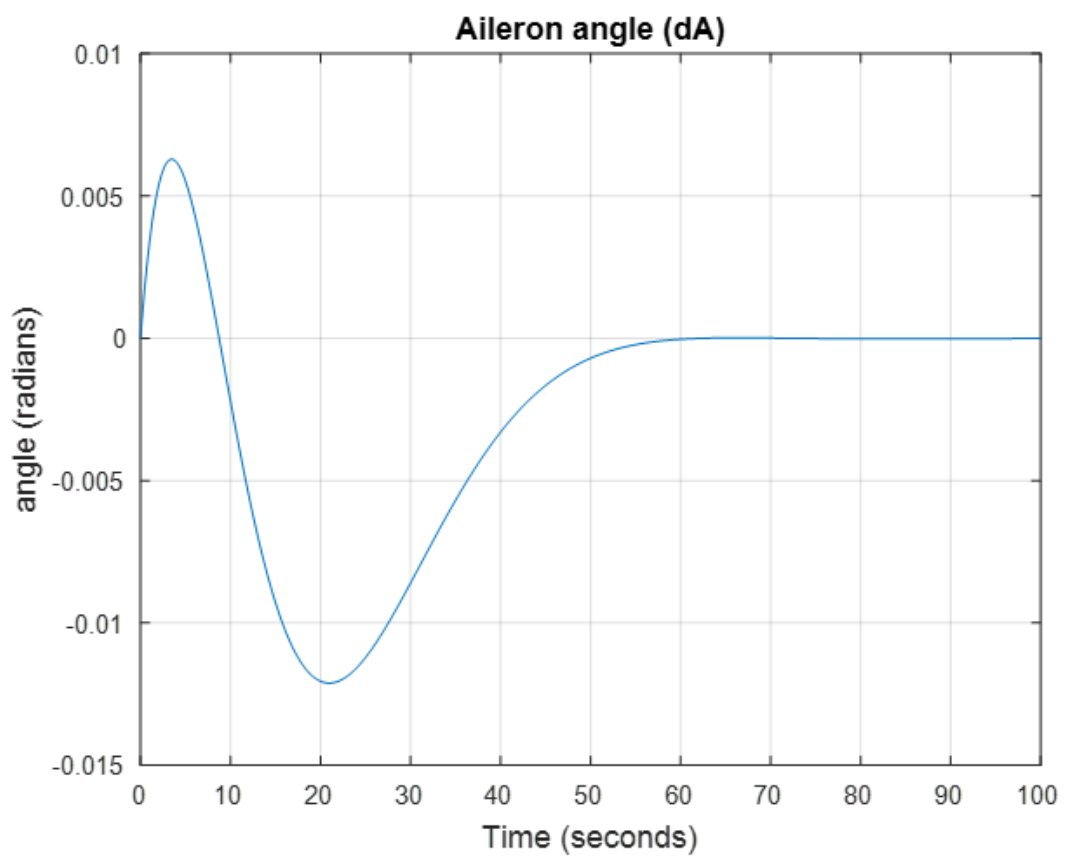

Figure 2.12: Aileron angle. 
The maximum deflection angle of the aileron is $0.74^{\circ}$. One can see that the control surfaces do not saturate as discussed in 2.1.1

The aircraft's trajectory on the $x-y$ plane is illustrated in figures 2.13 and 2.14 . The aircraft system is simulated using the MATLAB's ode 45 function, which is a runge-kutta integrator. Two states $x$ and $y$ are added to give the aircraft cartesian coordinates.

$$
\begin{aligned}
& \dot{x}=v \cos \psi \\
& \dot{y}=v \sin \psi
\end{aligned}
$$

where $v$ is constant velocity. For this simulation, $v=55.556 \mathrm{~m} / \mathrm{s},(200 \mathrm{~km} / \mathrm{hr})$. We input two heading commands $\psi_{\text {command }}=30^{\circ}$ and $\psi_{\text {command }}=45^{\circ}$, to test the performance of the controller.

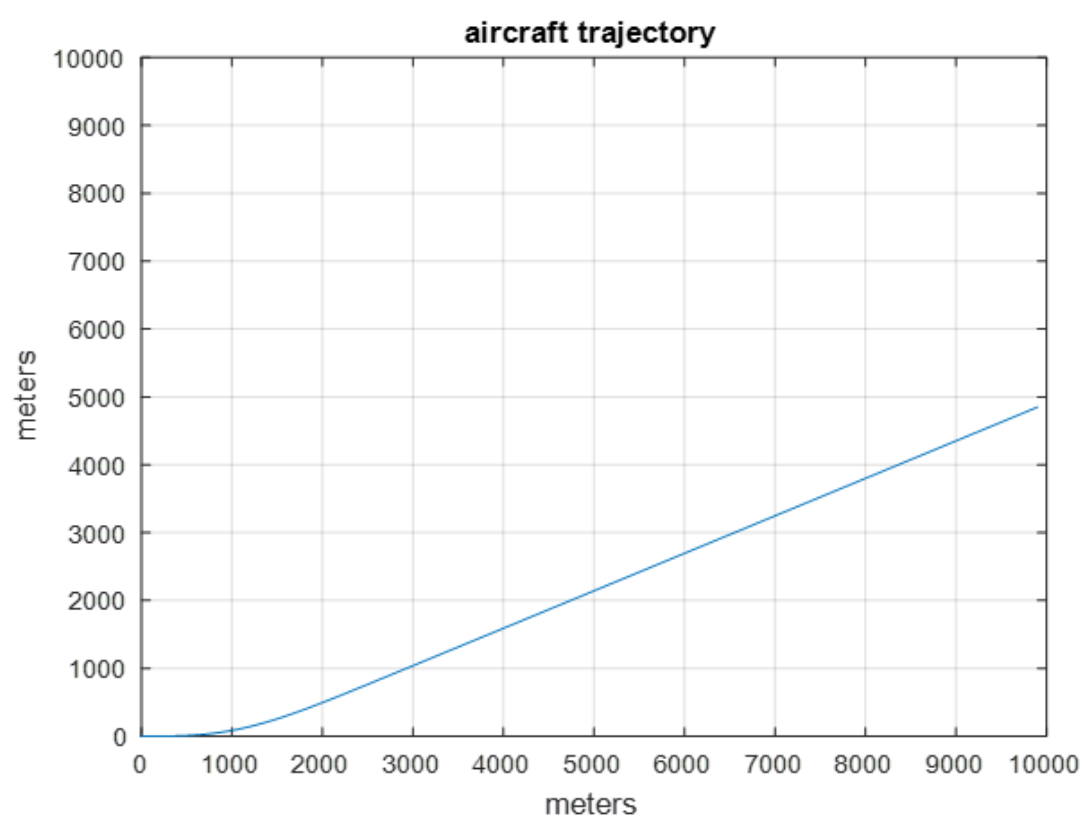

Figure 2.13: Aircraft trajectory subjected to a $30^{\circ}$ heading command. 


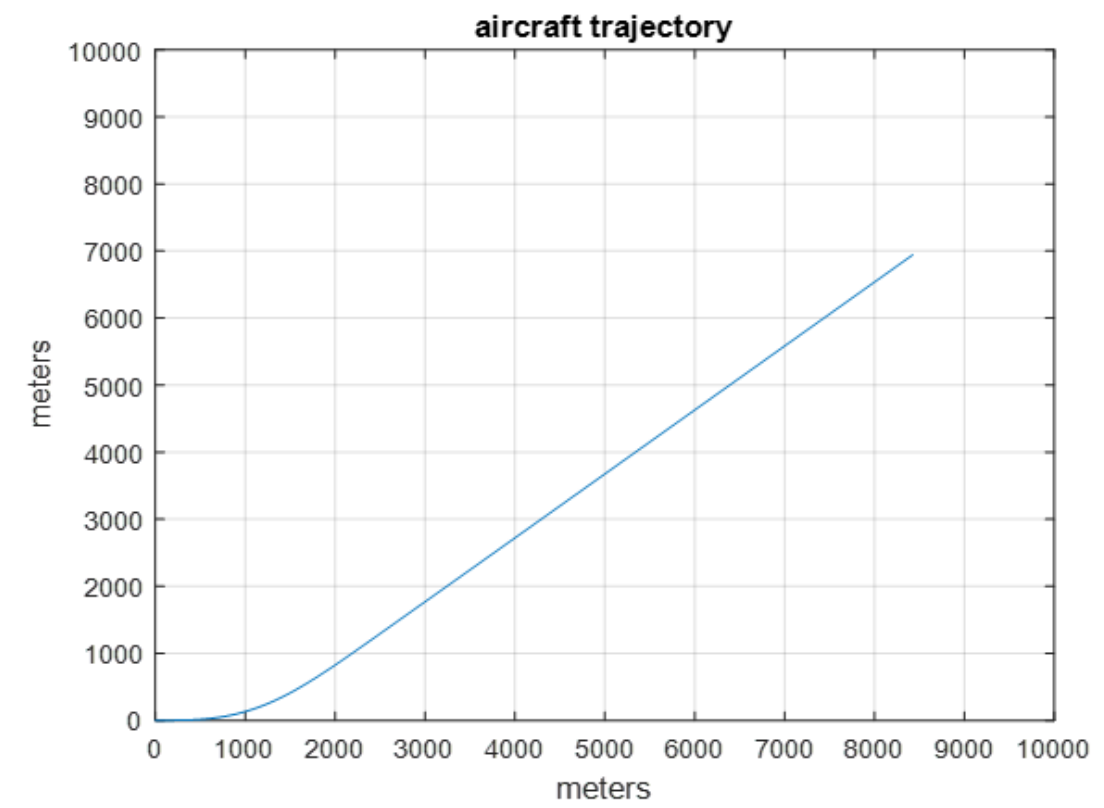

Figure 2.14: Aircraft trajectory subjected to a $45^{\circ}$ heading command.

In figure 2.15, the aircraft is simulated as it is being teleoperated to make coordinated lateral turns to different waypoints. We set up the waypoint coordinates as

$$
\begin{aligned}
& x_{\text {waypoint }}=\left[\begin{array}{lllllll}
0 & 2000 m & 7000 m & 15000 m & 18000 m & 12000 m & 2000 m
\end{array}\right] \\
& y_{\text {waypoint }}=\left[\begin{array}{lllllll}
0 & 5000 m & 8000 m & 8000 m & 4000 m & 2000 m & 10000 m
\end{array}\right]
\end{aligned}
$$

The heading command is defined as

$$
\psi_{\text {command }}(t)=\tan ^{-1}\left(\frac{y_{\text {waypoint }}-y(t)}{x_{\text {waypoint }}-x(t)}\right)
$$


where,

$$
\begin{aligned}
\psi_{\text {command }}(t) & =\text { the heading command at time } \mathrm{t} . \\
x(t), y(t) & =\text { the aircraft cartesian coordinates at time } \mathrm{t} .
\end{aligned}
$$

Plot of the aircraft trajectory to the waypoints as shown below.

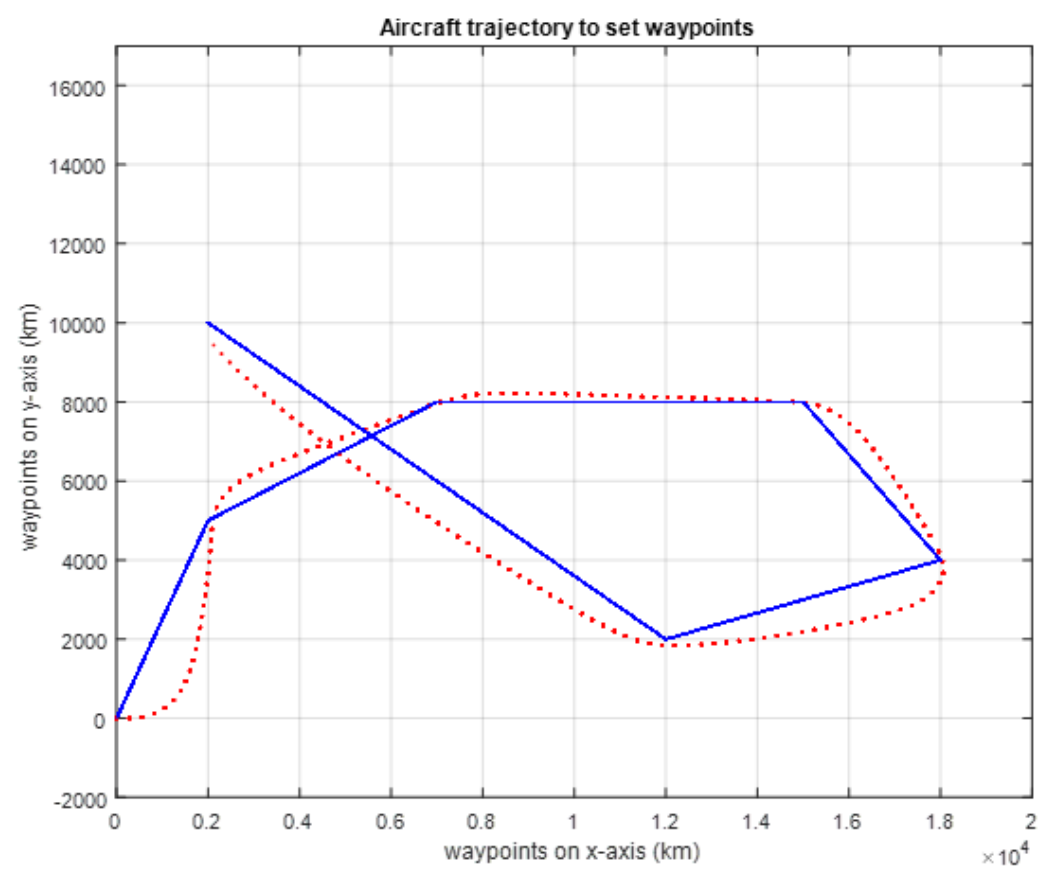

Figure 2.15: Aircraft trajectory. The red dotted line indicates the aircraft trajectory and the blue line is the straight line between waypoints labelled $A$ to $F$.

The teleoperator instantaneously commands the UAV according to $\psi_{\text {command }}$ as given by eq 2.20. The UAV is travelling at a constant velocity and when the UAV gets within a threshold distance of the waypoint, then the operator selects a heading to the next waypoint. The threshold distance is a radius within which the UAV is said to have reached the target waypoint. The threshold is user defined based on the application. 


\subsection{Time Delays}

In chapter one we briefly summarized the effects of time delays on UAVs. In this section, the aircraft is simulated as it receives delayed input commands. In figure 2.16, we see the unit step response of a 40 seconds delayed input. It can be observed that the response of both the delayed input command and input with no-delay are the same, however, the delayed response takes longer time $(t+$ time delay $)$ to reach steady state. This is an undesirable system behaviour especially in time sensitive UAV applications.

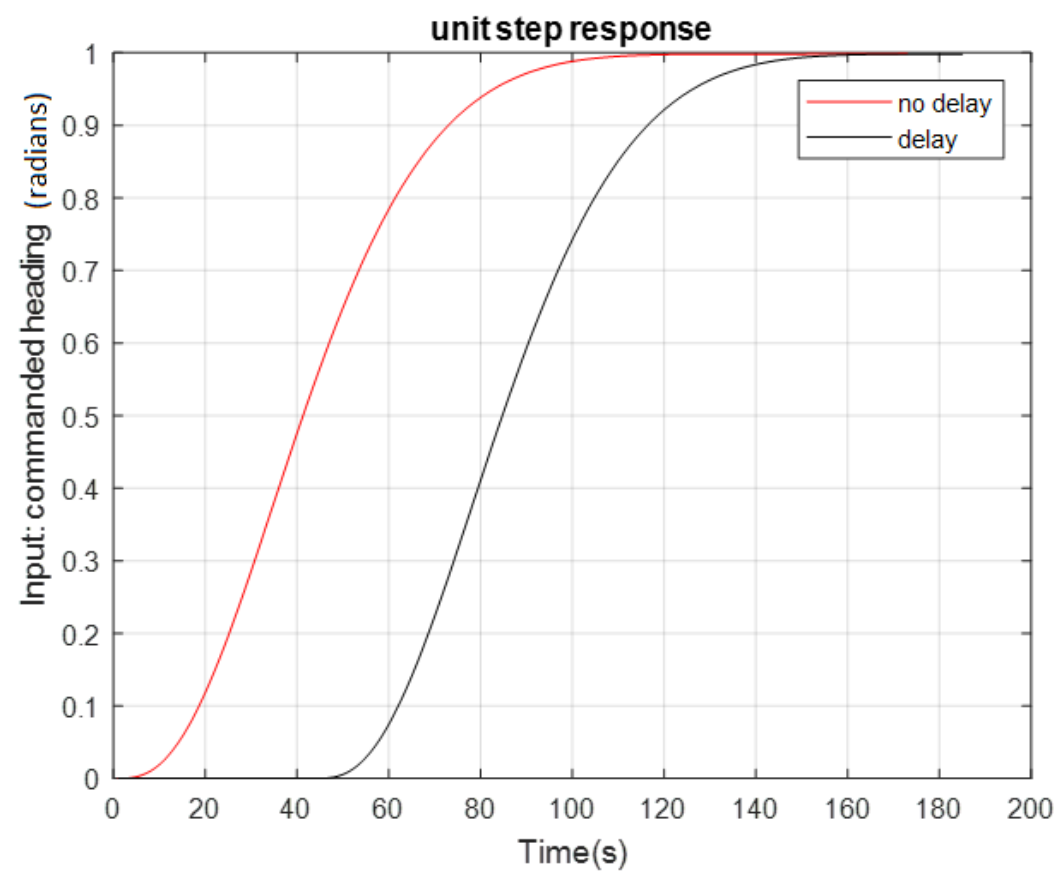

Figure 2.16: Unit step response with a delayed input command.

Figures 2.17 to 2.21 illustrate the trajectory of the UAV in section 2.1 under the effects of the different time delays as it moves to the set waypoints. Wind noise $N_{w}$ is simulated as a gaussian noise coming from the north east at $45^{\circ}$ angle $(0.786$ 
radians). Eq 2.19 is modified to:

$$
\begin{gathered}
\dot{x}=v \cos \psi+N_{w} \cos (0.786) \\
\dot{y}=v \sin \psi-N_{w} \sin (0.786) \\
N_{w}=v_{w}(1+0.1 \sin \omega t)
\end{gathered}
$$

where $N_{w}$ is the sinusoidal wind noise with velocity $v_{w}=13.89 \mathrm{~m} / \mathrm{s}$, an amplitude of 0.1 , angular frequency $\omega=0.1 \mathrm{rad} / \mathrm{s}$ and $t=1 \mathrm{~s}$.

The aircraft is simulated with time delays of $2 \mathrm{~s}, 5 \mathrm{~s}, 10 \mathrm{~s}, 20 \mathrm{~s}$, and $30 \mathrm{~s}$ in the input command. When a heading command is sent at time $t$, it is sent based on the UAV's coordinate $\left(x_{\text {uav }}(t), y_{\text {uav }}(t)\right)$. The UAV receives the heading command at time $(t+$ time delay $)$ at a new coordinate $\left(x_{\text {uav }}(t+\right.$ time delay $), y_{\text {uav }}(t+$ time delay $\left.)\right)$. The UAV continues to head in the direction of the last received command for the period of the active time delay until it receives a new command.

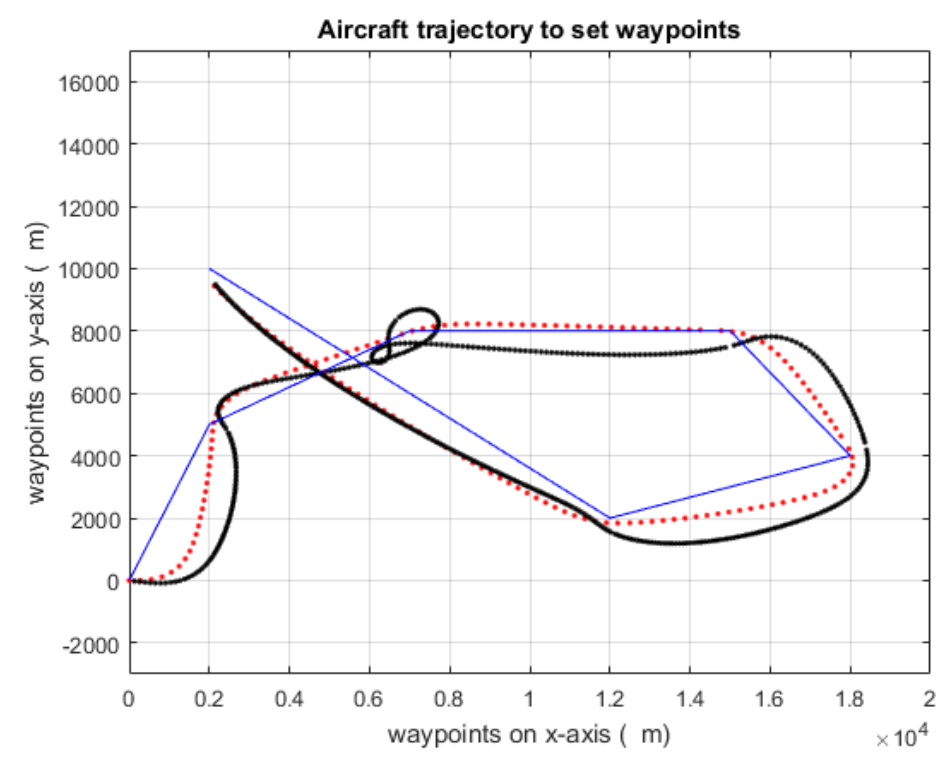

Figure 2.17: Trajectory with 2 seconds delay. The red dotted line indicates the aircraft trajectory with no delay, the blue line is the straight line between waypoints and the black line indicates the UAV trajectory subjected to time delay 


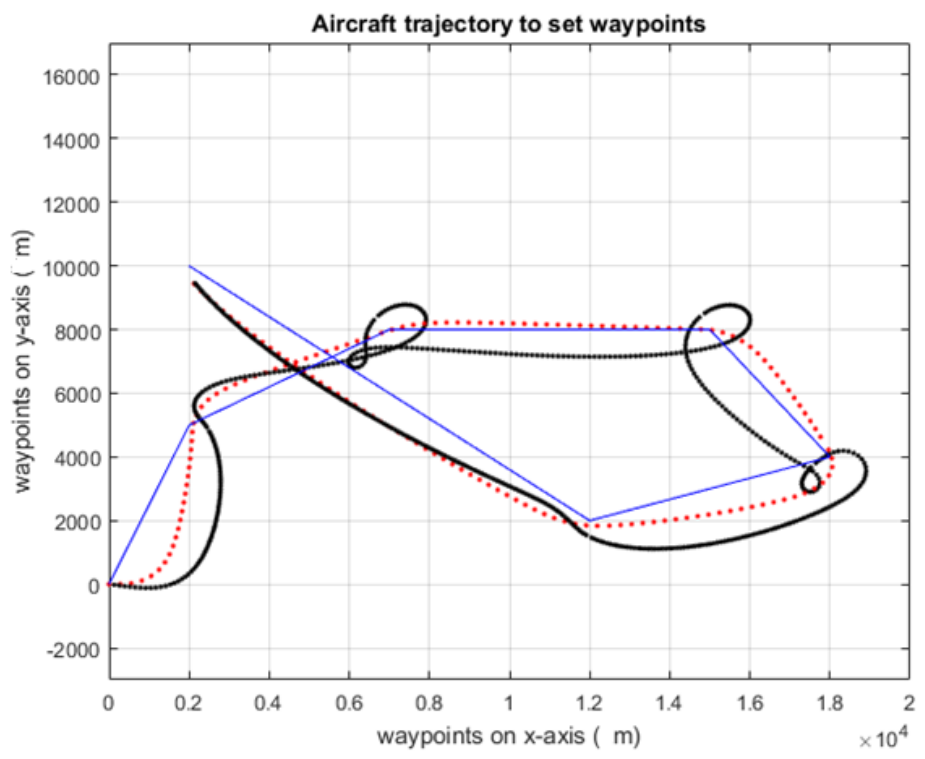

Figure 2.18: Trajectory with 5 seconds delay. The red dotted line indicates the aircraft trajectory with no delay, the blue line is the straight line between waypoints and the black line indicates the UAV trajectory subjected to time delay 


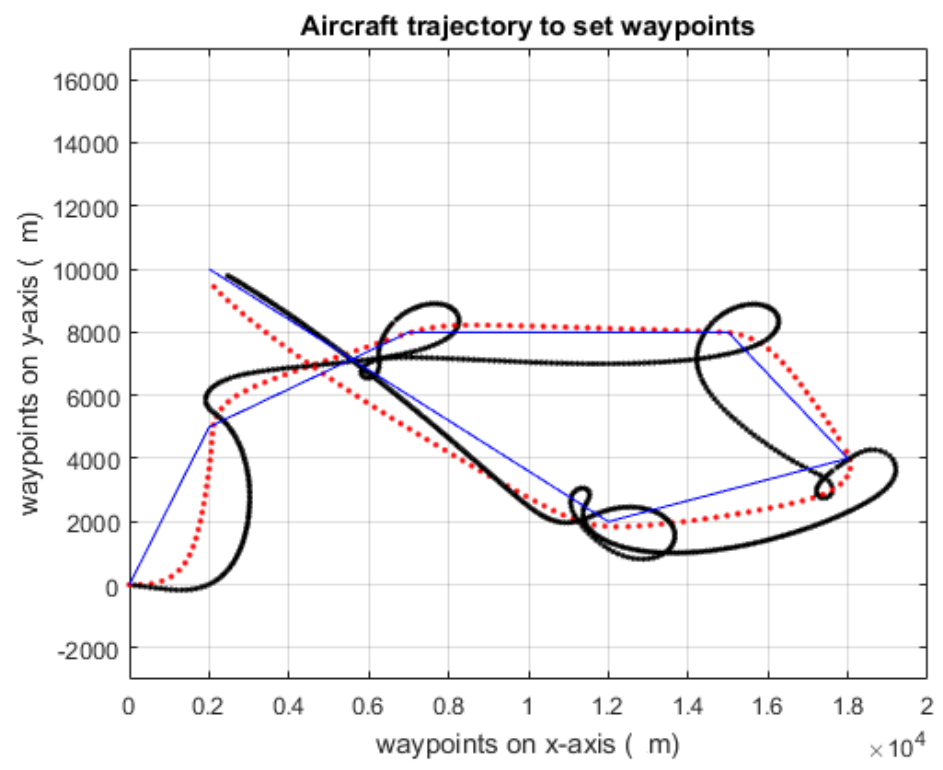

Figure 2.19: Trajectory with 10 seconds delay. The red dotted line indicates the aircraft trajectory with no delay, the blue line is the straight line between waypoints and the black line indicates the UAV trajectory subjected to time delay

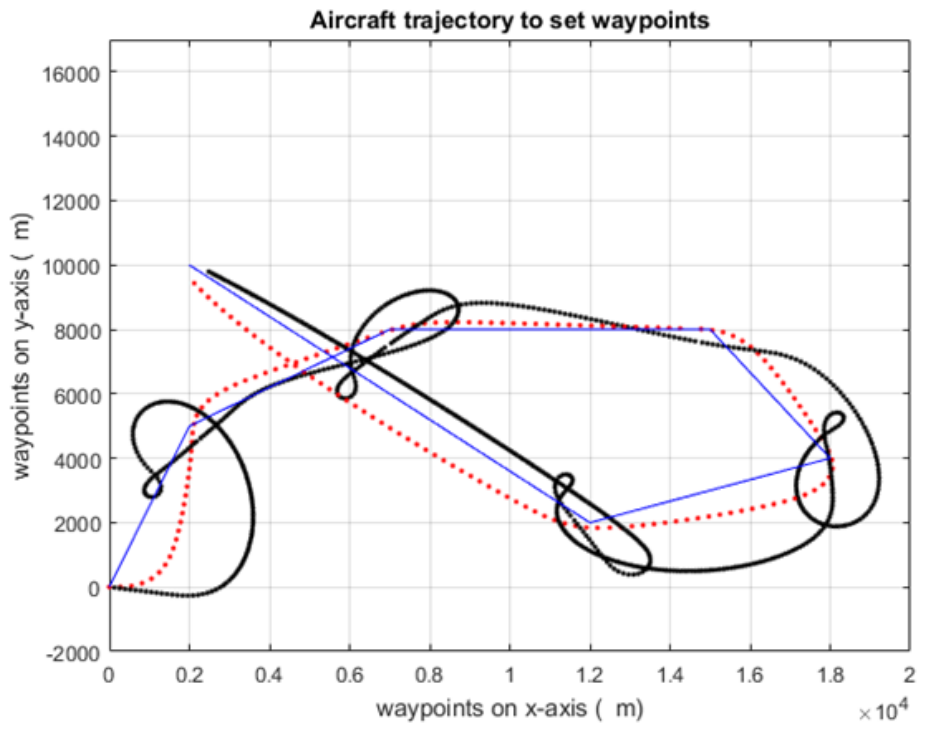

Figure 2.20: Trajectory with 20 seconds delay. The red dotted line indicates the aircraft trajectory with no delay, the blue line is the straight line between waypoints and the black line indicates the UAV trajectory subjected to time delay 


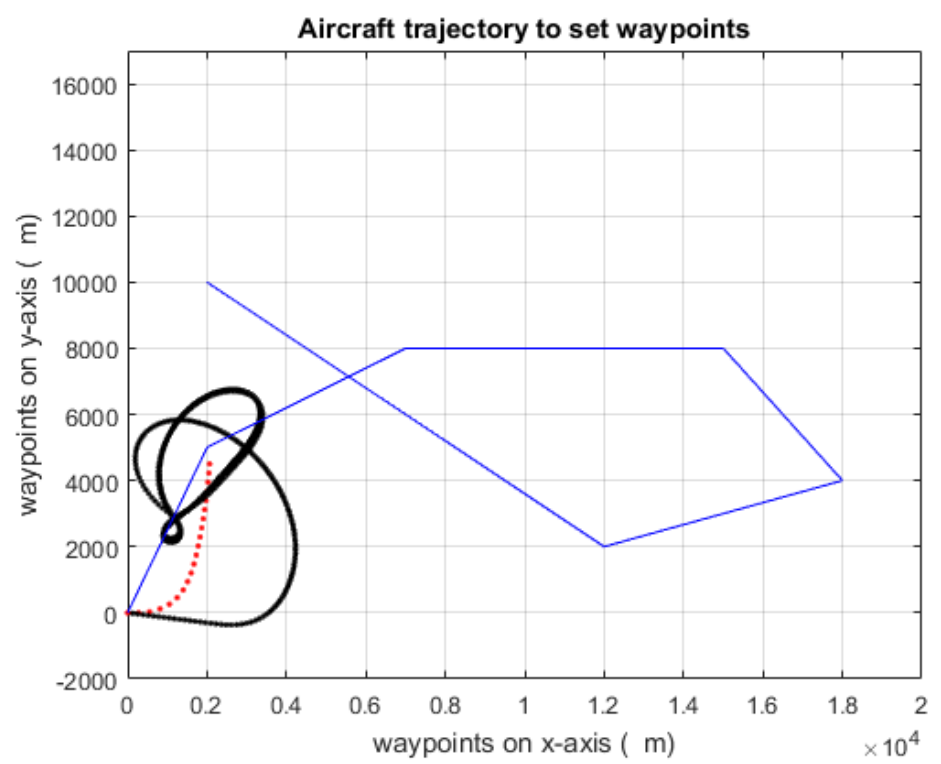

Figure 2.21: Trajectory with 30 seconds delay. The red dotted line indicates the aircraft trajectory with no delay, the blue line is the straight line between waypoints and the black line indicates the UAV trajectory subjected to time delay

From the figures it can be observed that there is a larger displacement between the trajectory of UAV with delayed commands (black) and the straight line between the waypoint(blue) compared to the UAV trajectory without delay (red). We can say that when the input commands are subjected to time delay, the UAV travels longer distances and consequently, travel time is increased. We also see that the UAV misses target waypoints and then circles back in an attempt to reach the way point again, spending even more time in the process. In military attack applications or medical life-saving applications, where reaching a target location within an exact time frame can not be compromised, this is unacceptable. The UAV subjected to a 30 seconds time delay figure 2.21, keeps missing the waypoint and even when it circles back to attempt reaching the target, it misses it again. As seen in figure 2.21, the operator loses control of the UAV and the UAV keeps going in circles.

In the next chapter we proposed a method for which the UAV can identify the delays and then compensate for the delays. 


\section{Chapter 3}

\section{Compensation for Delay}

\subsection{Brief Review of Least Squares(LS) and Re- cursive Least Square(RLS) Estimation}

\subsubsection{Least Squares(LS) Estimate}

The least squares algorithm is a well-known algorithm for fitting experimental data to a model [17. The first step is to define a mathematical model that is believed to fit the data and then design an experiment to obtain the data. For example, let us look at a two-dimensional linear regression fit:

$$
y(n)=a x(n)+b
$$

where $x(n)$ is the input signal and $y(n)$ is the output. This is analogous to the equation of a straight line. It is assumed that a straight line model will fit the data. Equation 3.1 can be written in the form

$$
y(n)=\phi^{T} \theta
$$


where $\phi^{T}=[x(n) 1]$ and $\theta^{T}=\left[\begin{array}{ll}a & b\end{array}\right]$. The objective of Least Squares is to find the optimal parameter estimate $\hat{\theta}$ that minimizes the cost function

$$
J=\frac{1}{2} \frac{1}{N} \sum_{n=1}^{N}\left(y(n)-\phi^{T}(n) \hat{\theta}\right)^{2}
$$

The term $\hat{\theta}$ is the Least Squares estimate of the parameter $\theta$. To find $\hat{\theta}$, we take the partial derivative of the cost function $J$ with respect to $\hat{\theta}$ and set this partial derivative to zero. Therefore, we get

$$
\begin{aligned}
\frac{\partial J}{\partial \hat{\theta}} & =\frac{1}{N} \sum_{n=1}^{N}\left(y(n)-\phi^{T}(n) \hat{\theta}\right) \phi(n) \\
& =\frac{1}{N} \sum_{n=1}^{N}\left(\phi(n) y(n)-\frac{1}{N} \sum_{n=1}^{N} \phi(n) \phi^{T}(n) \hat{\theta}\right)
\end{aligned}
$$

Setting $\frac{\partial J}{\partial \hat{\theta}}=0$, we get

$$
\sum_{n=1}^{N}\left(\phi(n) y(n)=\sum_{n=1}^{N} \phi(n) \phi^{T}(n) \hat{\theta}\right)
$$

therefore,

$$
\hat{\theta}=\left[\sum_{n=1}^{N} \phi(n) \phi^{T}(n)\right]^{-1}\left[\sum_{n=1}^{N} \phi(n) y(n)\right]
$$

Where the inverse $\left[\sum_{n=1}^{N} \phi(n) \phi^{T}(n)\right]^{-1}$ exists, if it does not exist the system can not be identified. The matrix $\left[\sum_{n=1}^{N} \phi(n) \phi^{T}(n)\right]$ is the information matrix I. The matrix $\left[\sum_{n=1}^{N} \phi(n) \phi^{T}(n)\right]^{-1}$ is the covariance matrix P.

Equation (3.4) can written in the form

$$
\frac{\partial J}{\partial \hat{\theta}}=0=\frac{1}{N} \sum_{n=1}^{N}\left(y(n)-\phi^{T}(n) \hat{\theta}\right) \phi(n)
$$


and we can define the prediction errors as

$$
\epsilon(n)=\left(y(n)-\phi^{T}(n) \hat{\theta}\right) .
$$

$\epsilon(n)$ represents the errors in predicting the output of the system. The linear regression is typically written as

$$
y(n)=\phi^{T}(n)+v(n)
$$

where $v(n)$ represents the modelling noise. Let us consider a second-order system whose model is defined by

$$
y(n)=-a_{1} y(n-1)-a_{2} y(n-2)+b_{1} u(n-1)+b_{2} u(n-2)+v(n)
$$

The model structure is defined in $\phi(n)$ as

$$
\phi^{T}(n)=\left[\begin{array}{llll}
y(n-1) & y(n-2) & u(n-1) & u(n-2)
\end{array}\right]
$$

Generally, we can represent an arbitrary $k$ th-order autoregressive exogenous (ARX) 17 model structure as

$$
\begin{aligned}
& y(n)=-a_{1} y(n-1)-a_{2} y(n-2)-\ldots-a_{m} y(n-k) \\
& +b_{1} u(n-1)+b_{2} u(n-2)+\ldots+b_{n-k} u(n-k)+v(n)
\end{aligned}
$$

$\phi^{T}(n)$ is represented by

$$
\phi^{T}(n)=\left[\begin{array}{llllll}
y(n-1) & \ldots & y(n-k) & u(n-1) & \ldots & u(n-k)
\end{array}\right]
$$


The LS can be written in matrix form as

$$
\Phi=\left[\begin{array}{llll}
\phi(1) & \phi(2) & \ldots & \phi(N)
\end{array}\right]
$$

and the output matrix as

$$
Y=\left[\begin{array}{llll}
y(1) & y(2) & \ldots & y(N) .
\end{array}\right]
$$

The LS estimate is written as

$$
\hat{\Theta}=\left(\Phi \Phi^{T}\right)^{-1} \Phi Y
$$

And the prediction errors is written in the form

$$
E=Y-\Phi^{T} \hat{\Theta}
$$

\subsubsection{Recursive Least Squares(RLS) Estimate}

In the LS algorithm, we first had to collect $\mathrm{N}$-data then get the LS estimates from Eq. 3.6. The RLS algorithm is used to obtain parameter estimates in real time. The RLS algorithm is derived by assuming a solution to the LS algorithm and then adding a single data point. Recall from Eq. 3.6. , the conventional LS solution based on $N$ data points is given as:

$$
\hat{\theta}_{L S}^{N}=\left[\sum_{n=1}^{N} \phi(n) \phi^{T}(n)\right]^{-1}\left[\sum_{n=1}^{N}(\phi(n) y(n)]\right.
$$


To derive the RLS solution, we write equation 3.18 as a function of time and place weights on old data and take a single data point at $(t+1)$.

$$
\hat{\theta}_{L S}^{t}=\left[\sum_{i=1}^{t} \beta \phi(i) \phi^{T}(i)\right]^{-1}\left[\sum_{i=1}^{t} \beta \phi(i) y(i)\right]
$$

where $\beta=\lambda^{N-t}$ is the weight allows us track parameter changes by placing less weights on older data points and $\lambda$ is the forgetting factor. Typically $\lambda$ is set as $0.95 \leq \lambda \leq 1$, if $\lambda$ is set close to 0.95 then old data is forgotten very quickly. Let us define

$$
R(t)=\sum_{i=1}^{t} \beta \phi(i) \phi^{T}(i)
$$

and

$$
f(t)=\sum_{i=1}^{t} \beta \phi(i) y(i)
$$

Then at time $t$

$$
\hat{\theta}_{L S}(t)=R^{-1}(t) f(t)
$$

at time step $t+1$

$$
\begin{gathered}
R(t+1)=\lambda R(t)+\phi(t+1) \phi^{T}(t+1) \\
f(t+1)=\lambda f(t)+\phi(t+1) y(t+1) \\
\hat{\theta}(t+1)=R^{-1}(t+1) f(t+1) \\
=R^{-1}(t+1)[\lambda R(t) \hat{\theta}+\phi(t+1) y(t+1)]
\end{gathered}
$$

from eq. 3.23

$$
R(t)=\frac{1}{\lambda}\left[R(t+1)-\phi(t+1) \phi^{T}(t+1)\right]
$$


substituting eq. 3.27 in eq. 3.26

$$
\begin{aligned}
\hat{\theta}(t+1) & =R^{-1}(t+1)\left[R(t+1) \hat{\theta}(t)-\phi(t+1) \phi^{T}(t+1) \hat{\theta}(t)+\phi(t+1) y(t+1)\right] \\
& =\hat{\theta}(t)+R^{-1}(t+1) \phi(t+1)\left[y(t+1)-\phi^{T}(t+1) \hat{\theta}(t)\right]
\end{aligned}
$$

the term $\left[y(t+1)-\phi^{T}(t+1) \hat{\theta}(t)\right]$ in eq. 3.29 is the Prediction Error. The RLS algorithm is not implemented in terms of $R^{-1}$,rather a new matrix $P=R^{-1}$ is defined. Recall from Eq $3.6 R$ is the information matrix, so $P=R^{-1}$ is the covariance matrix. The covariance matrix $P$ is implemented using the matrix inversion lemma. See 14,18

Lemma 3.1.1 (Matrix inversion lemma) Given matrices $A, B, C$ and $D$ one can define a relationship as follows 14, 18;

$$
(A-B C D)^{-1}=A^{-1}-A^{-1} B\left(D A^{-1} B A C^{-1}\right)^{-1} D A^{-1}
$$

setting $A=\lambda R(t), B=\phi(t+1), C=1$ and $D=\phi^{-1}(t+1)$. The covariance matrix $P(t+1)$ can be calculated as:

$$
P(t+1)=R^{-1}(t+1)=\frac{1}{\lambda}\left(P(t)-\frac{P(t) \phi(t) \phi^{T}(t) P(t)}{\lambda+\phi^{T}(t) P(t) \phi(t)}\right)
$$

From eq. 3.29 we can express $R^{-1}(t+1) \phi(t+1)$ as

$$
R^{-1}(t+1) \phi(t+1)=\frac{P(t) \phi(t+1)}{\lambda+\phi^{T}(t+1) P(t) \phi(t+1)}
$$


The recursive least squares equation is

$$
\hat{\theta}(t+1)=\hat{\theta}(t)+L(t+1)\left[Y(t+1)-\phi^{T}(t+1) \hat{\theta}(t)\right]
$$

where

$$
L(t+1)=\frac{P(t) \phi(t+1)}{\lambda+\phi^{T}(t+1) P(t) \phi(t+1)}
$$

and $\lambda \in(0,1]$, is the user defined forgetting factor, which specifies how quickly the algorithm discounts past sample data.

\subsection{How to apply RLS to Delay Identification}



Figure 3.1: UAV position

At each time step $t$ we can derive an equation that defines a straight line passing through a point on the UAV's trajectory by the coordinates $[x(t), y(t)]$ and the current commanded heading $\psi_{\text {command }}(t)$. The heading is an indication of the UAV's direction, so at every point in time we can tell where the UAV is and what direction it is headed. If we take any two points A and B (see figure 3.1], and define the equations of the lines passing through $\mathrm{A}$ and $\mathrm{B}$, then we can solve the two lines equations simultaneously to find the point of intersection. This point of intersection is the target waypoint $\left(x_{w p}, y_{w p}\right)$ which the UAV is heading to. 




Figure 3.2: Example 3.1: illustrates the straight lines passing through points A and $\mathrm{B}$. The arrow head indicates the heading and the dash lines indicate the straight line passing through points A and B

Example 3.1 Given point $A$ as $\left(x_{a}=2000 k m, y_{a}=4000 k m\right)$, point $B$ as $\left(x_{b}=\right.$ $\left.6000 \mathrm{~km}, y_{b}=6000 \mathrm{~km}\right)$ and current heading at points $A$ and $B$ as $\psi_{a}=50.19^{\circ}$ and $\psi_{b}=75.964^{\circ}$ respectively, see figure 3.2 We can define the equation of a straight line passing through point $A$ as follows:

$$
\left(y_{w p}-y_{a}\right)=\tan \psi_{a}\left(x_{w p}-x_{a}\right)
$$


substituting $x_{a}, y_{a}$ and $\psi_{a}$ we get,

$$
\left(y_{w p}-4000\right)=\tan 50.19^{\circ}\left(x_{w p}-2000\right)
$$

Then bringing the waypoint values to the left hand side we get,

$$
\tan 50.19^{\circ}\left(-x_{w p}\right)+y_{w p}=\tan 50.19^{\circ}\left(-x_{a}\right)+y_{a}
$$

setting $m=\tan \psi$

$$
-m_{a} x_{w p}+y_{w p}=-m_{a} x_{a}+y_{a}
$$

the equation of a straight line passing through point $A\left(x_{a}=2000 \mathrm{~km}, y_{a}=4000 \mathrm{~km}\right)$ with a $\psi_{a}=50.19^{\circ}$ heading is

$$
-1.1998 x_{w p}+y_{w p}=1600.4
$$

Similarly we define the straight line passing through point $B$ as follows:

$$
\left(y_{w p}-y_{b}\right)=\tan \psi_{b}\left(x_{w p}-x_{b}\right)
$$

substituting $x_{b}, y_{b}$ and $\psi_{b}$ we get,

$$
\left(y_{w p}-6000\right)=\tan 75.964^{\circ}\left(x_{w p}-6000\right)
$$

Then bringing the waypoint values to the left hand side and setting $m=\tan \psi$ we 
get,

$$
-m_{b} x_{w p}+y_{w p}=-m_{b} x_{b}+y_{b}
$$

the equation of a straight line passing through point $B\left(x_{b}=6000 \mathrm{~km}, y_{b}=6000 \mathrm{~km}\right)$ with a $\psi_{b}=75.964^{\circ}$ heading is

$$
-4 x_{w p}+y_{w p}=-18000
$$

Solving eq 3.33 and 3.37 gives us

$$
\begin{gathered}
-1.1998 x_{w p}+y_{w p}=1600.4 \\
-4 x_{w p}+y_{w p}=-18000 \\
{\left[\begin{array}{ll}
-m_{1} & 1 \\
-m_{2} & 1
\end{array}\right]\left[\begin{array}{l}
x_{w p} \\
y_{w p}
\end{array}\right]=\left[\begin{array}{l}
\left(-m_{1} x_{1}+y_{1}\right) \\
\left(-m_{2} x_{2}+y_{2}\right)
\end{array}\right]} \\
=\left[\begin{array}{rr}
-1.1998 & 1 \\
-4 & 1
\end{array}\right]\left[\begin{array}{l}
x_{w p} \\
y_{w p}
\end{array}\right]^{\left[\begin{array}{l}
1600.4 \\
-18000
\end{array}\right]} \\
{\left[\begin{array}{l}
x_{w p} \\
y_{w p}
\end{array}\right]=\left[\begin{array}{rr}
-1.1998 & 1 \\
-4 & 1
\end{array}\right]^{-1}\left[\begin{array}{l}
1600.4 \\
-18000
\end{array}\right]}
\end{gathered}
$$




$$
\left[\begin{array}{l}
x_{w p} \\
y_{w p}
\end{array}\right]=\left[\begin{array}{l}
6999.999 \\
9999.999
\end{array}\right] \approx\left[\begin{array}{c}
7000 \\
10000
\end{array}\right]
$$

$x_{w p} \approx 7000 k m, y_{w p} \approx 10000 . x_{w p}$ and $y_{w p}$ are the coordinates of the point of intersection of the lines passing through point $\mathrm{A}$ and point $\mathrm{B}$. If $\mathrm{A}$ is the UAV location at time $t_{1}$ and $\mathrm{B}$ is the UAV location at time $t_{2},\left(x_{w p}, y_{w p}\right)$ is the coordinate of the target waypoint that the UAV is trying to reach. We can define the equation of a line passing through the UAV location at any time $t$ from eq 3.33 and 3.37 as

$$
-m(t) x_{w p}+y_{w p}=-m(t) x_{\text {uav }}(t)+y_{\text {uav }}(t)
$$

where, $\left(x_{w p}, y_{w p}\right)=$ xy-coordinate of the waypoint, $m(t)=\tan \psi_{\text {command }}(t)$ and $\left(x_{\text {uav }}(t), y_{\text {uav }}(t)\right)=\mathrm{UAV}$ xy-coordinate at time $\mathrm{t}$.

The operator heading command $\psi_{\text {command }}(t)$, is generated based on the operator's knowledge of the UAV coordinates at time $t$ and the target waypoint $\left(x_{w p}, y_{w p}\right)$. The operator generates a heading command using eq 2.20 as

$$
\psi_{\text {command }}(t)=\tan ^{-1}\left(\frac{y_{w p}-y_{\text {uav }}(t)}{x_{w p}-x_{\text {uav }}(t)}\right)
$$


from figure 3.2

$$
\begin{aligned}
& \psi_{a}=\tan ^{-1}\left(\frac{10000-4000}{7000-2000}\right) \\
& \psi_{a}=50.1944^{\circ} \\
& \psi_{b}=\tan ^{-1}\left(\frac{10000-6000}{7000-6000}\right) \\
& \psi_{b}=75.9638^{\circ}
\end{aligned}
$$

The heading command at time $t$ is matched to the UAV coordinates $\left(x_{\text {uav }}(t)\right.$, $\left.y_{\text {uav }}(t)\right)$. If there is a mismatch between the heading command and the UAV coordinates (i.e matching a heading command with a different location), we will get a different waypoint.

Example 3.2 We consider the case where the heading commands $\psi_{a}$ and $\psi_{b}$ are executed in locations with coordinates that are a mismatch to the heading commands. As illustrated in figure 3.3 , we see that the lines passing through point A and point $\mathrm{B}$ will intersect at a location $M$ which is different from the target waypoint $\left(x_{w p}, y_{w p}\right)$. The UAV will be heading in a different direction. 


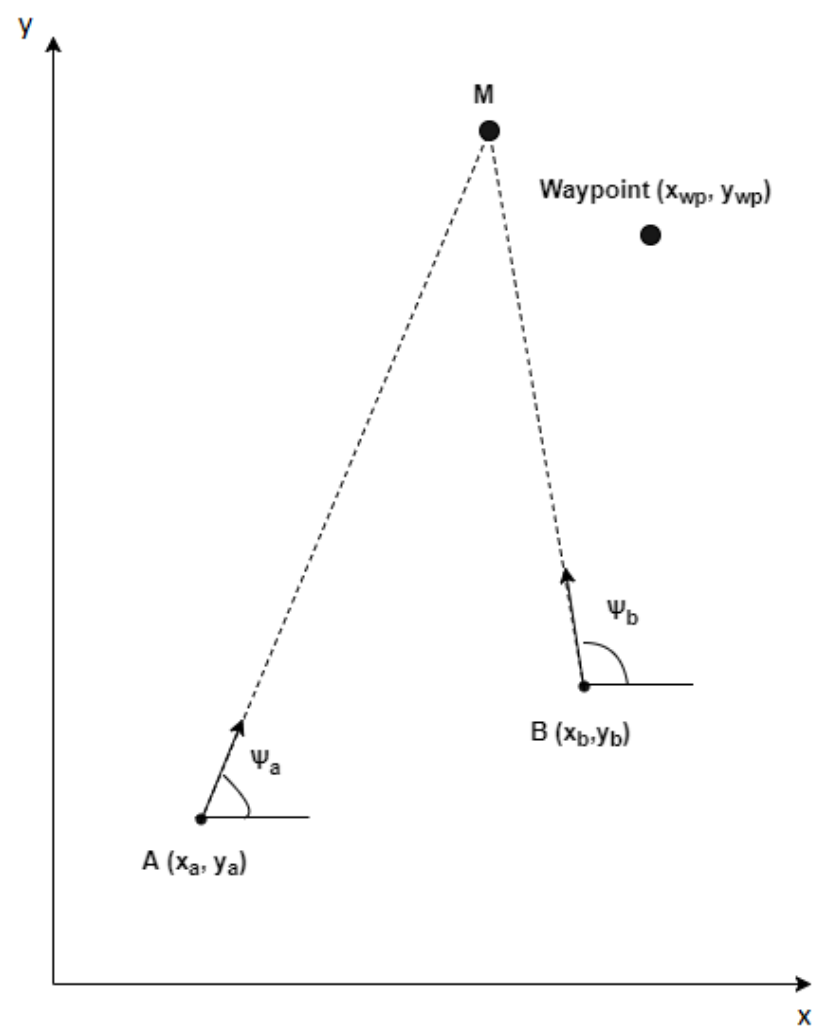

Figure 3.3: Example 3.2: straight lines passing through points A and B illustrating the effects of mismatch between heading angle $\psi$ and UAV coordinates $\left(x_{\text {uav }}\right.$, $\left.y_{\text {uav }}\right)$. The arrow head indicates the heading and the dash lines indicate the straight line passing through the UAV location at A and B

With time delays in the heading command, when the operator sends a heading command at time $t$ based on the UAV location $\left(x_{\text {uav }}(t), y_{\text {uav }}(t)\right)$ the UAV receives the command at time $(t+$ delay $)$ when the UAV is in a different location $\left(x_{\text {uav }}(t+\right.$ delay $)$, $y_{\text {uav }}(t+$ delay $\left.)\right)$. This means that the command the UAV receives is a mismatch to the UAV's location when it receives the command and as such the UAV will head in a different direction. This is illustrated in figure 3.4 . 


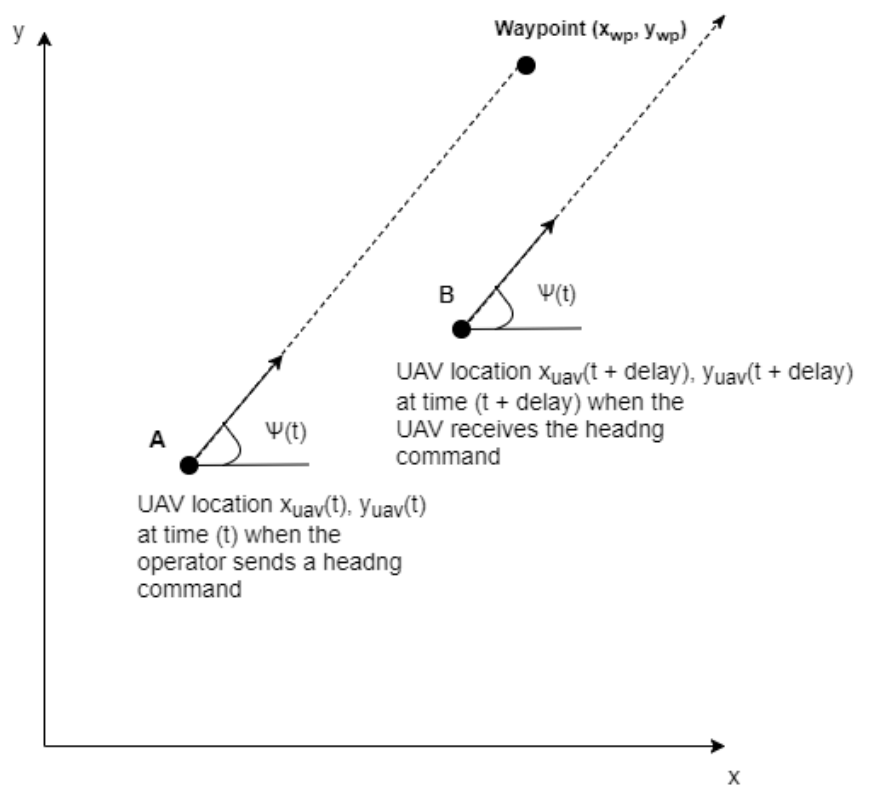

Figure 3.4: $A$ is the UAV location when the command was sent, B is the UAV location when the same command is received. The arrow head indicates the heading and the dash lines indicate the straight line passing through UAV location at $\mathrm{A}$ and $\mathrm{B}$

To get the coordinates of the location the UAV was when the heading command was sent, i.e the UAV coordinates that match with the received commands, one will have to look at the coordinates at $d$ time steps back (where $d=$ delay). If we assume that the heading command is delayed by 5 time steps, then one looks at the coordinates 5 time steps back every time a command is received in order to have an accurate estimation of the target waypoint.

If we take $n$ points on the UAV's trajectory as illustrated in figure 3.5 




Figure 3.5: Illustrating points at different time steps $t$ on the UAV trajectory. The arrow head indicates the heading and the black dash lines indicate the straight line passing through points at different time steps $t$ and the red dash curves is the UAV trajectory.

using eq. 3.39 we can define the straight lines passing through these points at time step $t_{1}$ to $t_{n}$ as:

$$
\begin{aligned}
-m\left(t_{1}\right) x_{\text {uav }}\left(t_{1}\right)+y_{\text {uav }}\left(t_{1}\right) & =-m\left(t_{1}\right) x_{w p}+y_{w p} \\
-m\left(t_{2}\right) x_{\text {uav }}\left(t_{2}\right)+y_{\text {uav }}\left(t_{2}\right) & =-m\left(t_{2}\right) x_{w p}+y_{w p} \\
\vdots & =\vdots \\
-m\left(t_{n}\right) x_{\text {uav }}\left(t_{n}\right)+y_{\text {uav }}\left(t_{n}\right) & =-m\left(t_{n}\right) x_{w p}+y_{w p}
\end{aligned}
$$

where $m\left(t_{n}\right)=\tan \psi\left(t_{n}\right)$ which is the heading command at time step $t_{n}$, $\left(x_{\text {uav }}\left(t_{n}\right), y_{\text {uav }}(t),\right)$ is the UAV coordinate at time step $t_{n}$. The point $\left(x_{w p}, y_{w p}\right)$ is coordinate of the target waypoint. To solve for $x_{w p}$ and $y_{w p}$, we use the LS algorithm. 
Then we can write

$$
Y=\Phi^{T} \Theta
$$

where the matrices $\Phi, Y$ and $\Theta$ are

$$
\begin{aligned}
& \Phi^{T}=\left[\begin{array}{cc}
-m\left(t_{1}\right) & 1 \\
-m\left(t_{2}\right) & 1 \\
-m\left(t_{3}\right) & 1 \\
\vdots & \vdots \\
-m\left(t_{n}\right) & 1
\end{array}\right] \\
& Y=\left[\begin{array}{c}
\left(-m\left(t_{1}\right) x_{\text {uav }}\left(t_{1}\right)+y_{\text {uav }}\left(t_{1}\right)\right) \\
\left(-m\left(t_{2}\right) x_{\text {uav }}\left(t_{2}\right)+y_{\text {uav }}\left(t_{2}\right)\right) \\
\vdots \\
\left(-m\left(t_{n}\right) x_{u a v}\left(t_{n}\right)+y_{\text {uav }}\left(t_{n}\right)\right)
\end{array}\right]
\end{aligned}
$$

from eq 3.16 the LS estimate $\hat{\Theta}_{L S}$ is

$$
\hat{\Theta}_{L S}=\left[\begin{array}{l}
x_{w p} \\
y_{w p}
\end{array}\right]=\left(\Phi \Phi^{T}\right)^{-1} \Phi Y .
$$

Example 3.3 We consider a UAV being controlled to reach a waypoint with the coordinate $(2000,5000)$. An operator remotely generates commands $\psi\left(t_{n}\right)$ based 
on the UAV coordinates at each time step using eq 2.20 . We are given the xycoordinates to five points on the UAV trajectory as:

$$
\begin{gathered}
x_{\text {uav }}=\left[\begin{array}{lllll}
130.9396 & 137.4866 & 144.0335 & 150.5805 & 157.1275
\end{array}\right] \\
y_{\text {uav }}=\left[\begin{array}{lllll}
-19.8515 & -20.8440 & -21.8366 & -22.8291 & -23.8215
\end{array}\right]
\end{gathered}
$$

Assuming that there are no delays in the input commands (i.e the coordinates match the received commands), let us find the target waypoint from the UAV coordinates and the received heading commands. Taking the equations of the straight lines passing through each of the the xy-coordinates and setting $m\left(t_{n}\right)=\tan \psi\left(t_{n}\right)$, we define $\Phi$ and $Y$ from eq 3.42 and 3.43 as:

$$
\begin{aligned}
& \Phi^{T}=\left[\begin{array}{ll}
-m\left(t_{1}\right) & 1 \\
-m\left(t_{2}\right) & 1 \\
-m\left(t_{3}\right) & 1 \\
-m\left(t_{4}\right) & 1 \\
-m\left(t_{5}\right) & 1
\end{array}\right]=\left[\begin{array}{ll}
-2.6369 & 1 \\
-2.6466 & 1 \\
-2.6563 & 1 \\
-2.6660 & 1 \\
-2.6759 & 1
\end{array}\right] \\
& Y=\left[\begin{array}{l}
\left(-m\left(t_{1}\right) x_{\text {uav }}\left(t_{1}\right)+y_{\text {uav }}\left(t_{1}\right)\right) \\
\left(-m\left(t_{2}\right) x_{\text {uav }}\left(t_{2}\right)+y_{\text {uav }}\left(t_{2}\right)\right) \\
\left(-m\left(t_{3}\right) x_{\text {uav }}\left(t_{3}\right)+y_{\text {uav }}\left(t_{3}\right)\right) \\
\left(-m\left(t_{4}\right) x_{\text {uav }}\left(t_{4}\right)+y_{\text {uav }}\left(t_{4}\right)\right) \\
\left(-m\left(t_{5}\right) x_{\text {uav }}\left(t_{5}\right)+y_{\text {uav }}\left(t_{5}\right)\right)
\end{array}\right]=\left[\begin{array}{l}
-273.8469 \\
-293.1124 \\
-312.5114 \\
-332.0455 \\
-351.7158
\end{array}\right]
\end{aligned}
$$




$$
\left(\Phi \Phi^{T}\right)^{-1}=\left[\begin{array}{cc}
1055.5 & 2803.7 \\
2803.7 & 7447.7
\end{array}\right]
$$

$$
\Phi Y=\left[\begin{array}{c}
4154.3 \\
-1563.2
\end{array}\right]
$$

The least squares estimate $\hat{\Theta}_{L S}$ gives us the coordinates to the waypoint and it is calculated as:

$$
\hat{\Theta}_{L S}=\left[\begin{array}{c}
x_{w p} \\
y_{w p}
\end{array}\right]=\left(\Phi \Phi^{T}\right)^{-1} \Phi Y=\left[\begin{array}{c}
2000 \\
5000
\end{array}\right]
$$

To see how good the estimate $\hat{\Theta}$ is, we calculate the prediction errors using eq 3.17

$$
E=Y-\Phi^{T} \Theta=\left[\begin{array}{l}
-273.8469 \\
-293.1124 \\
-312.5114 \\
-332.0455 \\
-351.7158
\end{array}\right]-\left[\begin{array}{ll}
-2.6369 & 1 \\
-2.6466 & 1 \\
-2.6563 & 1 \\
-2.6660 & 1 \\
-2.6759 & 1
\end{array}\right]\left[\begin{array}{c}
2000 \\
5000
\end{array}\right]
$$


$x_{w p}=2000$ and $y_{w p}=5000$

$$
=\left[\begin{array}{l}
-0.1300 \\
-0.1459 \\
-0.1639 \\
-0.1792 \\
-0.1969
\end{array}\right] \times 10^{-8}
$$

The prediction errors are almost zero indicating that we have a good estimate of the target waypoint.

\subsubsection{Bank of Least Squares Filters}

We are now able to estimate a target waypoint given a set of coordinates and respective heading commands using the least squares algorithm. Let one assume that an operator at time $t=0$, believes the $\mathrm{UAV}$ is at a location $\left(x_{\text {uav }}(0), y_{\text {uav }}(0)\right)$ and sends a command $\psi(t=0)$. However the UAV receives the the command at $t=5$ at a location $\left(x_{\text {uav }}(5), y_{\text {uav }}(5)\right)$. This means that there is a 5 seconds delay between when the operator sends the command and when the UAV receives the command. This is illustrated in figure 3.4 The commands the UAV receives at $t=5$ is a mismatch to the UAV coordinates at $\left(x_{\text {uav }}(5), y_{\text {uav }}(5)\right)$ and as such the UAV will head in a different direction from the operator's desired direction. Suppose one is able to keep track of the past coordinates such that when a command is received, the command is matched with the past coordinates to see which coordinates will predict the most suitable target waypoint in a least squares estimation (i.e minimize the prediction errors). From the illustration above, when the UAV receives the command at $\left(x_{\text {uav }}(5), y_{\text {uav }}(5)\right)$ it looks at its coordinates 
5 seconds back (i.e at $t=t-5$ ) to correctly match the received commands to the appropriate coordinates. The UAV has no a priori knowledge of the delay or its coordinates at the time the command was issued nor does it have a priori knowledge of the target waypoint. The UAV learns the target waypoint and the

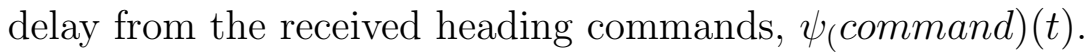

Suppose we design a bank of LS filters on the UAV controller. We match each filter to a different time delay, $d_{i}$. In this case, $d_{i}$ specifies how many steps into the past UAV coordinates that the $i$ th filter tracks. The filter structure as defined by eq. 3.39 is modified into

$$
-m(t) x_{w p}+y_{w p}=-m(t) x_{u a v}\left(t-d_{i}\right)+y_{u a v}\left(t-d_{i}\right)
$$

For $n$ points,

$$
\begin{gathered}
-m\left(t_{1}\right) x_{w p}+y_{w p}=-m\left(t_{1}\right) x_{\text {uav }}\left(t_{1}-d_{i}\right)+y_{\text {uav }}\left(t_{1}-d_{i}\right) \\
-m\left(t_{2}\right) x_{w p}+y_{w p}=-m\left(t_{2}\right) x_{\text {uav }}\left(t_{2}-d_{i}\right)+y_{\text {uav }}\left(t_{2}-d_{i}\right) \\
\vdots \\
-m\left(t_{n}\right) x_{w p}+y_{w p}=-m\left(t_{n}\right) x_{\text {uav }}\left(t_{n}-d_{i}\right)+y_{\text {uav }}\left(t_{n}-d_{i}\right)
\end{gathered}
$$


Then we have,

$$
\begin{aligned}
& \Phi_{i}^{T}=\left[\begin{array}{cc}
-m\left(t_{1}\right) & 1 \\
-m\left(t_{2}\right) & 1 \\
-m\left(t_{3}\right) & 1 \\
\vdots & \\
-m\left(t_{n}\right) & 1
\end{array}\right] \\
& Y_{i}=\left[\begin{array}{c}
\left(-m\left(t_{1}\right) x_{\text {uav }}\left(t_{1}-d_{i}\right)+y_{\text {uav }}\left(t_{1}-d_{i}\right)\right) \\
\left(-m\left(t_{2}\right) x_{\text {uav }}\left(t_{2}-d_{i}\right)+y_{\text {uav }}\left(t_{2}-d_{i}\right)\right) \\
\vdots \\
\left(-m\left(t_{n}\right) x_{\text {uav }}\left(t_{n}-d_{i}\right)+y_{\text {uav }}\left(t_{n}-d_{i}\right)\right)
\end{array}\right] \\
& \hat{\Theta}_{i}=\left[\begin{array}{l}
x_{w p} \\
y_{w p}
\end{array}\right]
\end{aligned}
$$

The the least squares solution for each of the $i$ th filter is

$$
\hat{\Theta}_{i L S}=\left[\Phi_{i}(t) \Phi_{i}(t)^{T}\right]^{-1}\left[\Phi_{i}(t) Y_{i}(t)\right]
$$

The prediction error is

$$
\epsilon=\left[Y_{i}-\Phi_{i}^{T} \hat{\Theta}_{i L S}\right]
$$

Where $n$ is the sample size, $d_{i}$ is the time step of the $i$ th filter. 
As an example, lets add filters to the aircraft system in chapter 2. We design four filters setting $d_{i}=5,10,15,20$ time steps. The simulation is started after 20 points, i.e after we have accumulated $n=20$ samples. There after the number of samples for the LS solution is increased by 1 with each point until the UAV reaches the waypoint. The UAV is assumed to reach the waypoint when it gets to within a user defined threshold distance from the waypoint. In figure 3.6, the last point before the UAV reaches waypoint $A(2000,5000)$ is 1400 steps, so the least squares is based on 1400 points, i.e 1400 samples. The waypoints are placed at

$$
\begin{aligned}
x_{\text {waypoint }} & =\left[\begin{array}{lllllll}
0 & 2000 m & 7000 m & 15000 m & 18000 m & 12000 m & 2000 m
\end{array}\right] \\
y_{\text {waypoint }} & =\left[\begin{array}{lllllll}
0 & 5000 m & 8000 m & 8000 m & 4000 m & 200 k m & 10000 m
\end{array}\right]
\end{aligned}
$$

and the simulation is continued until the UAV reaches within the user defined threshold of the first way point A $(2000,5000)$. The threshold radius is selected as $500 \mathrm{~m}$.

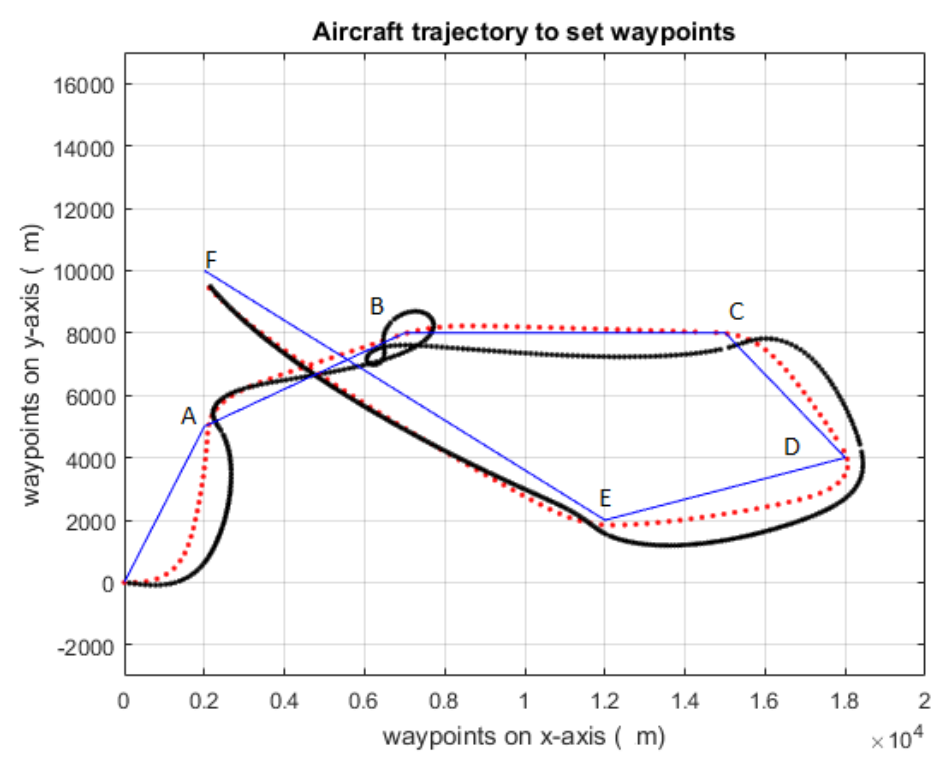

Figure 3.6: UAV trajectory with $2 \mathrm{~s}$ delayed input commands as it moves from waypoint $A$ to $F$. The red dotted line indicates the aircraft trajectory with no delay, the blue line is the straight line between waypoints and the black line indicates the UAV trajectory subjected to time delay. 
The figure above illustrates the trajectory of the UAV receiving heading commands subjected to 20 time step delay which is 2 seconds. Four LS filters each matched to time delays of $0.5,1.0,1.5$ and 2 seconds which is $d_{i}=5,10,15$ and 20 time steps respectively are set up. The mathematical model of the filters are defined using eq. 3.51 and the LS parameters using eq. $3.52,3.53$ and 3.54 Each LS filter $i$ stores $n$ data points of specified past UAV coordinates, for example the 5 step LS filter (i.e $\left.d_{i}=5\right)$ stores the UAV coordinates of 5 time step back (i.e $x_{\text {uav }}(t-5)$, yuav $(t-5)$ ). The filter that most suitably estimates the target waypoint determines what the time delay is. If the estimates of the 5 step LS filter is most suitable, then we have a 5 step time delay which is 0.5 seconds. The prediction errors tell us which filter has the most suitable estimate of the waypoint. Typically, the prediction errors are small, close to zero and appear as white noise.

\subsubsection{Simulation results:}

The waypoint we want to estimate is at A $(2000,5000)$ see figure 3.6 Figures 3.7 - 3.14 illustrates the waypoint estimates and the prediction errors of the four LS filters for 600 time steps. The true delay is 2 seconds or 20 time steps. 




Figure 3.7: Least squares waypoint estimate of the 5 step ( 0.5 seconds) LS Filter. $x_{w p}$ (blue line) converges to approximately 2030 and $y_{w p}$ (red line) converges to approximately 4800 . The true delay is 2 seconds and the true waypoint coordinate is $(2000,5000)$.



Figure 3.8: The prediction errors of 5 step (0.5 seconds) LS Filter show that the errors are not close to zero. The LS estimate of the 5 step ( 0.5 second) LS filter is not a correct estimation of the target waypoint. 


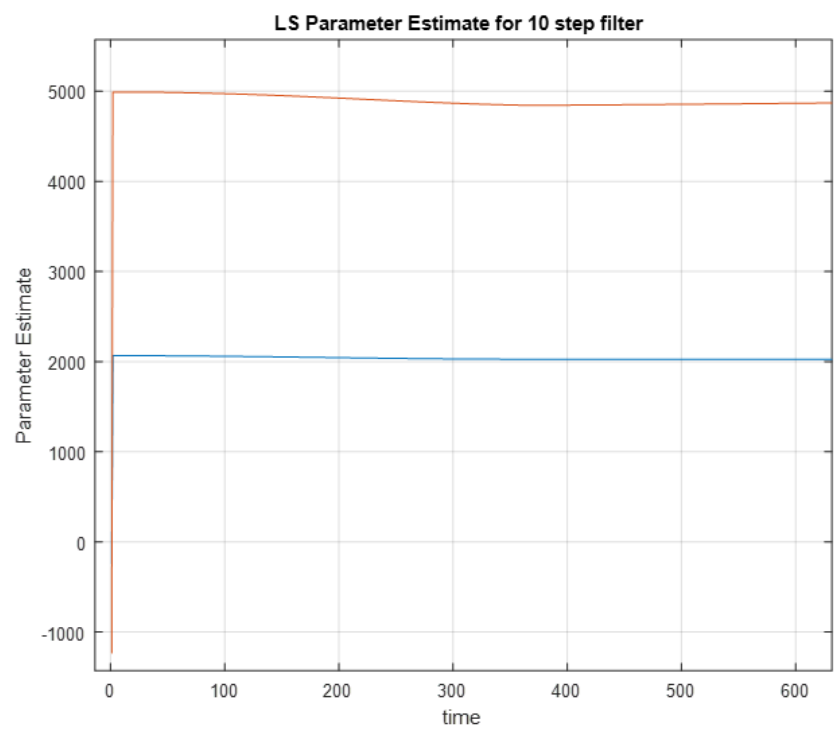

Figure 3.9: Least squares waypoint estimate of the 10 step LS Filter. $x_{w p}$ (blue line) converges to approximately 2020 and $y_{w p}$ (red line) converges to approximately 4860 . The true delay is 2 seconds and the true waypoint coordinate is $(2000,5000)$.



Figure 3.10: The prediction errors of 10 step (1 second) LS Filter show that the errors are not close to zero. The LS estimate of the 10 step (1 second) LS filter is not a correct estimation of the waypoint. 




Figure 3.11: Least squares waypoint estimate of the 15 step LS Filter. $x_{w p}$ (blue line) converges to approximately 2013 and $y_{w p}$ (red line) converges to approximately 4900. The true delay is 2 seconds and the true waypoint coordinate is $(2000,5000)$.

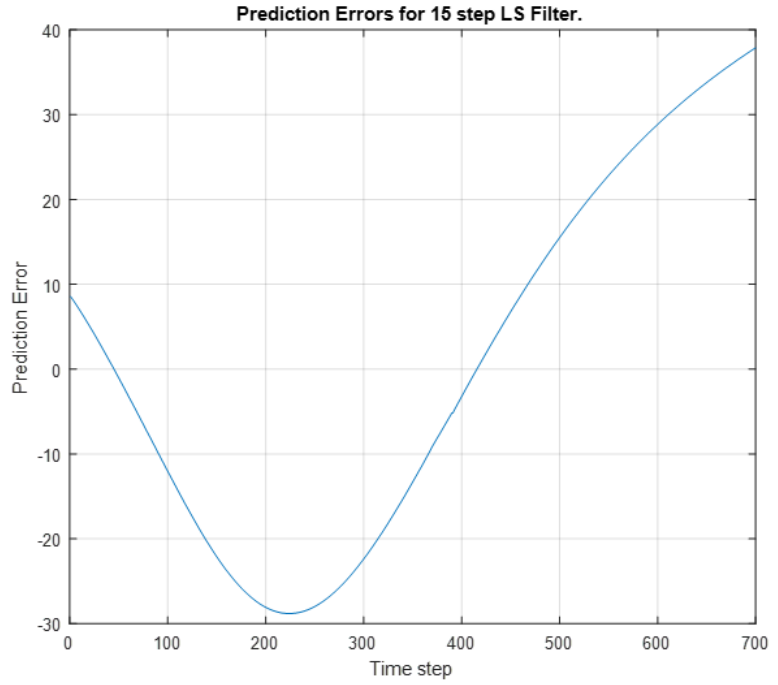

Figure 3.12: The prediction errors of 15 step (1.5 seconds) LS Filter show that the errors are not close to zero. The LS estimate of the 15 step (1.5 seconds) LS filter is not a correct estimation of the waypoint. 


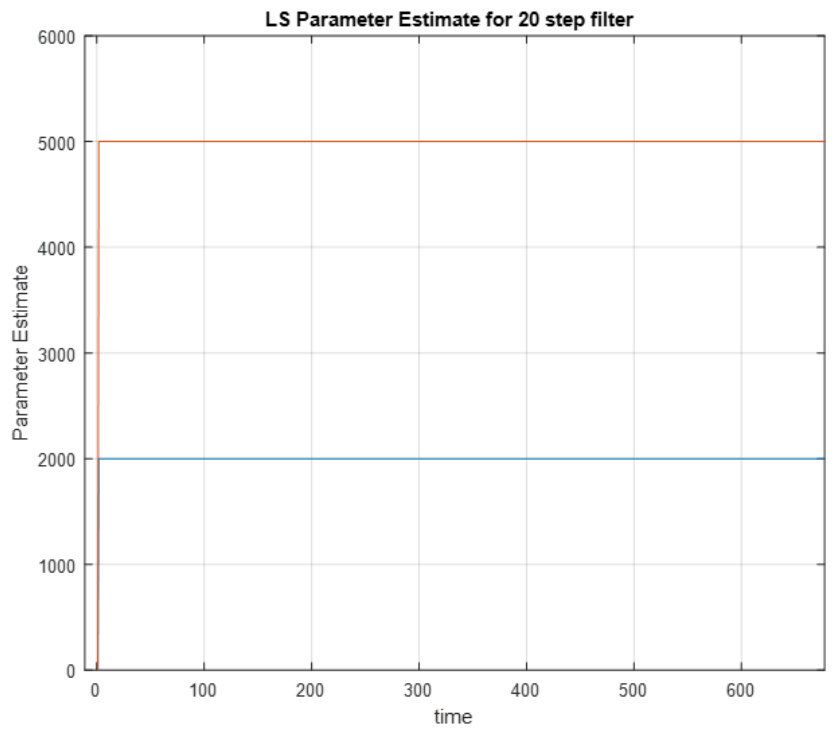

Figure 3.13: Least squares waypoint estimate of the 20 step LS Filter. $x_{w p}$ (blue line) converges to approximately 2000 and $y_{w p}$ (red line) converges to approximately 5000. This is the true delay ( 2 seconds) and the true waypoint coordinate.

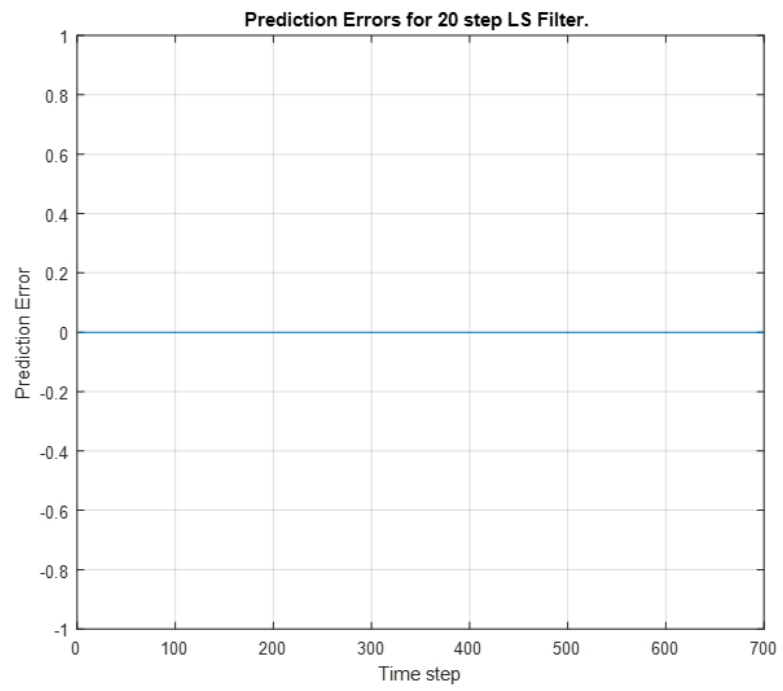

Figure 3.14: The prediction errors of 20 step LS Filter show that the errors are close to zero. The LS estimate of the 20 step (2 seconds) LS filter is accepted as a correct estimation of the waypoint and the time delay is identified as 2 seconds.

Comparing $x_{w p}$ and $y_{w p}$ of the four LS filters we get, 


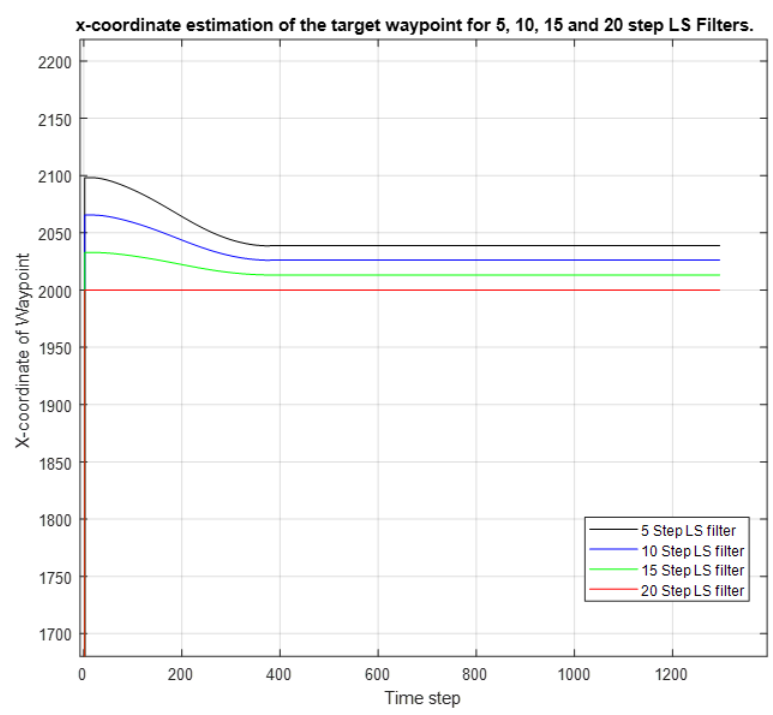

Figure 3.15: $x_{w p}$ LS estimate of the target waypoint. The black line shows the estimate of the 5 step ( 0.5 second)LS filter, the blue line shows the estimate of the 10 step (1 second) LS filter, the green line shows the estimate of the 15 step (1.5 seconds) LS filter and the red line shows the estimate of the 20 step (2 seconds) LS filter.

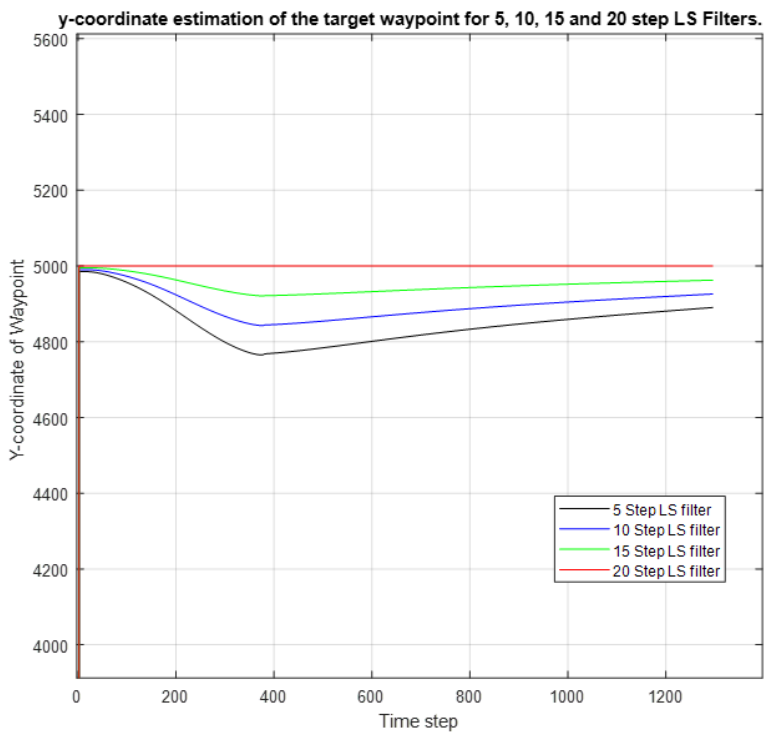

Figure 3.16: $y_{w p}$ LS estimate of the target waypoint. The black line shows the estimate of the 5 step ( 0.5 second) LS filter, the blue line shows the estimate of the 10 step (1 second) LS filter, the green line shows the estimate of the 15 step (1.5 seconds) LS filter and the red line shows the estimate of the 20 step (2 seconds) LS filter. 
Comparing the prediction errors of the four filters in figure 3.17, we note the differences in the errors and we can see that the prediction errors of the 20 step LS filter converges to zero.



Figure 3.17: Prediction errors of the four LS filters. The black line shows the errors of the 5 step LS filter, the blue line shows the errors of the 10 step LS filter, the green line shows the errors of the 15 step LS filter and the red line shows the errors of the 20 step LS filter.

We observe that the estimates $\hat{\theta}_{i}$ of the 5, 10 and 15 step LS filters do not converge to the target waypoint coordinates (figures 3.7, 3.9 and 3.11). This is because the past coordinates tracked by the 5, 10 and 15 step filters do not match accurately with the received heading command. The prediction errors in figures $3.8,3.10$ and 3.12 are not close to zero which tells us that the estimates of the 5, 10 and 15 step LS filters are not a good fit for the target waypoint estimate.

The coordinates of the 20 step LS filter accurately matches with the input 


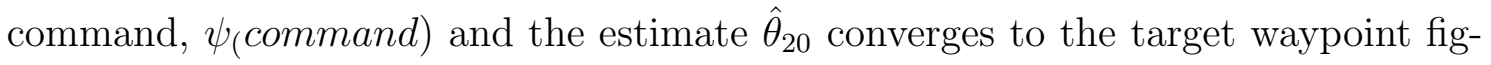
ure 3.13 . When we look at the prediction errors in figure 3.14 , the errors are close to zero. This confirms the goodness of fit of the 20 step LS filter waypoint estimate.

We can say that with a close to zero prediction error of the target waypoint, $\psi_{\text {command }}$ correctly matches with the coordinates at 20 steps back from the current UAV location and the estimate $\hat{\theta}_{20}$ is a good estimate for the target waypoint. Therefore the delay in receiving the input commands is identified as 20 time steps which is equivalent to a 2 seconds delay.

\subsubsection{Bank of Recursive Least Squares Filters}

In the least squares filters above, keeping track of past coordinates requires having to store the data. This presents a drawback with the LS filter design because of the demand for memory. In Recursive Least Squares one can select an appropriate for-

getting factor $\lambda \in(0,1]$, that specifies the number of data points to be considered for estimation and discards unwanted data. The estimation is based on approximately $1 /(1-\lambda)$ data points.

The LS Filter design above is implemented using the RLS algorithm discussed in section 3.1.2 and selecting $\lambda=0.95$, as such the estimate is based on approximately 20 data points

First, we assume a solution to the least squares estimate of the target waypoint by initializing $\hat{\theta}$, to zero.

$$
\hat{\theta}_{i L S}=[0,0]^{T}
$$

where $\hat{\theta}_{i L S}$ is the least squares estimate of the $i$ th filter. Then the covariance matrix 
$P_{i}$ is initialized to a large diagonal matrix.

$$
P_{i}(t)=\left[\begin{array}{cc}
1000 & 0 \\
0 & 1000
\end{array}\right]
$$

Let us implement the least squares filters discussed in the previous section using the recursive least squares algorithm. Four RLS filters each matched to time delay of $0.5,1.0,1.5$ and 2 seconds which is $d_{i}=5,10,15$ and 20 time steps respectively are set up. Each filter $i$ tracks the UAV past coordinates in $d_{i}$ time steps. For example the 5 step RLS filter (i.e $d_{i}=5$ ) tracks the UAV coordinates of 5 time steps back (i.e $x_{\text {uav }}(t-5), y_{\text {uav }}(t-5)$ ). The received commands at time $t$ are matched with the past coordinates at $t-d_{i}$ and the combination is used to define the line equation passing through the UAV coordinates (see figure 3.5) at each time(t) using eq 3.2.1. The RLS algorithm is defined for each filter $i$ as follows:

$$
\begin{aligned}
\Phi_{i} & =\left[\begin{array}{ll}
-m(t) & 1
\end{array}\right] \\
Y_{i} & =\left[\left(-m(t) x_{\text {uav }}\left(t-d_{i}\right)+y_{\text {uav }}\left(t-d_{i}\right)\right)\right] \\
L_{i}(t) & =\frac{P_{i}(t) \Phi_{i}(t)}{\lambda+\Phi_{i}^{T}(t) P_{i}(t) \Phi_{i}(t)} \\
\epsilon(t) & =\left[Y-\Phi^{T}(t) \hat{\Theta}(t)\right]
\end{aligned}
$$

where $m=\tan \psi_{\text {command }}(t)$. Compute update to the parameter estimate.

$$
\hat{\Theta}(t+1)=\hat{\Theta}(t)+L_{i}(t) \epsilon(t)
$$


Compute update to the covariance matrix.

$$
P_{i}(t+1)=\frac{1}{\lambda}\left(P_{i}(t)-L_{i}(t) \Phi_{i}(t) P_{i}(t)\right)
$$

The prediction errors tell us which filter has the most accurate estimate of the waypoint. Typically, the prediction errors are small, close to zero and appear as white noise.

Figures 3.18 - 3.25 illustrate the target waypoint estimates and prediction errors of the four recursive least squares filters. The waypoint we want to estimate is at A $(2000,5000)$ see figure 3.6 .

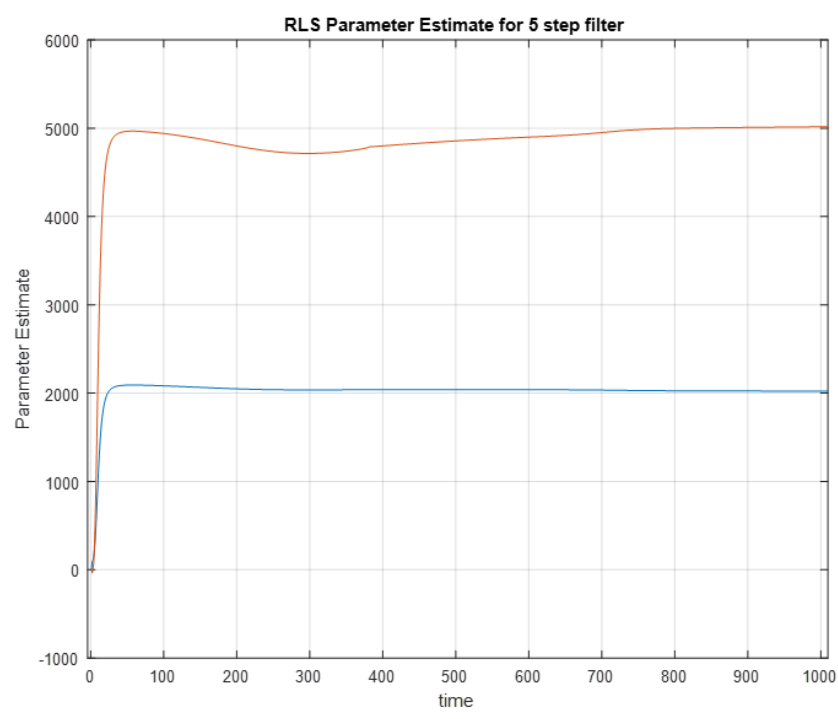

Figure 3.18: Target waypoint estimate of the 5 step ( 0.5 second) RLS Filter. $x_{w p}$ (blue) converges to approximately $2037.6 \mathrm{~km}$ and $y_{w p}$ (red) converges to approximately $4921.8 \mathrm{~km}$. 


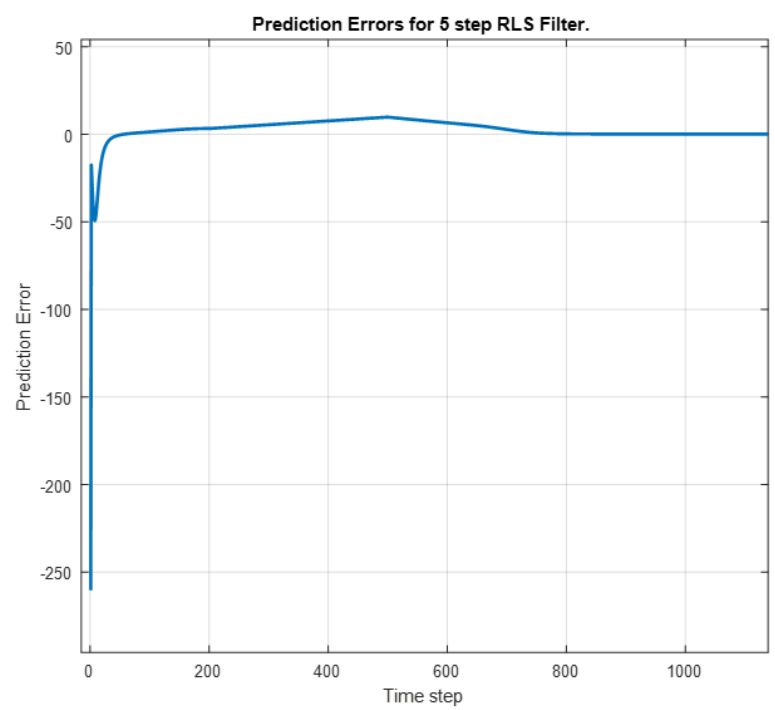

Figure 3.19: Prediction Errors of the 5 step RLS Filter.

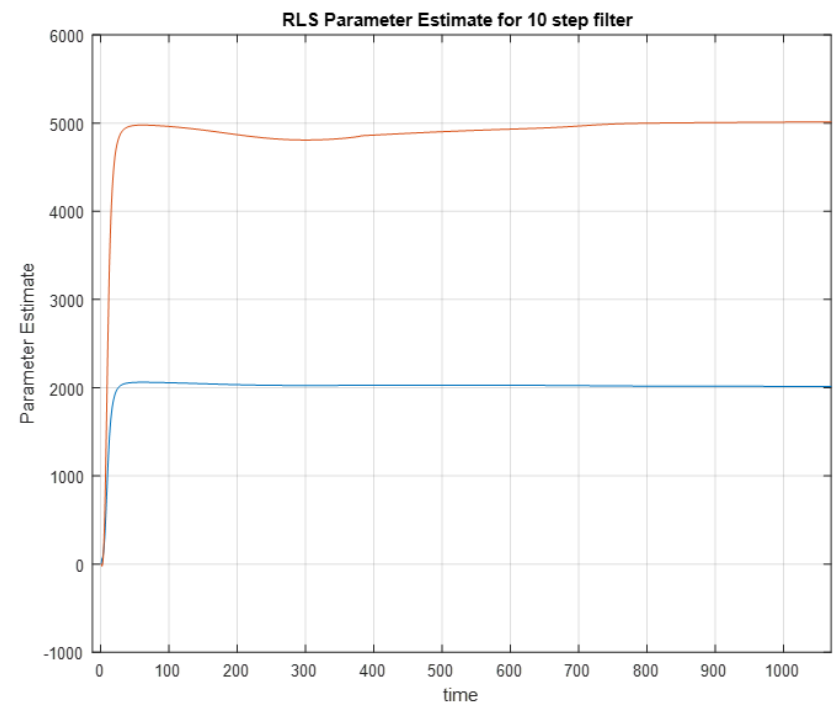

Figure 3.20: Target waypoint estimate of the 10 (1 second) step RLS Filter. $x_{w p}$ (blue) converges to approximately $2025.3 \mathrm{~km}$ and $y_{w p}$ (red) converges to approximately $4946.6 \mathrm{~km}$. 


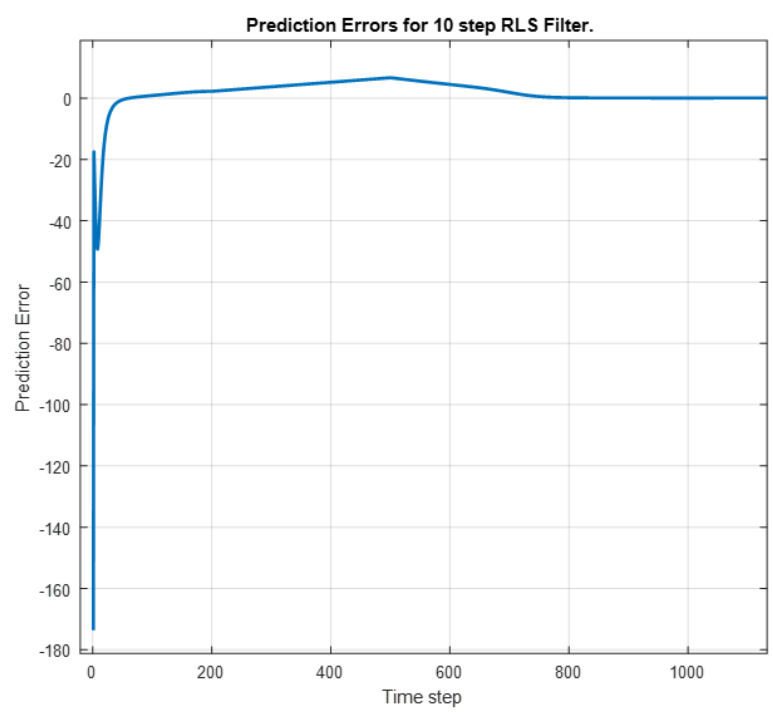

Figure 3.21: Prediction Errors of the 10 step RLS Filter.

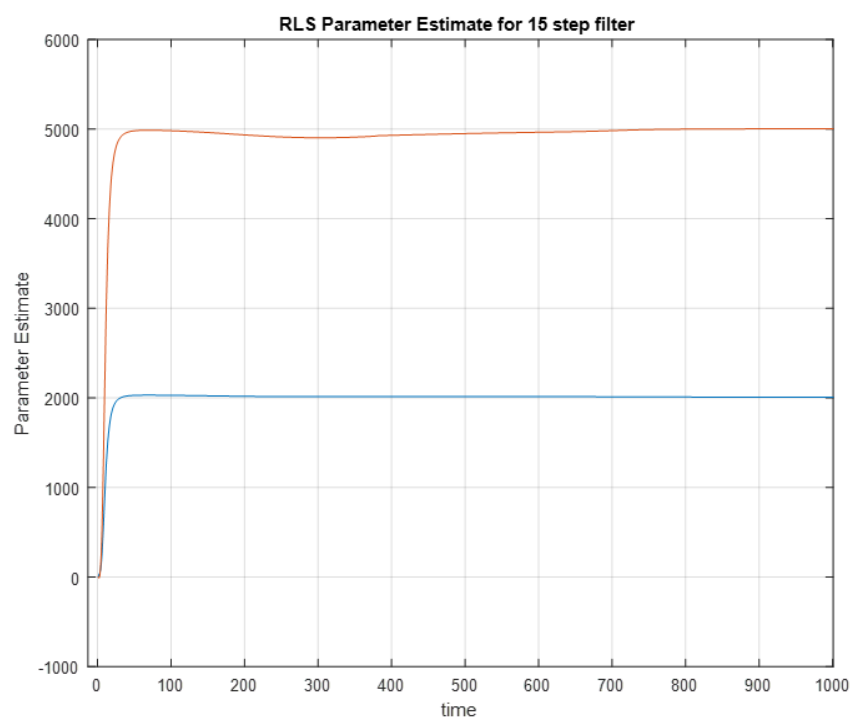

Figure 3.22: Target waypoint estimate of the 15 step (1.5 seconds) RLS Filter. $x_{w p}$ (blue) converges to approximately $2013.2 \mathrm{~km}$ and $y_{w p}$ (red) converges to approximately $4958.5 \mathrm{~km}$. 


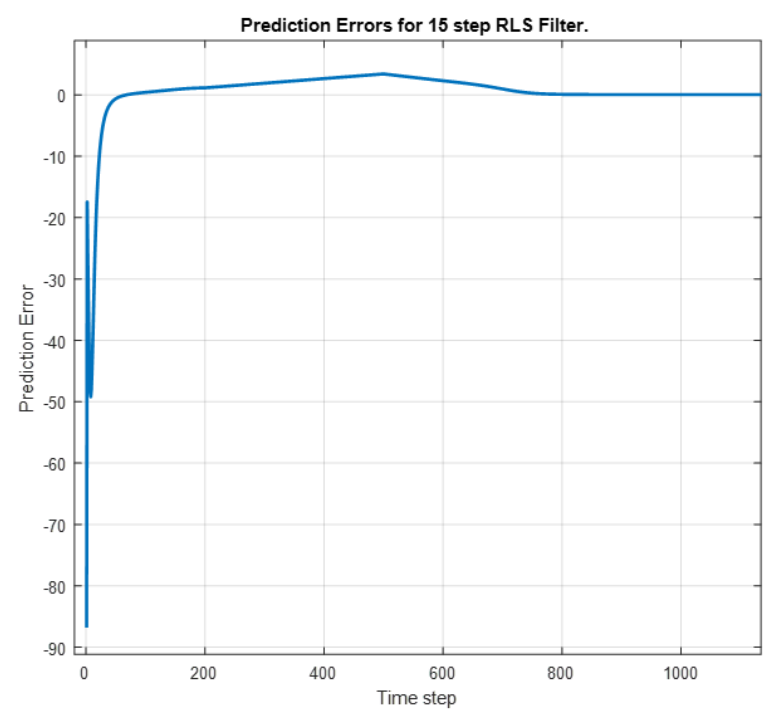

Figure 3.23: Prediction Errors of the 15 step RLS Filter.

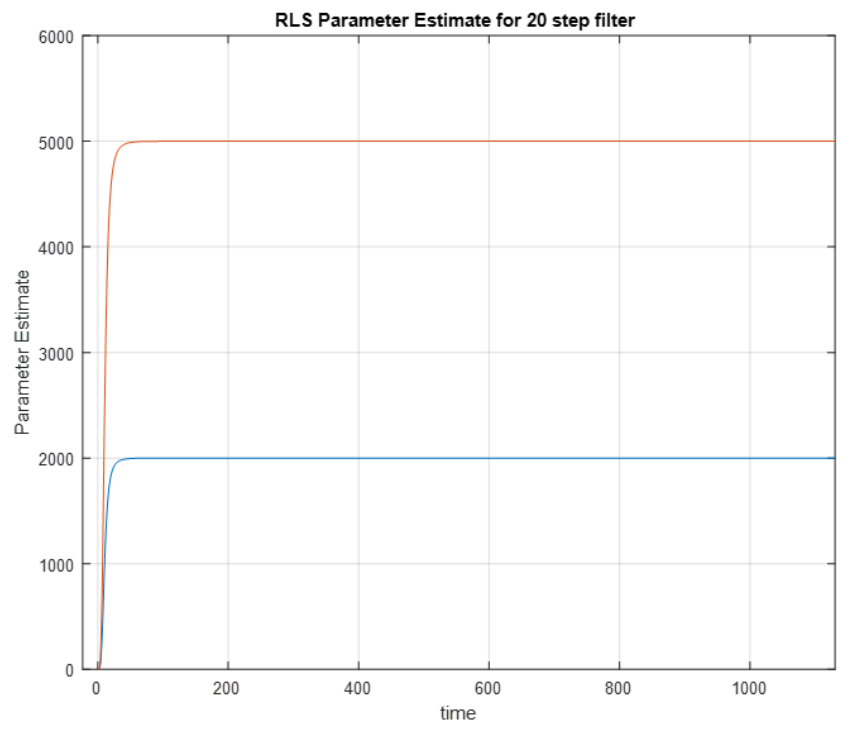

Figure 3.24: Target waypoint estimate of the 20 step (2 seconds) RLS Filter. $x_{w p}$ (blue) converges to approximately $2000 \mathrm{~km}$ and $y_{w p}$ (red) converges to approximately $5000 \mathrm{~km}$. 


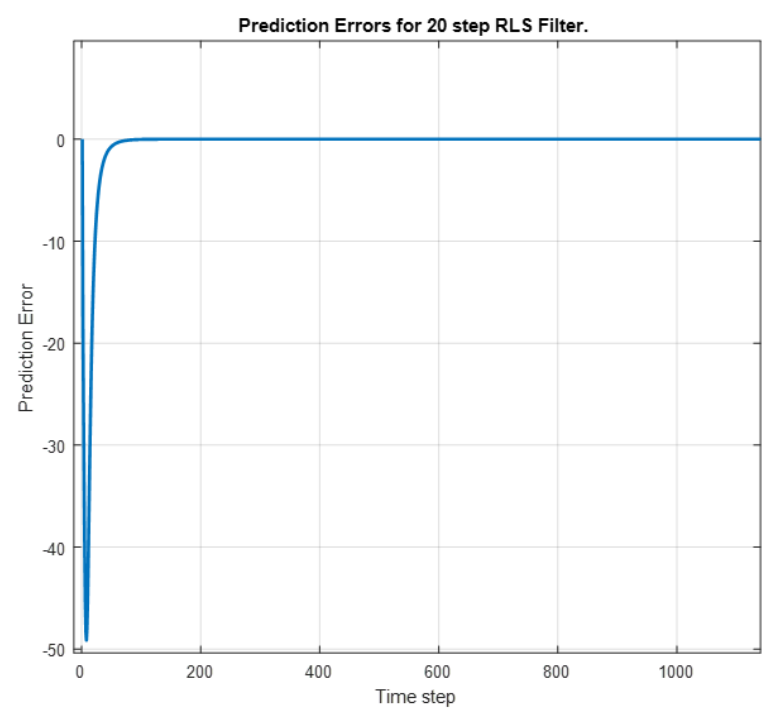

Figure 3.25: Prediction Errors of the 20 step RLS Filter.

Once again we observe that the prediction errors of the 20 step RLS filter (i.e $d_{i}=20$ ) are close to zero, indicating that the coordinates of the 20 step RLS filter match correctly with the input command. We can conclude that the delay is identified to be 20 time steps which is 2 seconds.

Comparing $x_{w p}$ and $y_{w p}$ of the four RLS filters we get, 


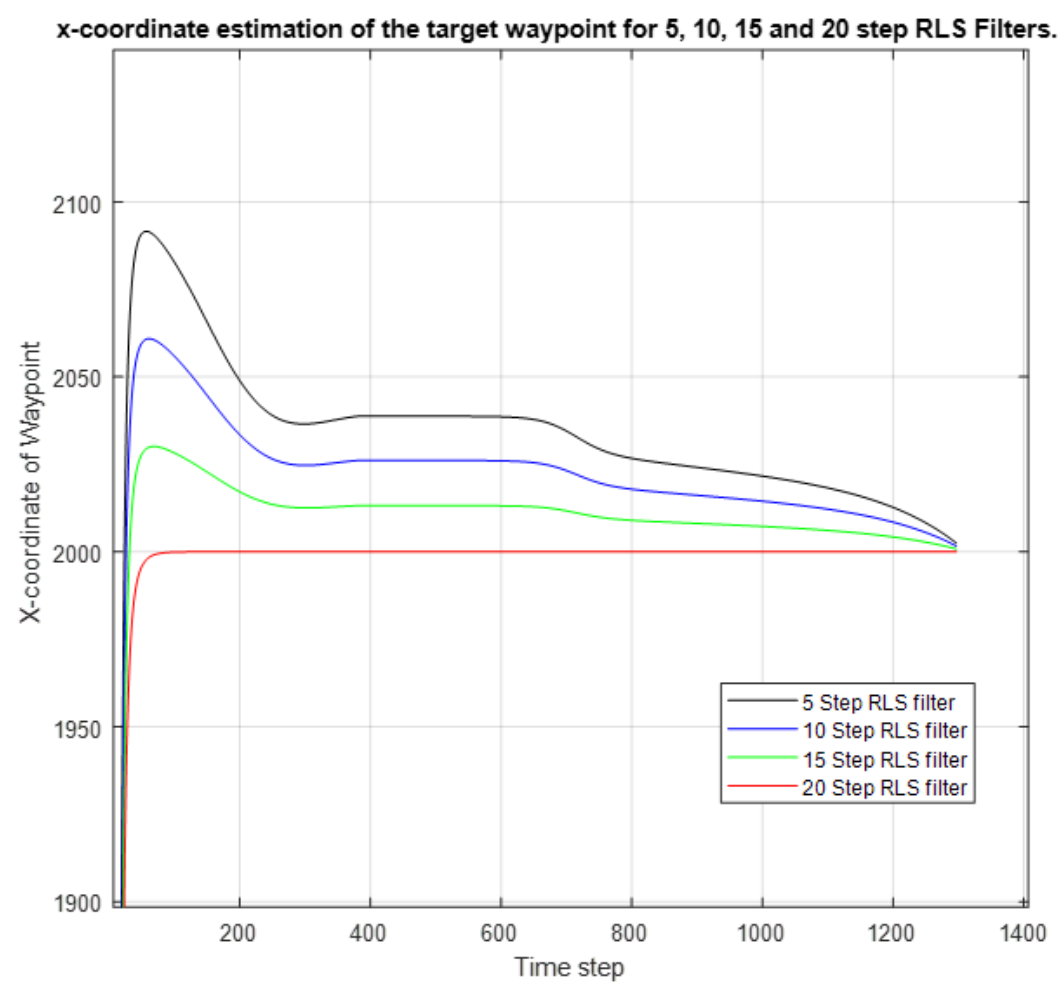

Figure 3.26: $x_{w p}$ RLS estimate of the target waypoint. The black line shows the estimate of the 5 step LS filter, the blue line shows the estimate of the 10 step LS filter, the green line shows the estimate of the 15 step LS filter and the red line shows the estimate of the 20 step LS filter. 


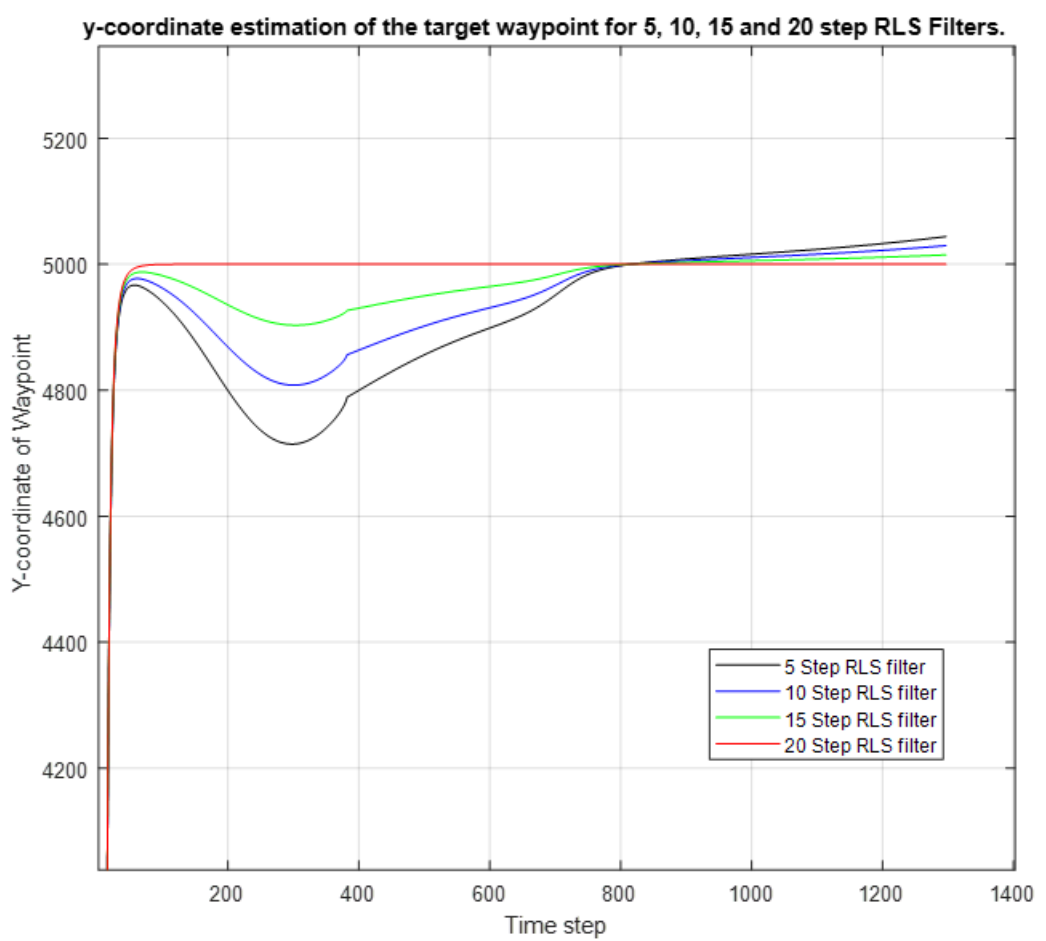

Figure 3.27: $y_{w p}$ RLS estimate of the target waypoint. The black line shows the estimate of the 5 step LS filter, the blue line shows the estimate of the 10 step LS filter, the green line shows the estimate of the 15 step LS filter and the red line shows the estimate of the 20 step LS filter.

Comparing the prediction errors of the four filters in figure 3.28, we note the differences in the errors and we can see that the prediction errors of the 20 step RLS filter converges to zero. For clarity figure 3.29 shows the prediction errors from 0 to 700 time steps and figure 3.30 shows the prediction errors from 700 to 1100 time steps. 3.31 shows the prediction errors of the 20 step RLS filter, the errors are close to zero and appear as white noise. 




Figure 3.28: Prediction errors of the four RLS filters. The black line shows the errors of the 5 step RLS filter, the blue line shows the errors of the 10 step RLS filter, the green line shows the errors of the 15 step RLS filter and the red line shows the errors of the 20 step RLS filter. 


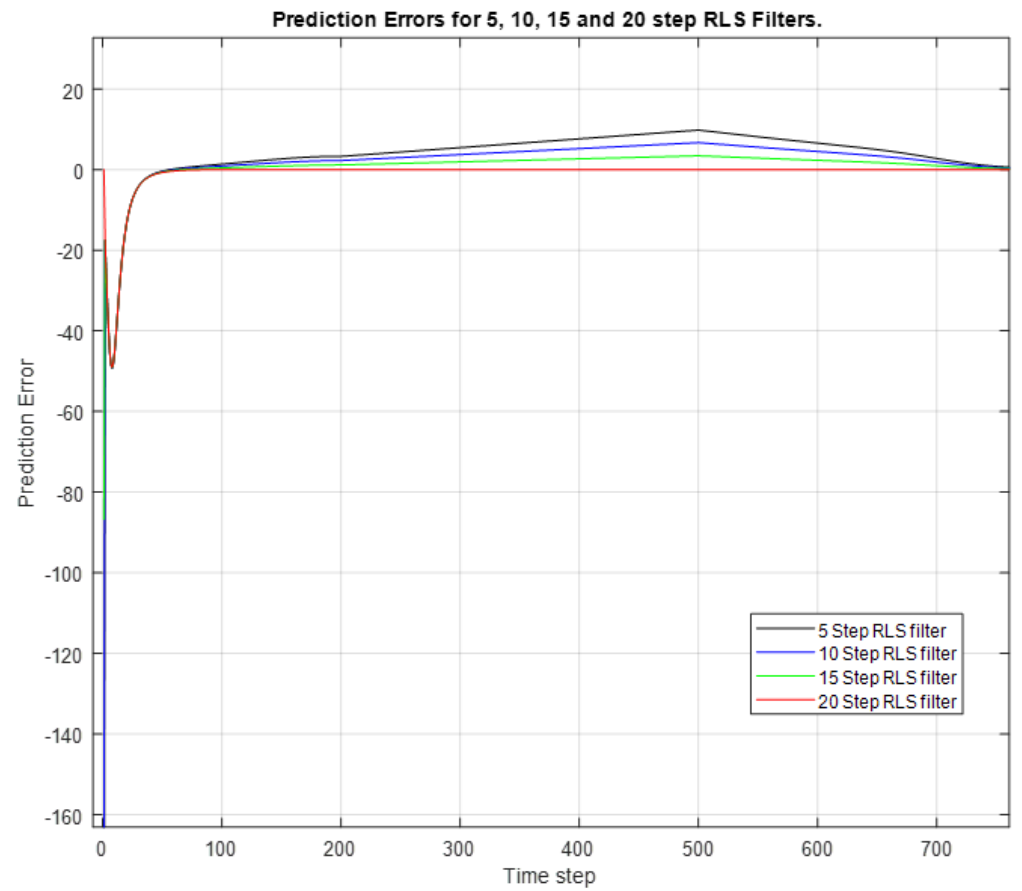

Figure 3.29: Prediction errors of the four RLS filters for 0 to 700 time steps. The black line shows the errors of the 5 step RLS filter, the blue line shows the errors of the 10 step RLS filter, the green line shows the errors of the 15 step RLS filter and the red line shows the errors of the 20 step RLS filter. 




Figure 3.30: Prediction errors of the four RLS filters for 700 to 1150 time steps. The black line shows the errors of the 5 step RLS filter, the blue line shows the errors of the 10 step RLS filter, the green line shows the errors of the 15 step RLS filter and the red line shows the errors of the 20 step RLS filter. 


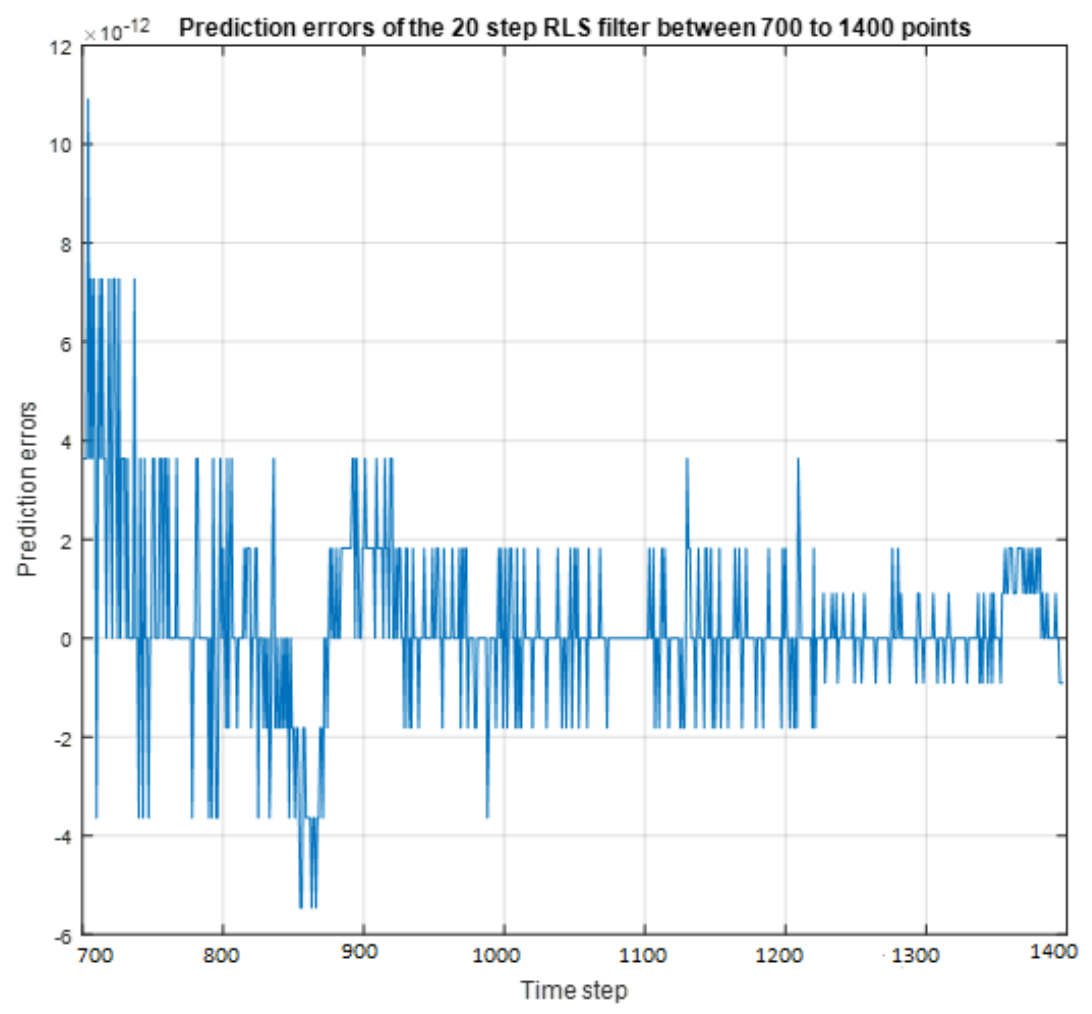

Figure 3.31: Prediction errors of the 20 step RLS filters for 700 to 1400 time steps. The errors are close to zero and appear as white noise.

From figure 3.28 and 3.30 we see that the prediction errors of the four filters are sometimes close to zero. We will have to define how close to zero the prediction errors should be before we can identify the filter with best waypoint estimates. Using hypothesis testing and switching, we can define how close to zero the prediction errors should be. Hypothesis testing is discussed in chapter four. 


\subsection{Delay Compensation}

Knowing the target waypoint and the delay, the UAV can autonomously compute its heading $\psi_{\text {uav }}$ to the waypoint and then navigate its way to the learnt waypoint.

$$
\psi_{u a v}(t)=\tan ^{-1}\left(\frac{\hat{\theta}_{i y}-y_{u a v}(t)}{\hat{\theta}_{i x}-x_{u a v}(t)}\right)
$$

Where $\hat{\theta}_{i x}$ and $\hat{\theta}_{i y}$ are the xy-coordinates of the target waypoint obtained from the selected $i$ th filter that determines the delay and waypoint. The position $x_{u a v}(t)$ and $y_{\text {uav }}(t)$ are the $\mathrm{x}-\mathrm{y}$ coordinates of the UAVs location at time $t$. While the UAV executes its self generated command $\psi_{\text {uav }}(t)$, the UAV ignores the delayed commands of the teleoperator and keeps track of the teleoperator commands so that when there is a change in either the delay or the waypoint, there is a corresponding adjustment. Figure 3.32 shows how a $10 \mathrm{~s}$ delay input command is compensated for when the delay and waypoints are learnt. In this case, a 10 step RLS filter is selected, therefore $\hat{\theta}_{i x}=\hat{\theta}_{10 x}$ and $\hat{\theta}_{i y}=\hat{\theta}_{10 y}$. 


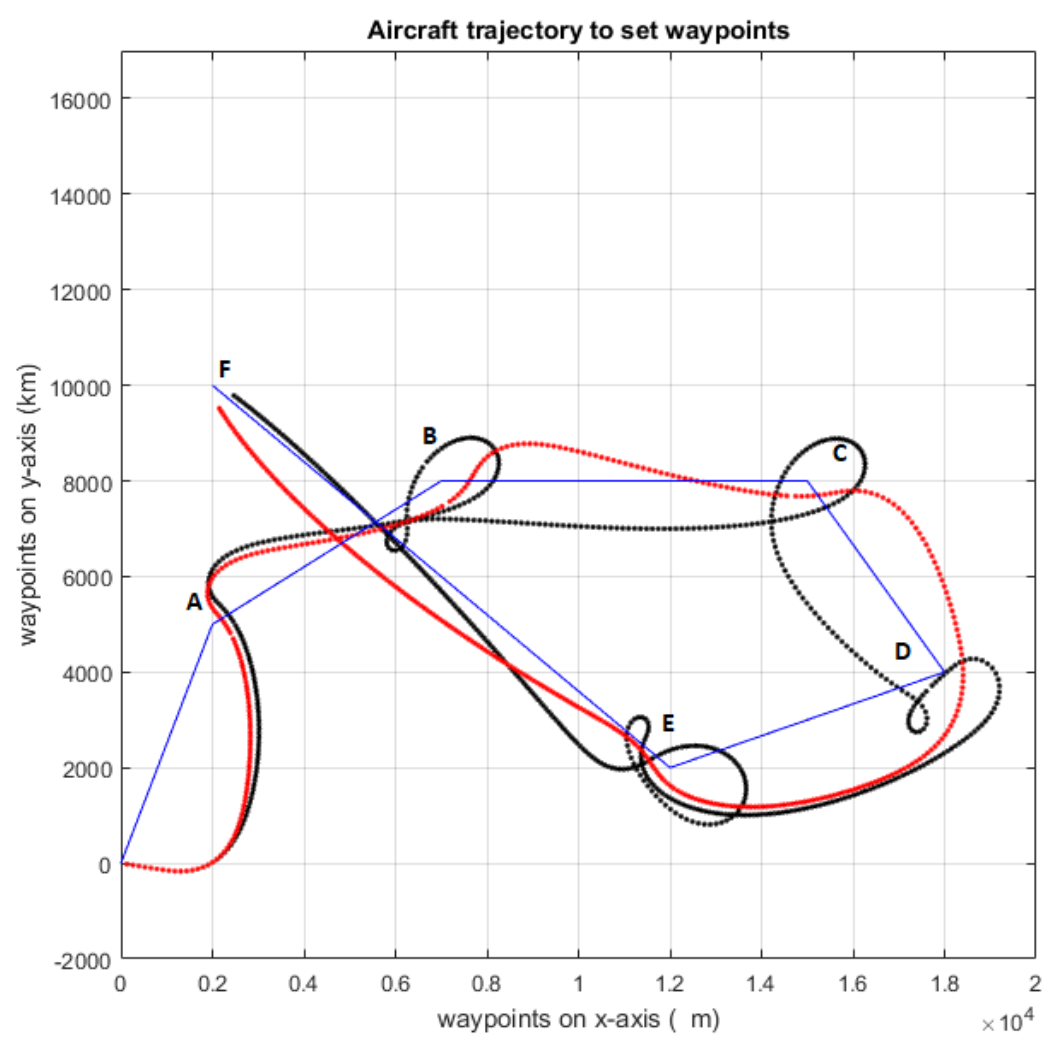

Figure 3.32: The UAV trajectory when a $10 s$ delay in input commands is being compensated for by autonavigation. The black dotted line indicates the aircraft trajectory without compensation, the blue line is the straight line between waypoints and the red dotted line indicates the UAV trajectory with compensation.

We see that in the trajectory of the UAV receiving delayed input commands (black line), the UAV misses the target waypoints. In the trajectory of the UAV executing autonomosly generated commands (red line), there are no target misses. We also observe that there is a greater displacement between the trajectory of delayed commands (black line) and the line between waypoints (blue line) compared to the displacement between trajectory of autonomous navigation (red line) and the line between waypoints (blue line).

In the next chapter, we discuss a method for filter selection and how switching from teleoperator to autonavigation is performed. 


\section{Chapter 4}

\section{Hypothesis testing and Delay \\ Compensation}

\subsection{Introduction to Hypothesis Testing for Filter Selection}

Hypothesis testing is a useful method for model selection [19] 20] 21. In chapter 3, we designed four RLS filters to identify delays in input command to a UAV controller. The criteria for filter selection was to select the RLS filter with close to zero prediction errors. Each RLS filter $i$ tracks past UAV coordinates in a specified time step $d_{i}$. For example a 5 step RLS filter tracks UAV coordinates 5 steps back. To learn the target waypoint and delay, the received input commands are matched with the coordinates of the filters. The equation of line passing through each coordinate is defined by

$$
-m(t) x_{w p}+y_{w p}=-m(t) x_{u a v}(t)+y_{u a v}(t)
$$

where $-m(t)=\tan \psi_{\text {command }}(t)$. The filter with a close to zero prediction error, determines the waypoint and the delay. If a 5 step RLS filter gives a close to zero 
prediction error then the delay is identified as 5 time steps which is equivalent to 0.5 seconds and the waypoint is the estimate of the 5 step RLS filter, i.e $\hat{\Theta}_{5}=\left[\theta_{5 x}, \theta_{5 y}\right]$ where $\theta_{5 x}, \theta_{5 y}$ are the $x$ and $y$ coordinates of the estimated waypoint. From figure 3.28 in chapter 3 , we observed that the prediction errors of the four filters are sometimes close to zero and because there can not be more than one delay at the same time, we will have to design a metric by which we define how close to zero the prediction errors should be for filter selection.

Hypothesis testing compares statistical data sets, obtained from experiments, with an ideal model. From this comparison, one can then define a relationship between the obtained data set and the ideal model. Two hypothesis are defined 22

1. The null Hypothesis, $H_{0}$ : This is the default hypothesis, the currently accepted value for a parameter.

2. The alternate Hypothesis, $H_{1}$ : This is the claim that $H_{0}$ is false.

Hypothesis testing determines what outcomes lead to the rejection or acceptance of the null hypothesis for a specified level of significance $\alpha$. The level of significance $\alpha$, is the probability of rejecting the null hypothesis given that it is true. In this thesis an $n$-sample hypothesis test method is proposed for filter selection. When the hypothesis test detects a RLS filter with the accurate delay estimate, the filter becomes selected.

When the prediction errors of a RLS filter are sufficiently close to zero, this indicates that the input commands match accurately with the past UAV coordinates tracked by the RLS filter and the estimate $\hat{\Theta}$ is an estimation of the waypoint as discussed in chapter three. In the event that there is a mismatch between the input commands and the coordinates the RLS filter tracks, there will be an increase in the absolute value of the prediction errors. The change in statistic of the prediction 
errors can be observed using a t-test. The t-test is a statistical hypothesis test that compares two averages (means) and tells us if they are different from each other 23 . Usually one mean is obtained from the experimental samples and the other is the mean of an ideal model. It is assumed that the experiment data follows a normal distribution. For example, suppose there is a claim that a particular item takes a mean of 22 minutes to produce. In an effort to test this claim, 50 of the same item were produced and the mean production time was found to be 25.36 minutes with a standard deviation of 7.24 minutes. Using a one sample t-test, we want to test the claim at a 0.1 level of significance that the production time of the item is 22 minutes. In this example, we are comparing the experimental mean $(\bar{x}=25.36$ minutes) to an ideal mean ( $\bar{\mu}=22$ minutes) to see if they are statistically equal. A test for equality indicates a two-tailed test. First we define the hypothesis:

1. $H_{0}$ (null Hypothesis): $\bar{\mu}=22$ minutes.

2. $H_{1}$ (alternate Hypothesis): $\bar{\mu} \neq 22$ minutes.

State the level of significance $\alpha$,

$$
\alpha=0.1
$$

Define the t-test statistic

$$
t_{\text {stat }}=\frac{\bar{x}-\bar{\mu}}{S / \sqrt{n}}
$$

where $\bar{x}$ is the sample mean (experiment mean), $\bar{\mu}$ is the ideal mean, $S$ is the sample standard deviation and $n$ is the sample size.

$$
t_{\text {stat }}=\frac{25.36-22}{7.24 / \sqrt{50}}=3.28
$$

we now check the statistical $t$-tables for the critical value at 0.1 level of significance. The critical value is a point on the $t$-distribution that determines whether or not to reject the hypothesis. Since this is a two tailed test, a 0.1 significance level is 
equivalent to a 0.05 significance level in each tail. The critical value $t$ at a $\alpha=0.05$ level of significance with the degree of freedom $n-1=49$ is 1.677. Figure 4.1 illustrates a two tailed t-test for this example.

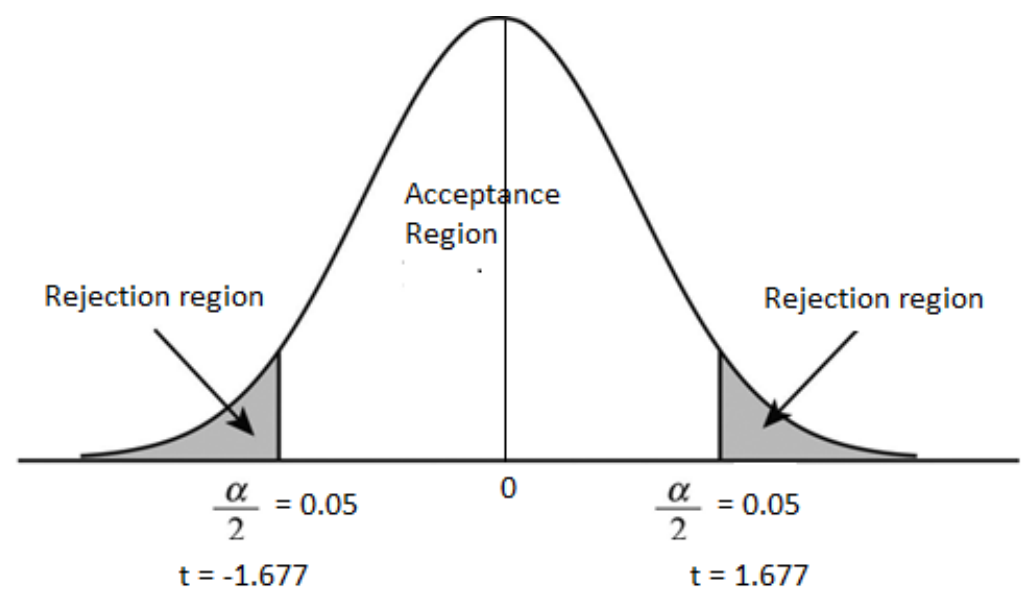

Figure 4.1: Two tailed t-test at a $\alpha$ level of significance.

For any value of the test statistic $t_{\text {stat }}$ that falls between the range of values from -1.677 to +1.677 (acceptance region) as illustrated in figure 4.1, the null hypothesis is accepted. If the value of $t_{\text {stat }}$ falls outside of the range from -1.677 to +1.677 (rejection region), the null hypothesis is rejected. In the example, $t_{\text {stat }}=3.28$ is greater than the critical value on the right tail $t=1.677$ (i.e it falls outside of the range), hence $t_{\text {stat }}=3.28$ is in the rejection region. We therefore reject the null hypothesis $H_{0}$ at a 0.1 level of significance. This would suggest that the mean production time of the item is not 22 minutes.

Similarly, if we define an hypothesis that the mean prediction errors of a RLS filter whose coordinates match accurately with input command equals zero and then test the hypothesis on each RLS filter for $n$ sample of prediction errors, we can accept/reject (select/deselect) a RLS filter whose t-statistic falls in a acceptance/rejection region. In this case, the ideal mean $\bar{\mu}=0$ and the experimental 
mean $\bar{\epsilon}$ is the mean of $n$ samples of past prediction errors of the RLS filter. This defines how close to zero the prediction errors of the RLS filter should be for selection. Lets define the test statistic $t_{i_{s t a t}}(t)$ for the $i$ th RLS filter as:

$$
t_{i_{s t a t}}(t)=\frac{\bar{\epsilon}_{i}(t)-\bar{\mu}}{S(t) / \sqrt{n}}
$$

where $n$ is the sample size of past observed prediction errors, $\bar{\mu}$ is the ideal mean of prediction errors $(\bar{\mu}=0)$ and $\bar{\epsilon}_{i}(t)$ is the mean of $n$ samples of past prediction errors of the $i$ th RLS filter. $S_{i}(t)$ is the running standard deviation of the $i$ th RLS filter and is calculated as

$$
S^{2}(t)=\frac{\sum_{t=1}^{n}\left(\epsilon_{i}(t)-\bar{\epsilon}_{i}\right)^{2}}{n-1}
$$

$\bar{\epsilon}_{i}$ is calculated as:

$$
\bar{\epsilon}_{i}=\frac{\sum_{t=1}^{n} \epsilon_{i}(t)}{n}
$$

A binary decision rule is used to select or deselect the RLS filter whose statistic accepts or rejects the null hypothesis within a user-defined rejection threshold, denoted by $\operatorname{Tr}$. $\operatorname{Tr}$ corresponds to the critical value from statistical t-tables for a specified level of significance $\alpha$. If a RLS filter accepts the null hypothesis, the filter is selected. If a filter rejects the null hypothesis, the filter is deselected. The hypotheses are defined as:

1. The null hypothesis $H_{0}:$ if $-T_{r} \leqslant t_{\text {stat }} \leqslant T_{r}$, Accept $H_{0}$. This means that the prediction errors of the given filter are close to zero and the test statistic falls within the acceptance region and as discussed in chapter three, this RLS filter determines the waypoint and delay. Therefore select this RLS filter.

2. The alternate hypothesis $H_{1}$ : if $t_{\text {stat }}<-T_{r}$ or $t_{\text {stat }}>T_{r}$ : Reject $H_{0}$. This suggests that the prediction errors of the given filter are not close to zero and that the test statistic falls outside the acceptance region, as such this RLS 
filter has not determined the waypoint and delay. The given RLS filter is not selected.

Figure 4.2 illustrates the hypothesis test accept/reject criteria.

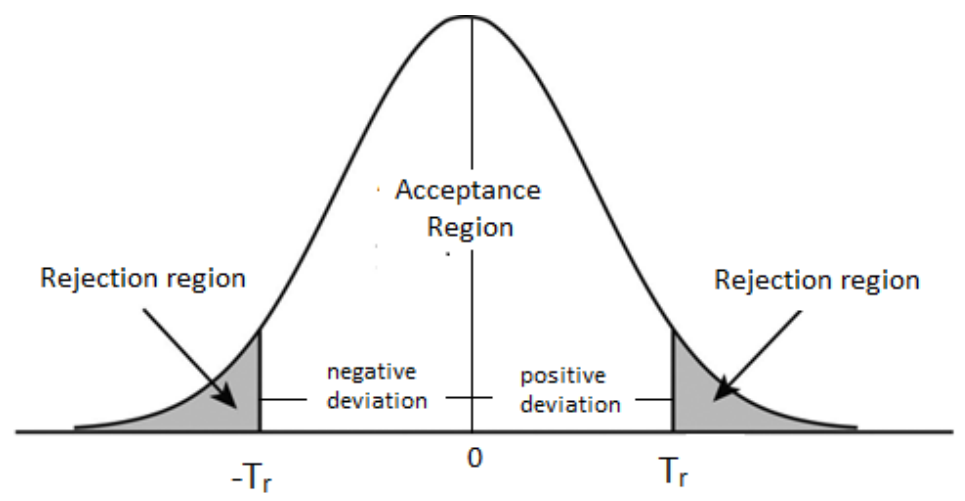

Figure 4.2: Two tailed t-test within a user-defined rejection threshold $T r$.

Traditionally, eqs. 4.2 and 4.3 are used when the experiment data follows a normal distribution. However, the prediction errors $\epsilon(t)$, are not normally distributed. Hence we propose that 4.3 be modified using the variance and standard deviation of all the prediction errors of the filters. The running standard deviation is modified as:

$$
S_{d}^{2}(t)=\frac{\sum_{i=1}^{N}\left(\sum_{j=1}^{n}\left(\bar{E}(t)-\epsilon_{i j}(t)\right)^{2}\right)}{(n \times N)-1}
$$

where $\epsilon_{i j}$ is the $j$ th prediction error of the $i$ th RLS filter at time $t, n$ is the sample size and $N$ is the number of RLS filters. $E(t)$ is the total sum of $n$ sample prediction errors from $N$ RLS filter at time $t$ i.e:

$$
E(t)=\sum_{i=1}^{N}\left(\sum_{j=1}^{n} \epsilon_{i j}(t)\right)
$$

$\bar{E}$ is the mean of the total sample prediction errors of $N$ RLS filters.

$$
\bar{E}(t)=\frac{E(t)}{n \times N}
$$


the test statistic for a given $i$ th RLS filter is now defined as

$$
t_{\text {stat }}(t)=\frac{\bar{\epsilon}_{i}(t)-\bar{\mu}(t)}{S_{d}(t) / \sqrt{n}}
$$

where $\bar{\epsilon}_{i}(t)$ is the mean of the last $n$ observed prediction errors of the $i$ th RLS filter at time $t$ calculated using eq $4.4 . \bar{\mu}(t)$ is the ideal mean, $S_{d}(t)$ is the running standard deviation at time $t$ and $n$ is the sample size. Basically, what we really want is to find the RLS filter with smallest mean deviation from zero (see figure 4.2).

\subsubsection{Demonstration of filter selection using Hypothesis testing}

We assume that the range of operating time delays associated with the UAV in thesis is between 0.5 seconds ( 5 time steps) to 2 seconds (20 time steps). Four RLS filters discussed in chapter 3 each matched to 5, 10, 15 and 20 time step delays are designed to cover the range of time delay associated with the UAV considered in this thesis. The hypothesis test is run on the four RLS filters to demonstrate how a filter is selected. Recall that each RLS filter structure is as defined by eq. 3.39

$$
-m(t) x_{w p}+y_{w p}=-m(t) x_{u a v}\left(t-d_{i}\right)+y_{u a v}\left(t-d_{i}\right)
$$

The RLS algorithm is defined for each filter $i$ as follows:

$$
\begin{aligned}
\Phi_{i} & =\left[\begin{array}{ll}
-m(t) & 1
\end{array}\right] \\
Y_{i} & =\left[\left(-m(t) x_{\text {uav }}\left(t-d_{i}\right)+y_{\text {uav }}\left(t-d_{i}\right)\right)\right] \\
L_{i}(t) & =\frac{P_{i}(t) \Phi_{i}(t)}{\lambda+\Phi_{i}^{T}(t) P_{i}(t) \Phi_{i}(t)}
\end{aligned}
$$


The prediction error for each $i$ th RLS filter, which is the experiment data whose mean we are comparing to an ideal mean $\mu=0$ is

$$
\epsilon_{i}(t)=\left[Y_{i}-\Phi_{i}^{T}(t) \hat{\Theta}_{i}(t)\right]
$$

and the waypoint estimate is

$$
\hat{\Theta}_{i}(t+1)=\hat{\Theta}_{i}(t)+L_{i}(t) \epsilon_{i}(t)
$$

where $m=\tan \psi_{\text {command }}(t)$.

Three cases of time delay will be investigated. The first case investigates how filter selection is performed when there is a uniform delay. The second case investigates how a filter is selected when the delay changes. The third case investigates how and what filter gets selected when the true delay is not matched to the bank of RLS filters. The simulation is done in MATLAB and each time step is 0.1 seconds. The rejection/acceptance threshold value is selected as $T_{r}=1.3$ which corresponds to a significance level $\alpha$ of 0.2 (i.e 0.1 significance level in each tail) on the statistical t-tables at 19 degrees of freedom ( degree of freedom $=n-1$ ). The hypothesis we are testing is

1. The null hypothesis $H_{0}$ : if $-1.3 \leqslant t_{\text {stat }} \leqslant 1.3$, accept $H_{0}$. The RLS filter $i$ with test statistic $t_{\text {stat }}$ that falls in the range between -1.3 and 1.3 indicates the RLS filter whose mean prediction error is small and close to zero. This RLS filter determines the delay and the target waypoint. This RLS filter is therefore selected.

2. The alternate hypothesis $H_{1}$ : if $t_{\text {stat }}<-1.3$ or $t_{\text {stat }}>1.3$ : Reject $H_{0}$. The RLS filter $i$ with test statistic $t_{\text {stat }}$ that falls outside of the range between -1.3 
and 1.3 indicates the RLS filter whose mean prediction error is large or not close to zero as such the given RLS filter is not selected.

\section{Case 1: When the input command is subjected to uniform timed delay}

This case demonstrates what filter gets selected when the input commands are subjected to constant timed delays. We run four different simulations, one each for 0.5 seconds delay (5 time steps), 1 second delay (10 time steps), 1.5 seconds delay (15 time steps) and 2 seconds delay(20 time steps). Figures 4.3, 4.5, 4.7 and 4.9 illustrates the results from the hypothesis test and what filter is selected in each simulation.
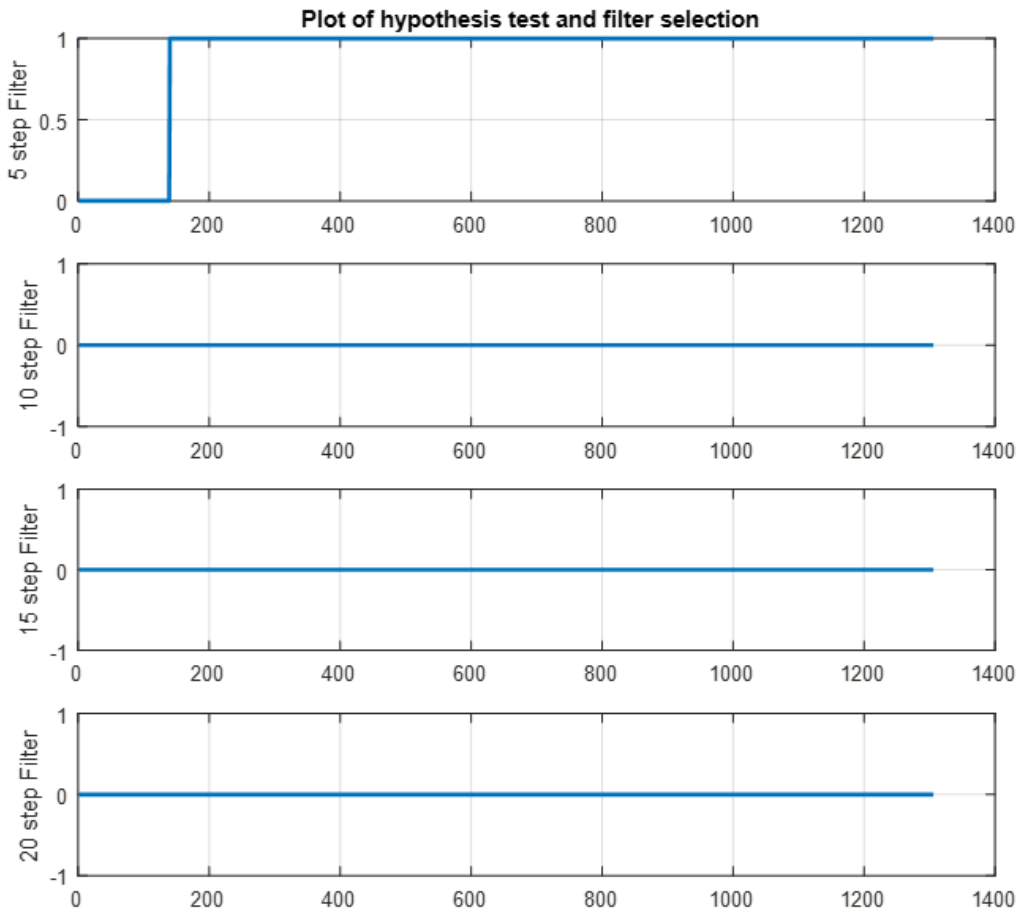

Figure 4.3: Hypothesis test selection of 5 step RLS filter. 1 indicates that the corresponding RLS filter is selected. 0 indicates that the corresponding RLS filter is not selected.

In the first simulation, the input command is subjected to a constant time delay of 
0.5 seconds (5 time steps). At approximately 140 time steps which is approximately 14 seconds, the test statistic $t_{\text {stat }}(t)$ of the 5 step RLS filter falls within the range between -1.3 and 1.3. The null hypothesis is accepted and the 5 step RLS filter is selected as illustrated in figure 4.3 . Consequently, the delay is selected as 5 time steps which is 0.5 seconds and the waypoint coordinate is selected as $\hat{\Theta}_{5}(t)$ estimate (i.e $\left.\left[\hat{\theta}_{5(x)}(t)=2000, \hat{\theta}_{5(y)}(t)=5000\right]\right)$. The test statistic $t_{\text {stat }}$ of the 10,15 and 20 step RLS filters fall outside the range between -1.3 and 1.3, the null hypothesis is rejected and the filters are not selected. The test statistic for the four RLS filters with a constant 0.5 seconds ( 5 time steps) is illustrated in figure 4.4

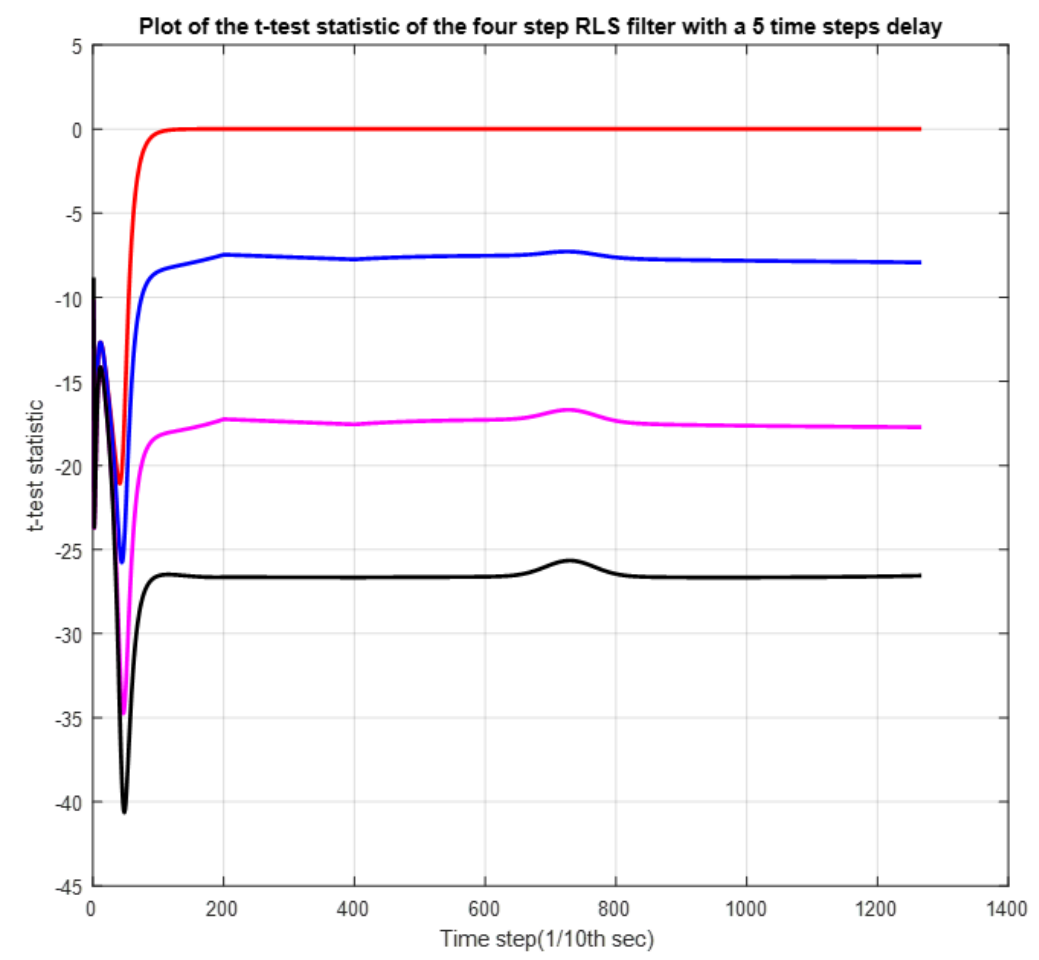

Figure 4.4: T-test statistic of 5 (red), 10 (blue), 15 (magenta) and 20 (black) step RLS filters.

In the second simulation, the input command is subjected to a constant time delay of 1 second (10 time steps). Similar to the first simulation with 5 time steps, at approximately 140 time steps which is approximately 14 seconds, the test statistic $t_{\text {stat }}(t)$ of 
the 10 step RLS filter falls within the range between -1.3 and 1.3. The null hypothesis is accepted and the 10 step RLS filter is selected as illustrated in figure 4.5. Consequently, the delay is selected as 10 time steps which is 1 second and the waypoint coordinate is selected as $\hat{\Theta}_{10}(t)$ estimate $\left(\right.$ i.e $\left.\left[\hat{\theta}_{10(x)}(t)=2000, \hat{\theta}_{10(y)}(t)=5000\right]\right)$. The test statistic $t_{\text {stat }}$ of the 5, 15 and 20 step RLS filters fall outside the range between -1.3 and 1.3 , the null hypothesis is rejected and the filters are not selected. The test statistic for the four RLS filters with a constant 1 second (10 time steps) is illustrated in figure 4.6 .
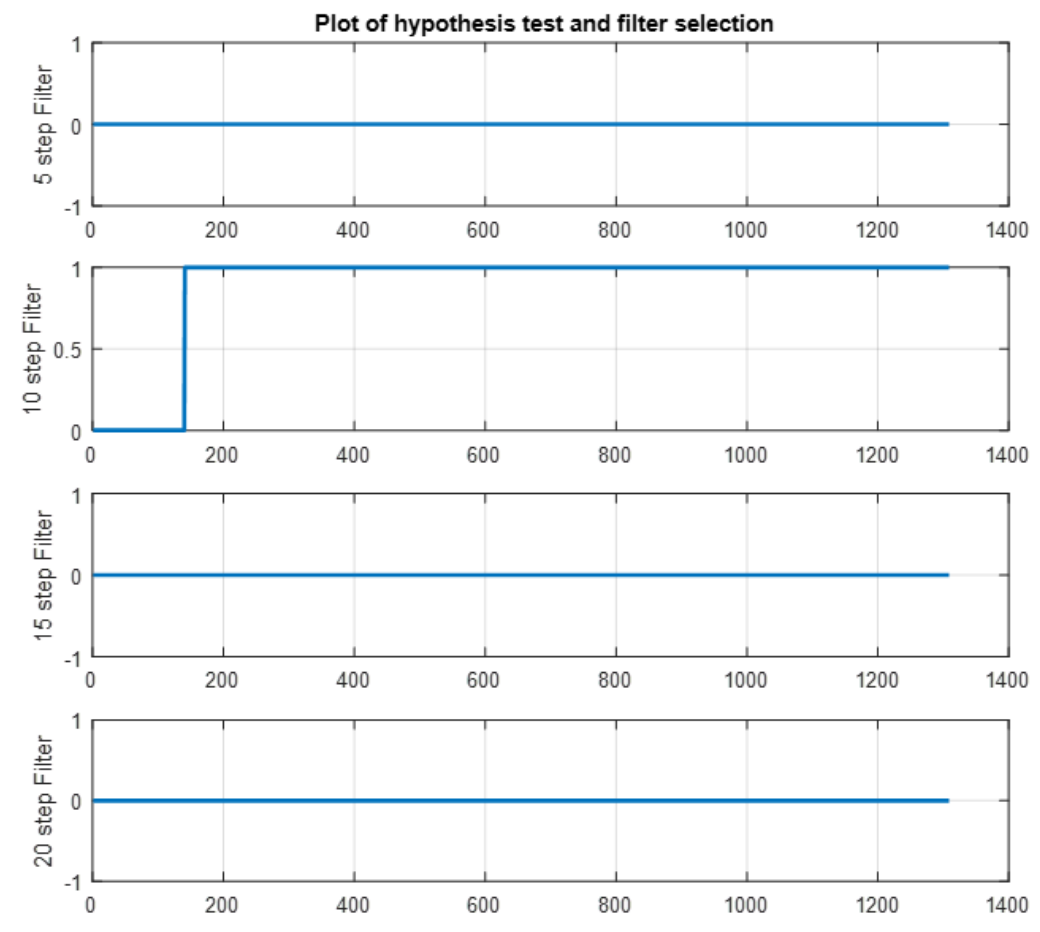

Figure 4.5: Hypothesis test selection of 10 step RLS filter. 1 indicates that the corresponding RLS filter is selected. 0 indicates that the corresponding RLS filter is not selected. 




Figure 4.6: T-test statistic of 5 (red), 10 (blue), 15 (magenta) and 20 (black) step RLS filters.

In the third simulation, the input command is subjected to a constant time delay of 1.5 seconds (15 time steps). At approximately 142 time steps which is approximately 14.2 seconds, the test statistic $t_{\text {stat }}(t)$ of the 15 step RLS filter falls within the range between -1.3 and 1.3. The null hypothesis is accepted and the 15 step RLS filter is selected as illustrated in figure 4.7. Consequently, the delay is selected as 15 time steps which is 1.5 seconds and the waypoint coordinate is selected as $\hat{\Theta}_{15}(t)$ estimate (i.e $\left.\left[\hat{\theta}_{15(x)}(t)=2000, \hat{\theta}_{15(y)}(t)=5000\right]\right)$. The test statistic $t_{\text {stat }}$ of the 5, 10 and 20 step RLS filters fall outside the range between -1.3 and 1.3 , the null hypothesis is rejected and the filters are not selected. The test statistic for the four RLS filters with a constant 1.5 seconds (15 time steps) is illustrated in figure 4.8 . 

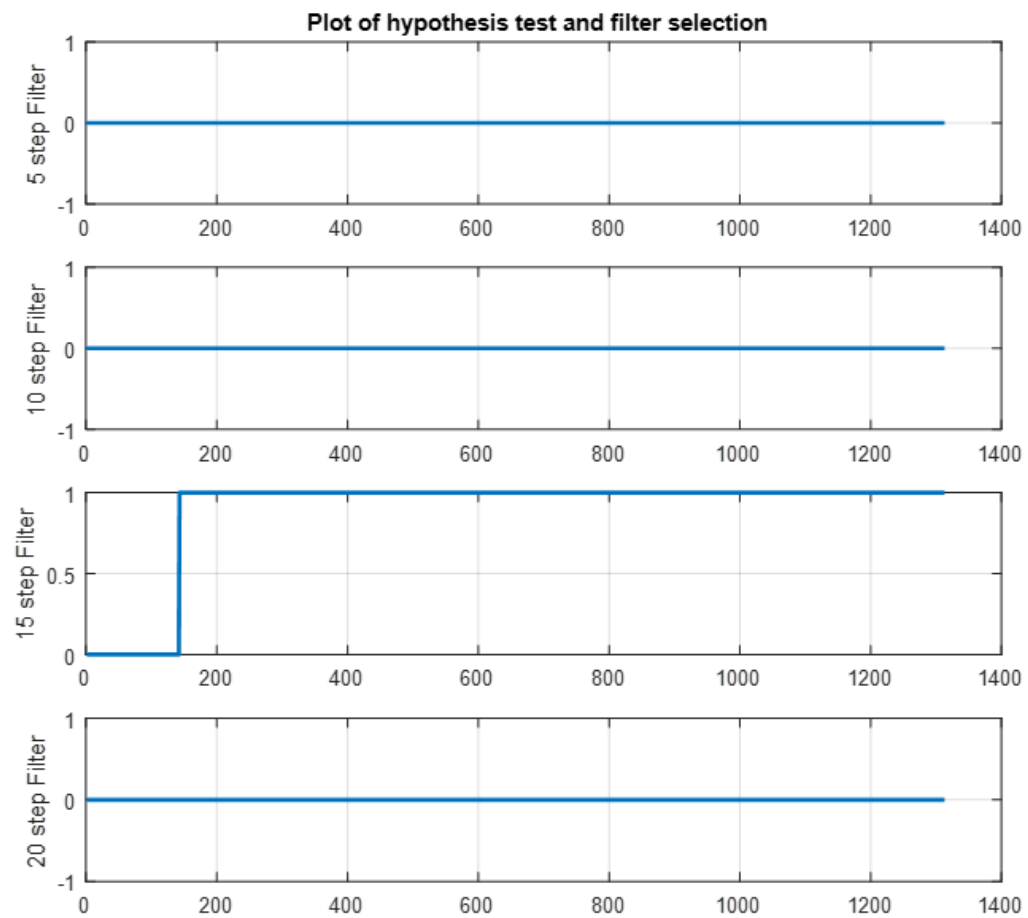

Figure 4.7: Hypothesis test selection of 15 step RLS filter. 1 indicates that the corresponding RLS filter is selected. 0 indicates that the corresponding RLS filter is not selected. 


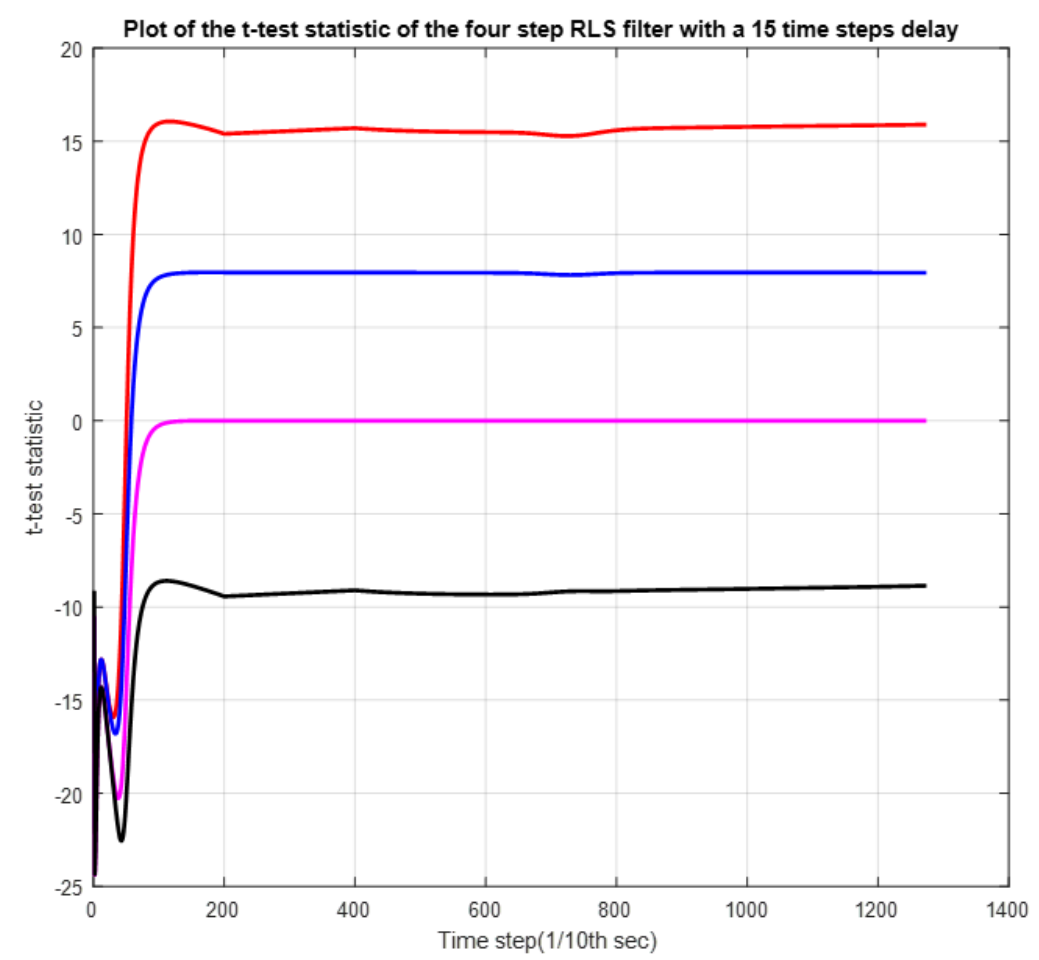

Figure 4.8: T-test statistic of 5 (red line), 10 (blue line), 15 (magenta line) and 20 (black line) step RLS filters.

In the fourth simulation, the input command is subjected to a constant time delay of 2 seconds (20 time steps). At approximately 142 time steps which is approximately 14.2 seconds, the test statistic $t_{\text {stat }}(t)$ of the 20 step RLS filter falls within the range between -1.3 and 1.3. The null hypothesis is accepted and the 20 step RLS filter is selected as illustrated in figure 4.9. Consequently, the delay is selected as 20 time steps which is 2 seconds and the waypoint coordinate is selected as $\hat{\Theta}_{20}(t)$ estimate (i.e $\left.\left[\hat{\theta}_{20(x)}(t)=2000, \hat{\theta}_{20(y)}(t)=5000\right]\right)$. The test statistic $t_{\text {stat }}$ of the 5,10 and 15 step RLS filters fall outside the range between -1.3 and 1.3 , the null hypothesis is rejected and the filters are not selected. The test statistic for the four RLS filters with a constant 2 seconds (20 time steps) is illustrated in figure 4.10 . 

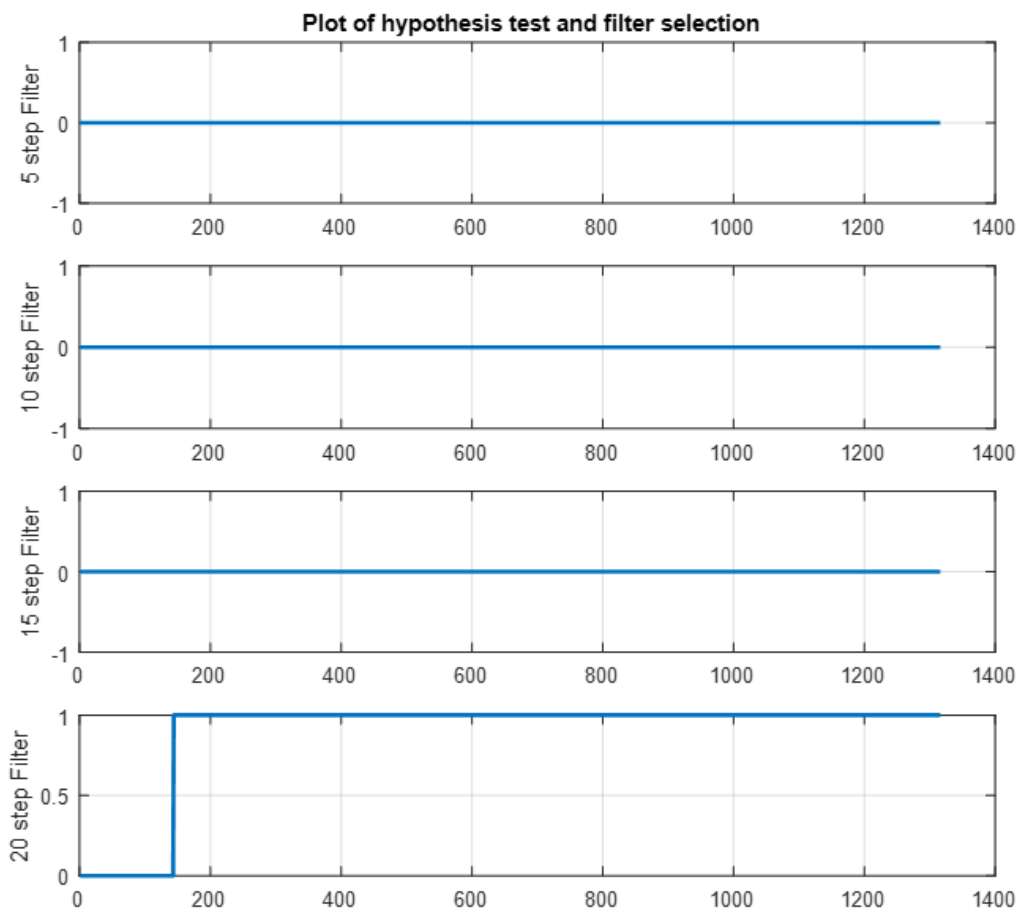

Figure 4.9: Hypothesis test selection of 20 step RLS filter. 1 indicates that the corresponding RLS filter is selected. 0 indicates that the corresponding RLS filter is not selected. 


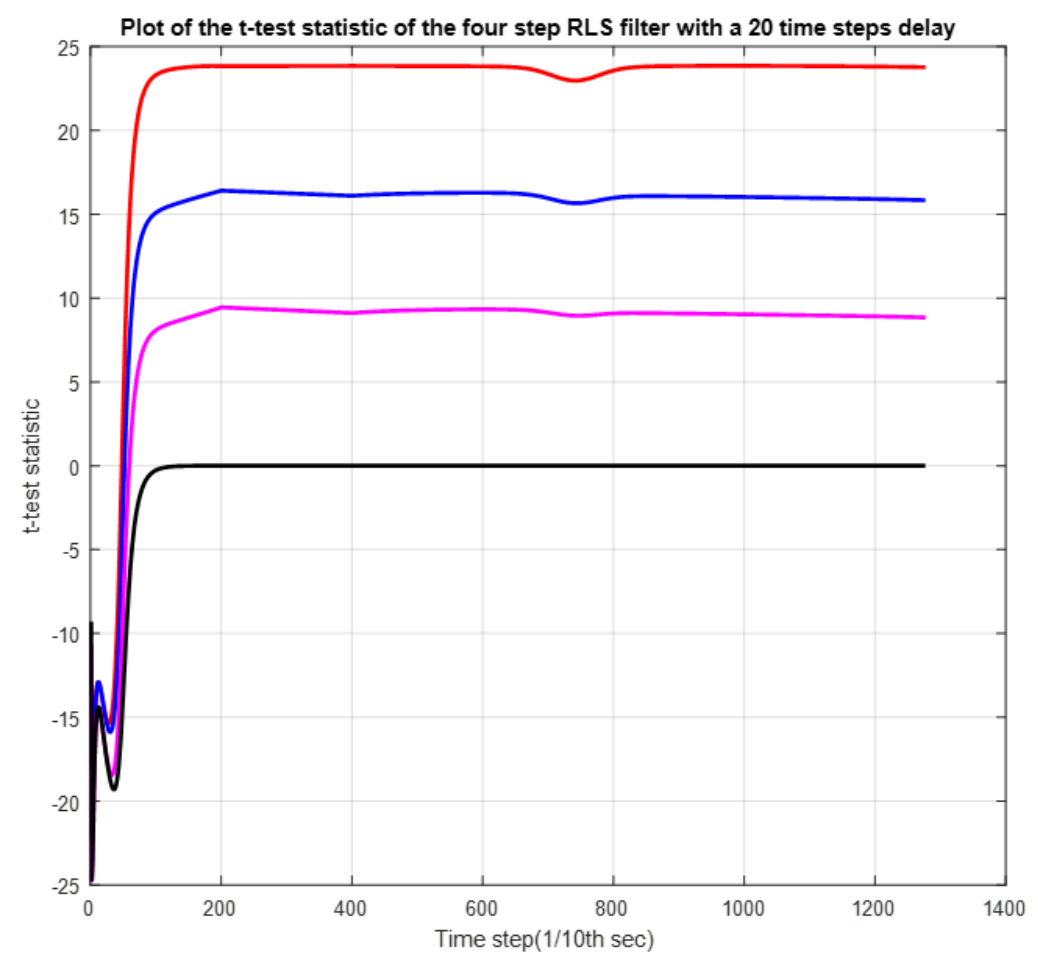

Figure 4.10: T-test statistic of 5 (red), 10 (blue), 15 (magenta) and 20 (black) step RLS filters.

Case 2: When the input command is subjected to multiple time delays.

This case demonstrates filter selection when the input commands are subjected to different time delays at different times. We run three different simulations, each for a different time delay sequence. A non-constant time delay could be as a result of changing technical, geographical and climate conditions.

In the first sequence (A), time delays are scheduled as follows: $20 \leq t \leq 400$ delay $=0.5$ second ( 5 time steps), $400<t \leq 1000$ delay $=1.5$ seconds (15 time steps) and $t>1000$ delay $=2$ seconds (20 time steps). 

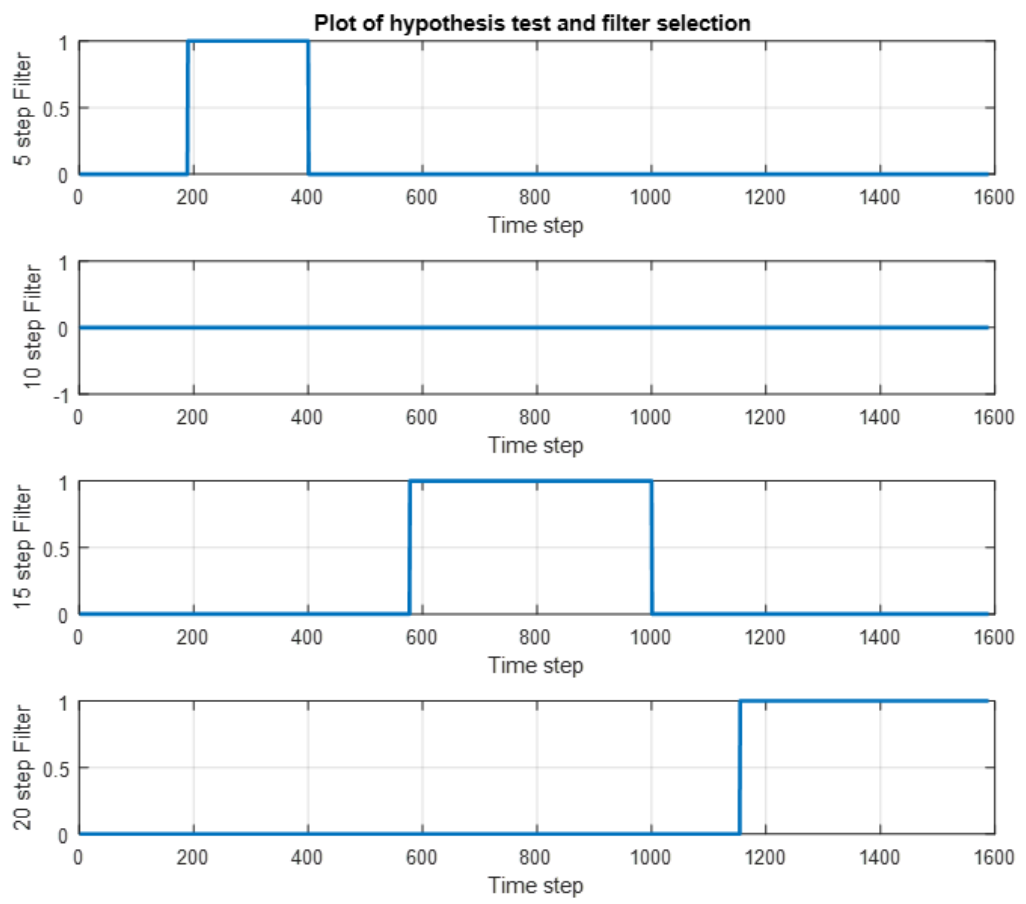

Figure 4.11: Hypothesis test selection of RLS filters in sequence A. 1 indicates that the corresponding RLS filter is selected. 0 indicates that the corresponding RLS filter is not selected.

At $t=189$ time steps $(\simeq 19$ seconds $)$ the test statistic $t_{\text {stat }}(t)$ of the 5 step RLS filter falls within the range between -1.3 and 1.3. The null hypothesis is accepted and the 5 step RLS filter is selected as illustrated in figure 4.11. The delay at this time is selected to be 5 time steps which is 0.5 seconds and the waypoint coordinate is selected as $\hat{\Theta}_{5}(t)$ estimate (i.e $\left.\left[\hat{\theta}_{5(x)}(t)=2000, \hat{\theta}_{5(y)}(t)=5000\right]\right)$. The 10,15 and 20 step RLS filters are not selected at this time because their test statistic fall outside the range of acceptance (the prediction errors are not close to zero). The 5 step RLS filter remains selected until the 400th time step as defined by the sequence, when the delay changes. This change in delay gives rise to the prediction errors causing the test statistic of the 5 RLS filter to increase beyond the acceptance region. The null hypothesis is rejected and the 5 step RLS filter is deselected. In figure 4.13, we see how the change in delay gives rise to the mean prediction errors as indicated by 
the spike in the test statistic at the 400th time step. At $t=585$ time steps $(\simeq 58.5$ seconds) the test statistic $t_{\text {stat }}(t)$ of the 15 step RLS filter falls within the range between -1.3 and 1.3. The null hypothesis is accepted and the 15 step RLS filter is selected as illustrated in figure 4.11. The 5, 10 and 20 step RLS filters are not selected at this time as their test statistics are in the rejection region. The delay at this time changes to 15 time steps which is 1.5 seconds and the waypoint coordinate is selected as $\hat{\Theta}_{15}(t)$ estimate (i.e $\left[\hat{\theta}_{15(x)}(t)=2000, \hat{\theta}_{15(y)}(t)=5000\right]$ ). The 15 step RLS filter remains selected until its test statistic fall out of the acceptance region which happens at the 1000th time step. Again we see how the change in the delay at the 1000th time step gives a rise to the mean prediction errors as indicated by the height of the curve in figure 4.13 . The difference in the heights of the curves at $t=400$ and at $t=100$ is due to the estimated solution of a larger number of data points at the 1000th time step (which is 1000 points) unlike number of data points at the 400th time step which is an estimated solution of 400 data points. Note that at $t=840$ the prediction errors go through zero. This happens because the UAV crosses the straight line between waypoints (see figure 4.12 and there is a change in the error polarity. 


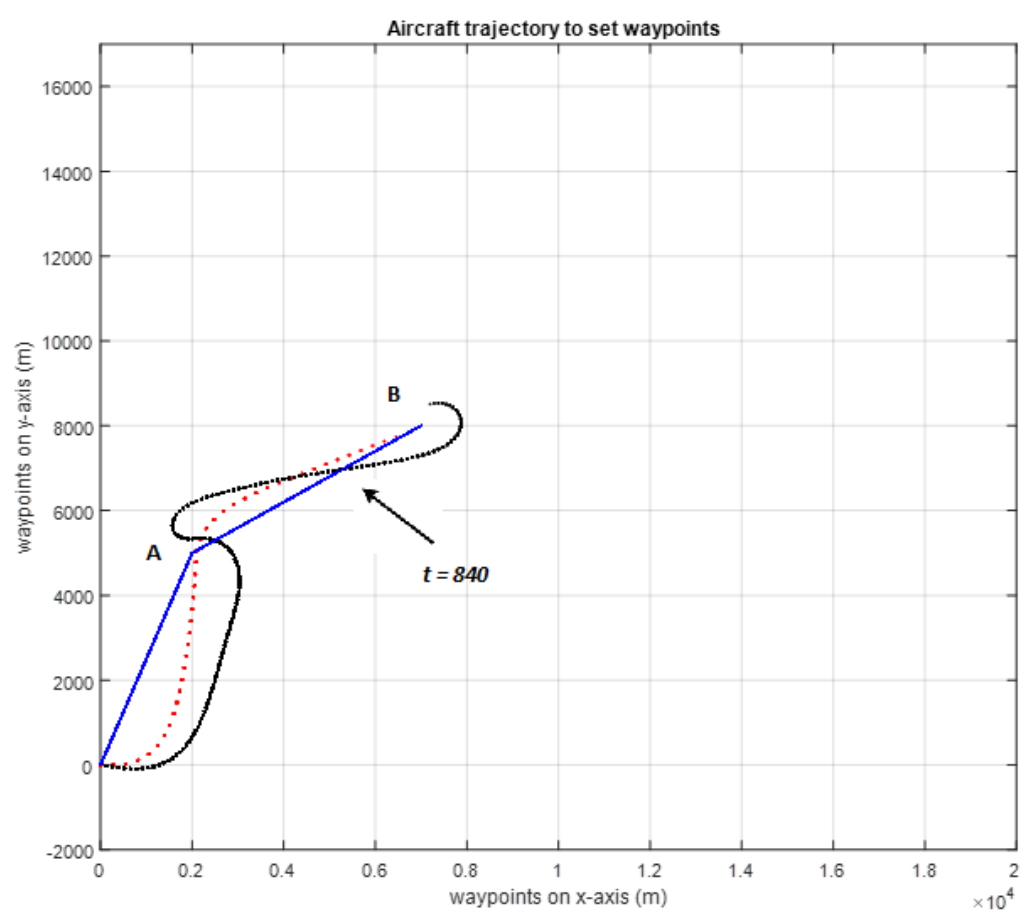

Figure 4.12: UAV trajectory when the errors go through zero at $t=840$. The red dotted line indicates the aircraft trajectory with no delay, the blue line is the straight line between waypoints and the black line indicates the UAV trajectory subjected to time delay sequence.

The filter converges to the waypoint again and at $t=1160$ time steps, the 20 step RLS filter is selected. Figure 4.11 illustrates RLS filter selections for time delay sequence A. 


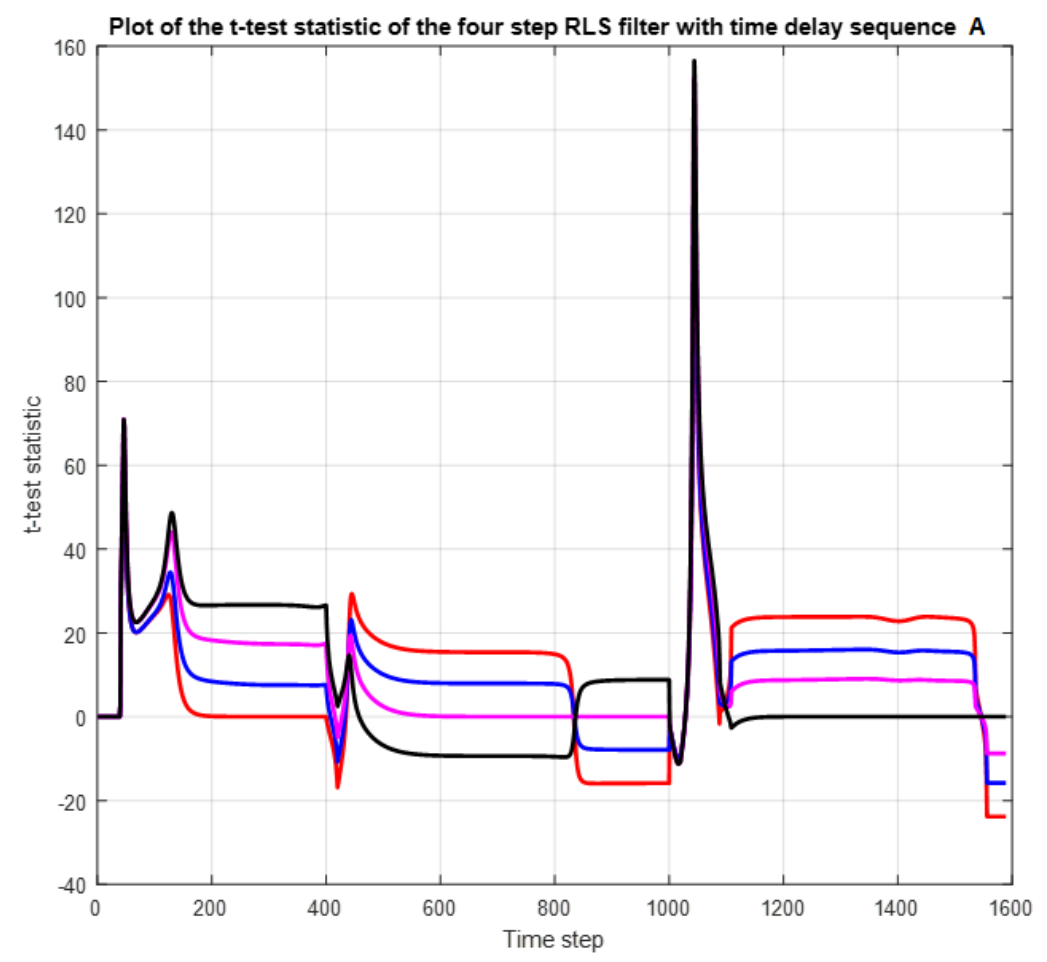

Figure 4.13: T-test statistic $t_{\text {stat }}$ of the 5 (red), 10 (blue), 15 (magenta) and 20 (black) step RLS filters in time delay sequence A

This operation is conducted in similar fashion in sequence B (figure 4.14 and sequence $\mathrm{C}$ (figure 4.16). This is to show that there is no specific preference by which the filters are selected. The filters get selected when a delay is learned and the hypothesis is accepted.

In the second sequence (B), time delays are scheduled as follows: $20 \leq t \leq 400$ delay $=2$ seconds (20 time steps), $400<t \leq 800$ delay $=5$ seconds ( 0.5 time steps) and $t>800$ delay $=1$ second (10 time steps). 

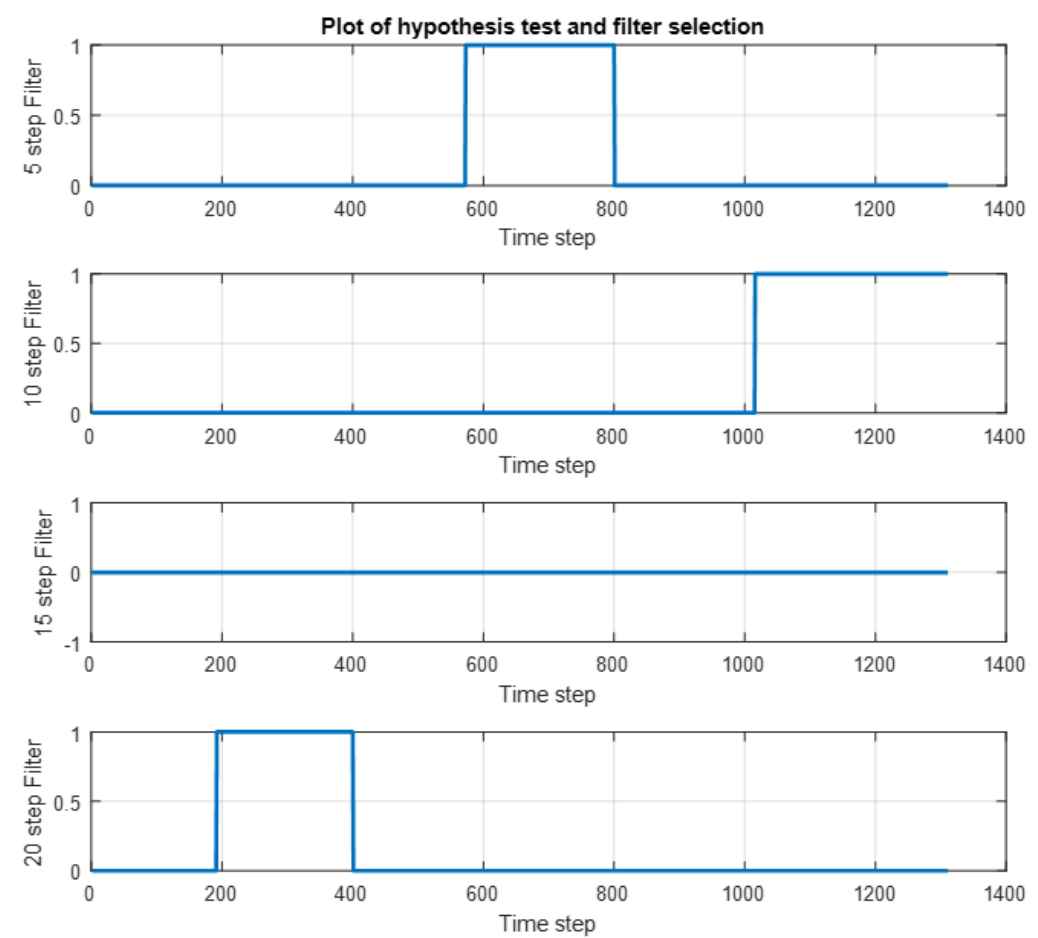

Figure 4.14: Hypothesis test selection of RLS filters in sequence B. 1 indicates that the corresponding RLS filter is selected. 0 indicates that the corresponding RLS filter is not selected.

At $t=181$, the 2 step RLS filter accepts the null hypothesis and is selected to determine the delay and the target waypoint. At $t=400$ the delay changes and the 2 step RLS filter is deselected. At $t=574$ the 5 step filter is selected. Similarly at $t=1040$ the 10 step filter is selected. We observe the change in delay at $t=400$ and $t=800$ from figure 4.15. The spikes on the test statistics of the RLS filters indicate the rise in prediction error as a result of a change in delay. 


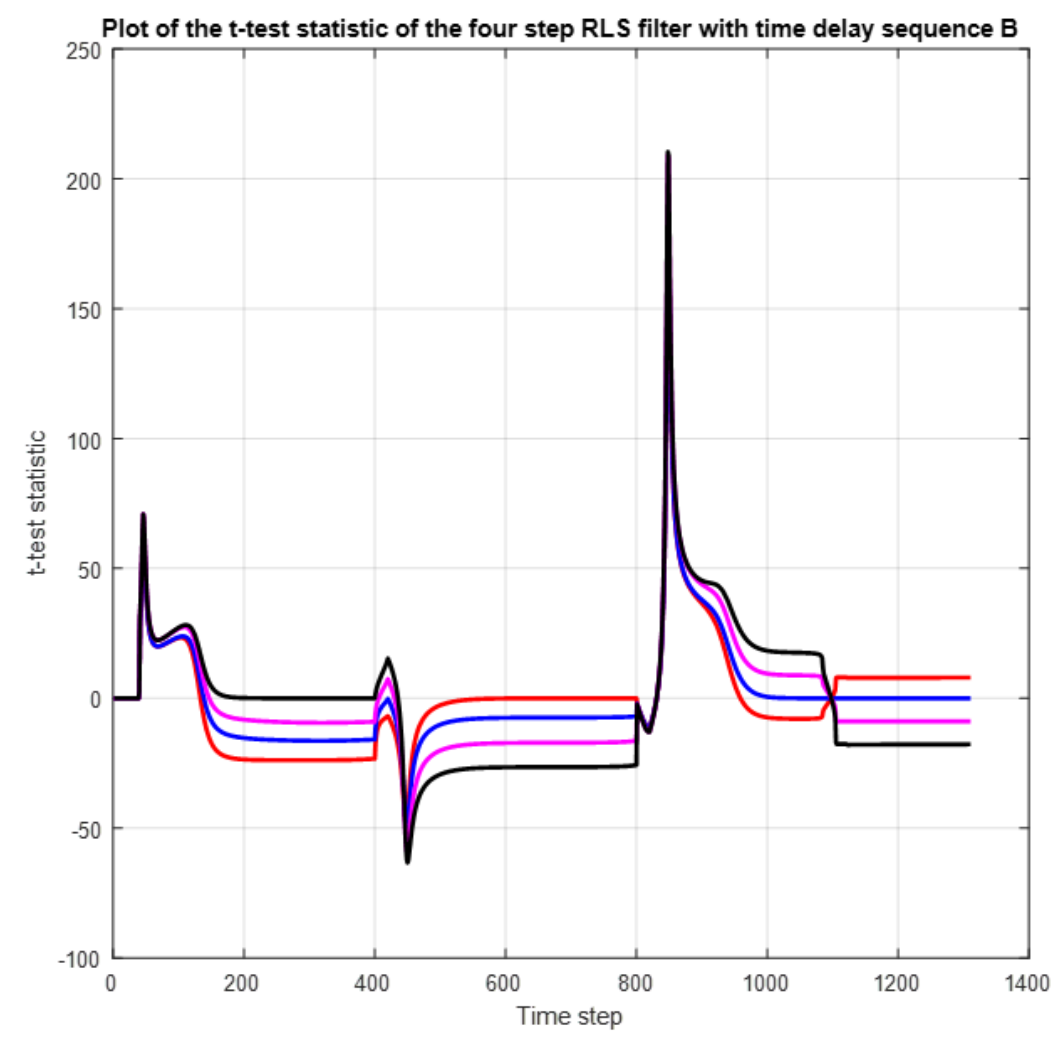

Figure 4.15: T-test statistic $t_{\text {stat }}$ of the 5 (red), 10 (blue), 15 (magenta) and 20 (black) step RLS filters in time delay sequence B

In the third sequence (C), timed delays are scheduled as follows: $20 \leq t \leq 500$ delay $=0.5$ seconds ( 5 time steps), $500<t \leq 900$ delay $=1.5$ seconds (15 time steps) and $t>900$ delay $=1$ second (10 time steps). 



Figure 4.16: Hypothesis test selection of RLS filters in sequence C. 1 indicates that the corresponding RLS filter is selected. 0 indicates that the corresponding RLS filter is not selected.

At $t=185$, the 5 step RLS filter accepts the null hypothesis and is selected to determine the delay and the target waypoint. At $t=500$ the delay changes and the 5 step RLS filter is deselected. At $t=774$ the 15 step filter is selected. Similarly at $t=1091$ the 10 step filter is selected. Again we observe the change in delay at $t=500$ and $t=900$ from figure 4.17. The spikes on the test statistics of the RLS filters indicate the rise in prediction error as a result of a change in delay. 


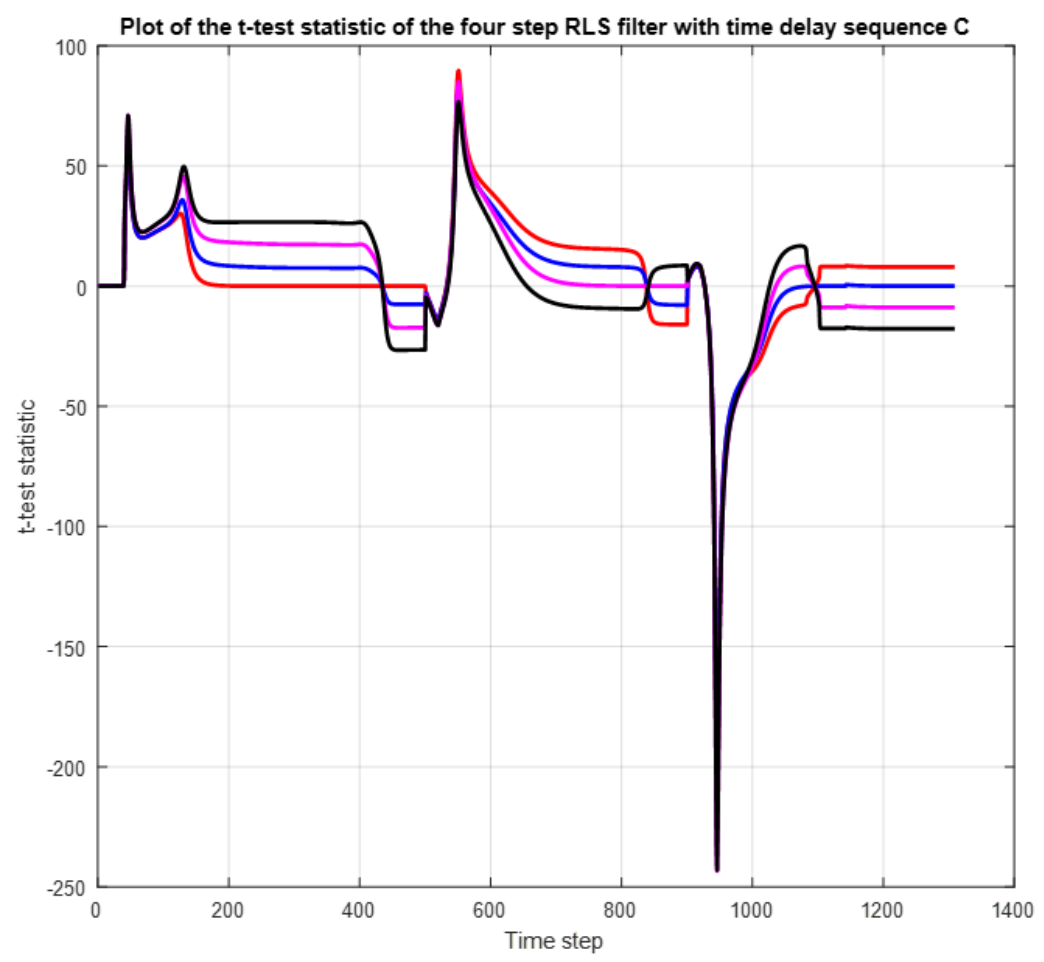

Figure 4.17: T-test statistic $t_{\text {stat }}$ of the 5 (red), 10 (blue), 15 (magenta) and 20 (black) step RLS filters in time delay sequence C

\section{Case 3: When the filters are not matched to the true time delay}

Recall, we have created a bank of RLS filters each matched to an assumed time delay of $0.5,1,1.5$ and 2 seconds. Suppose the input commands are subjected to a true time delay of 0.7 seconds ( 7 time steps), in this case the 0.7 seconds time delay does not match any of the RLS filters. In case 1 and 2, by making the accept/reject region narrow using a threshold of $T_{r}=1.3$ we are able to select the exact RLS filter that matches the true time delay. In this case where the RLS filters do not match the true delay of 0.7 seconds, we increase the accept/rejection region so that we are able to select the RLS filter with an assumed delay that is closest to the true delay (ie 0.5 second RLS filter). We select the accept/reject threshold by trial and error to cover the range of time delay that we anticipate will be experienced by 
the UAV. The accept/rejection threshold $T_{r}$ increased to 3.5 which corresponds to a significance level $\alpha$ of 0.002 (i.e 0.001 significance level in each tail). This increases the probability of selecting a RLS filter. The hypothesis we are testing is modified to

1. The null hypothesis $H_{0}$ : if $-3.5 \leqslant t_{\text {stat }} \leqslant 3.5$, accept $H_{0}$. The RLS filter $i$ with test statistic $t_{\text {stat }}$ that falls in the range between -3.5 and 3.5 indicates the RLS filter whose mean prediction error is small and close to zero. This RLS filter determines the delay and the target waypoint. This RLS filter is therefore selected.

2. The alternate hypothesis $H_{1}$ : if $t_{\text {stat }}<-3.5$ or $t_{\text {stat }}>3.5>$ : Reject $H_{0}$. The RLS filter $i$ with test statistic $t_{\text {stat }}$ that falls outside of the range between -3.5 and 3.5 indicates the RLS filter whose mean prediction error is large or not close to zero as such the given RLS filter is not selected.

For example one, we simulate with a constant timed delay of 0.7 seconds ( 7 time steps). 

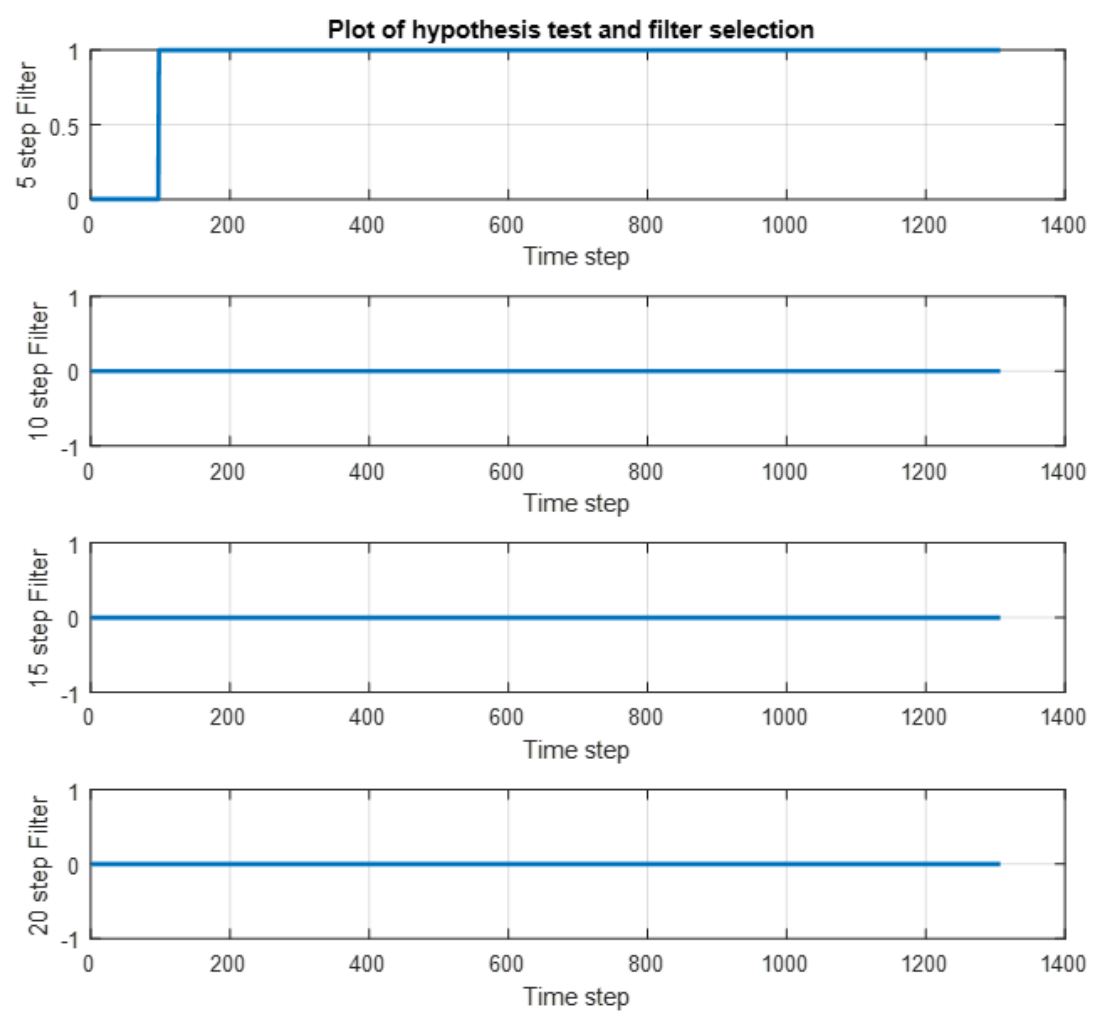

Figure 4.18: Example 1: Hypothesis test selection of 5 step RLS filter with a 0.7 timed delay. 1 indicates that the corresponding RLS filter is selected. 0 indicates that the corresponding RLS filter is not selected.

At $t=98$, approximately 10 seconds, the 5 step RLS filter accepts the hypothesis and it is selected. The delay is then approximated to be 0.5 seconds and the target waypoint is approximated to the estimate $\hat{\Theta}_{5}\left(\theta_{5 x}(t)=1998, \theta_{5 y}(t)=5012\right)$ of the 5 step RLS filter. This approximation is possible because of the selection of significance level $\alpha$ as 0.002 which increased the probability selecting a filter given that the assumed delay does not match with the actual delay. Figure 4.19 illustrates the test statistic for the four RLS filters. In a situation like this, the RLS filter that gets selected will be the RLS filter whose assumed delay is closest to the delay time step. In this example the delayed time step is 7, the RLS filter with the closest delay is the 5 step RLS filter. In some instances the test statistic of one or more RLS 
filters fall within the acceptance region, the null hypothesis is then accepted and the corresponding RLS filters are selected. This will often happen within time it takes the RLS filter to converge to a final value (figure 4.22). Another contributing factor to multiple RLS filter selection is the choice of a small significance level $\alpha$. When multiple RLS filters are selected, a second selection criteria $J$ is activated. $J$ is defined as:

$$
J(t)=\min \left(t_{i_{s t a t}}(t)\right)^{2}
$$

where $t_{i_{s t a t}}(t)$ is the test statistic of the $i$ th RLS filter at time $t . J$ emphasizes the selection of only the filter with the least mean deviation of prediction errors.



Figure 4.19: Example 1: T-test statistic $t_{\text {stat }}$ of the 5 (red), 10 (blue), 15 (magenta) and 20 (black) step delay RLS filters with a constant 0.7 seconds time delay (7 time steps). The dashed lines indicate the reference mean $\mu=0$ (blue), the positive accept/rejection threshold $T_{r}=3.5$ (red) and the negative accept/rejection threshold $-T_{r}=-3.5$ (yellow)

Lets look at example two, we simulate with a constant timed delay of 0.8 seconds ( 8 
time steps). The closest step size to 8 time step is 10, hence the 10 step RLS filter gets selected.
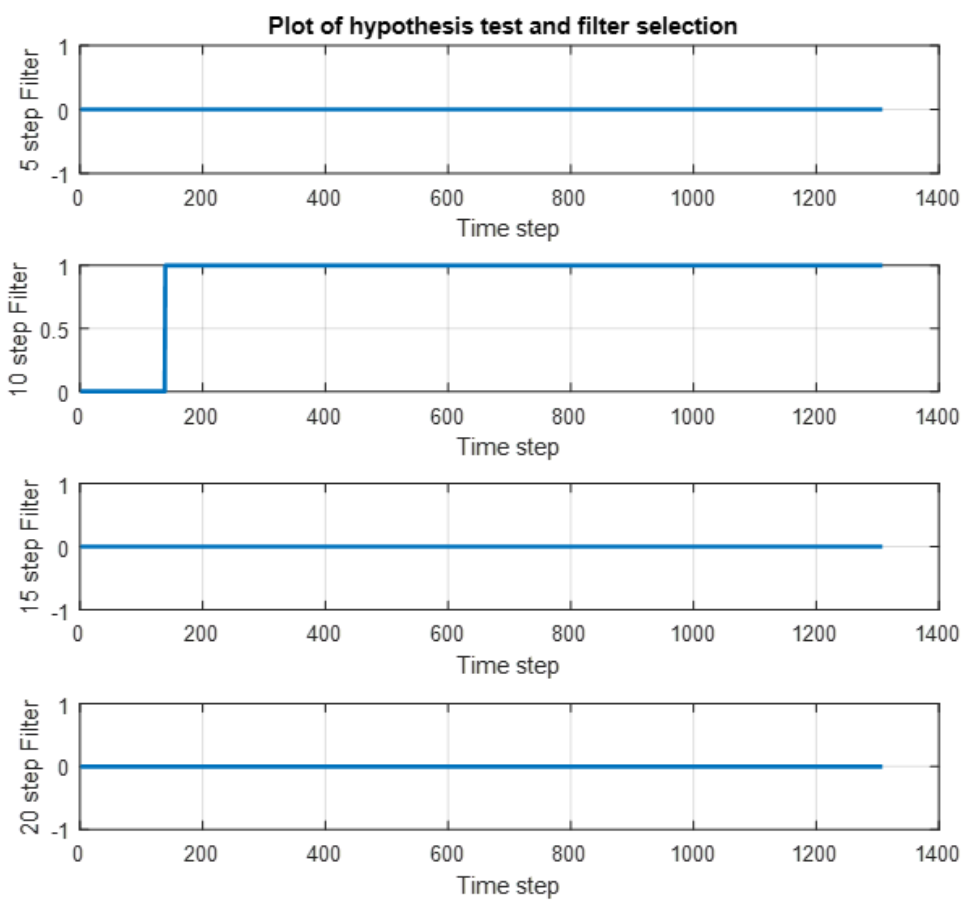

Figure 4.20: Example 2: Hypothesis test selection of 10 step RLS filter for a 0.8 seconds timed delay. 1 indicates that the corresponding RLS filter is selected. 0 indicates that the corresponding RLS filter is not selected.

At $t=140$, approximately 14 seconds, the 10 step RLS filter accepts the hypothesis and it is selected. The delay is then approximated to be 1 second and the target waypoint is approximated to the estimate $\hat{\Theta}_{10}\left(\theta_{10 x}(t)=2016.98, \theta_{10 y}(t)=4997.89\right)$ of the 10 step RLS filter. Just as in the case of the 7 step delay, the approximation is possible because of the selection of significance level $\alpha$ as 0.002 .

Figure 4.21 illustrates the test statistic for the 5, 10, 15 and 20 step RLS filters. We can observe that the test statistic of the 10 step delay RLS filter is in the acceptance region as illustrated in the figure. The test statistic of the 5, 15 and 20 step delay RLS filter are outside the acceptance region. Once again it can be 
observed how that the 10 step delay RLS filter is selected as its step size is closest to the 8 time step delay.

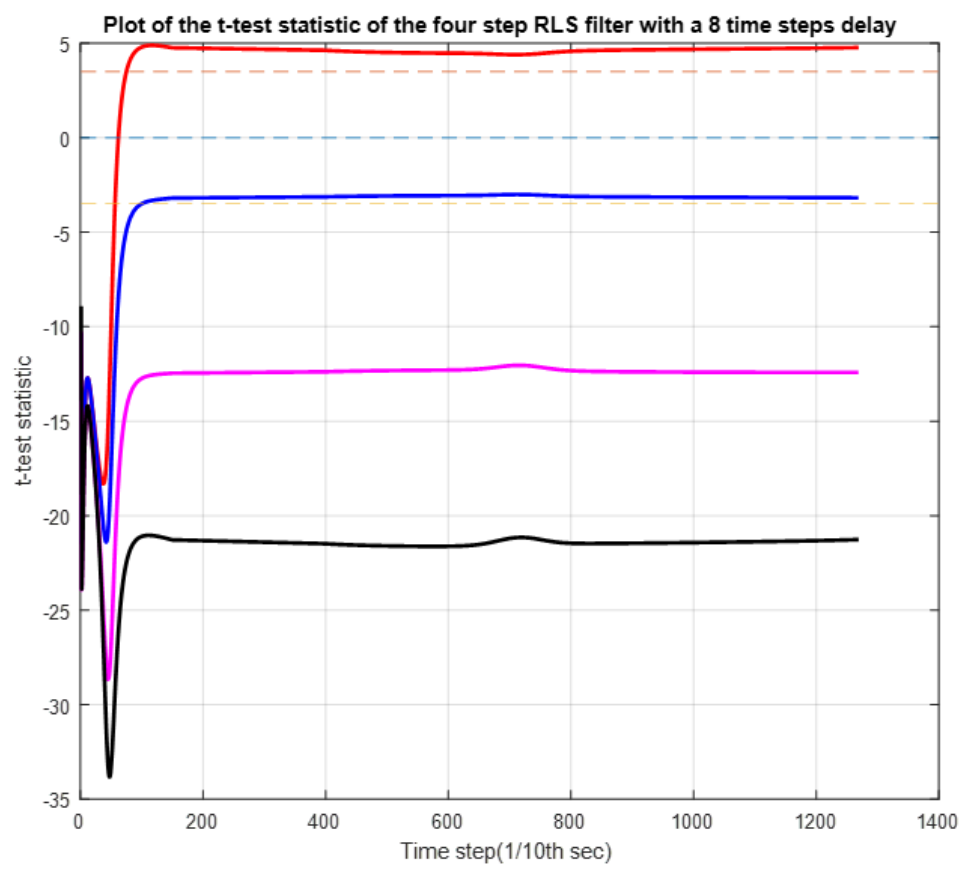

Figure 4.21: Example 2: T-test statistic $t_{\text {stat }}$ of the 5 (red), 10 (blue), 15 (magenta) and 20 (black) step RLS filters with a 0.8 seconds time delay (8 time steps). The dashed lines indicate the reference mean $\mu=0$ (blue), the positive accept/rejection threshold $T_{r}=3.5$ (red) and the negative accept/rejection threshold $-T_{r}=-3.5$ (yellow).

If in a case of multiple delays where one of the delays is unmatched with a RLS filter, a similar approximation is done such that the RLS filter with an assumed closest to the actual delay time step gets selected. For example three, we implement 0.7 seconds, 1 second and 2 seconds delays in the following sequence: $20 \leq t \leq 500$ delay $=1$ second (10 time steps), $500<t \leq 900$ delay $=0.7$ seconds $(7$ time steps $)$ and $t>900$ delay $=2$ seconds (20 time steps). The level of significance $\alpha=0.002$ and $T_{r}=3.5$. 

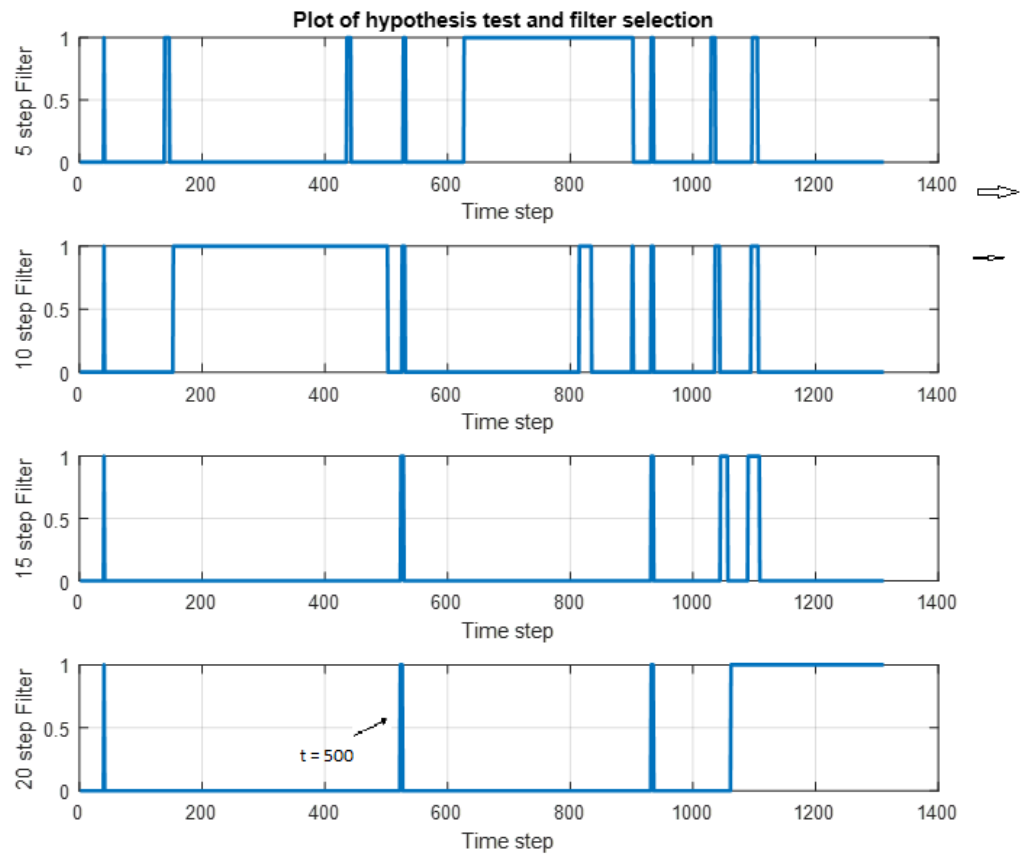

Figure 4.22: Example 3: Hypothesis test selection of RLS filters in a case of multiple delays and unmatched delays. 1 indicates that the corresponding RLS filter is selected. 0 indicates that the corresponding RLS filter is not selected.

In figure 4.22 one can see the selection of multiple filters at different time steps. Figure 4.23 illustrates where the test statistic of the RLS filters are located, either in the acceptance or rejection region. The selection of multiple RLS filters at the same time can be observed to occur at different time steps. For example in figure 4.22 at $t=500$ steps when the delay changes from 10 time steps, one can see that all four filers are selected. 


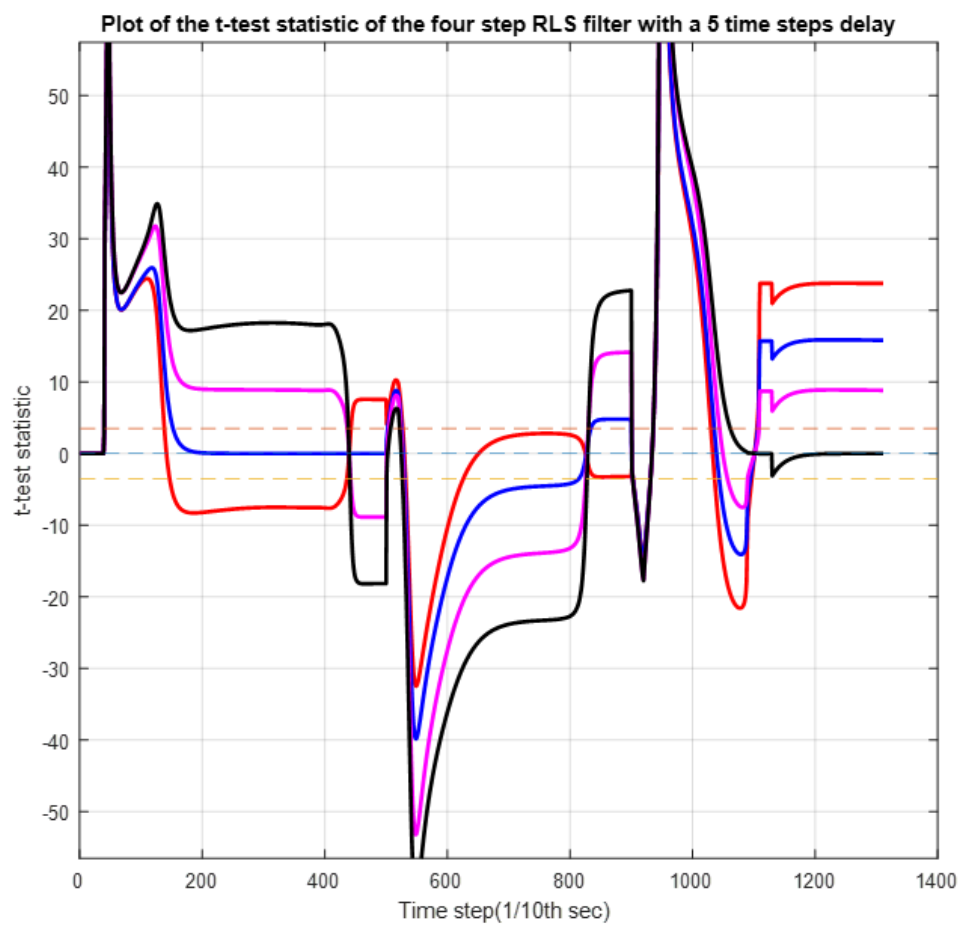

Figure 4.23: Example 3: T-test statistic $t_{\text {stat }}$ of the 5 (red), 10 (blue), 15 (magenta) and 20 (black) step RLS filters with 1 second (10 time steps), 0.7 seconds (7 time steps) and 2 seconds (20 time steps) time delay ( 7 time steps). The dashed lines indicate the reference mean $\mu=0$ (blue), the positive accept/rejection threshold $T_{r}=3.5$ (red) and the negative accept/rejection threshold $-T_{r}=$ -3.5 (yellow)

We also observe multiple RLS filter selection between $t=900$, when the delay changes from 10 time steps, and at $t=1060$, when the RLS filters converge to their final values. This multiple RLS filter selection activates the second selection criteria $J$ defined by eq 4.15 which forces the selection of the RLS filter with the least mean deviation of the prediction errors. The final output is illustrated in figure 4.23 . When a RLS filter is selected for just one time step, the selection is ignored. 

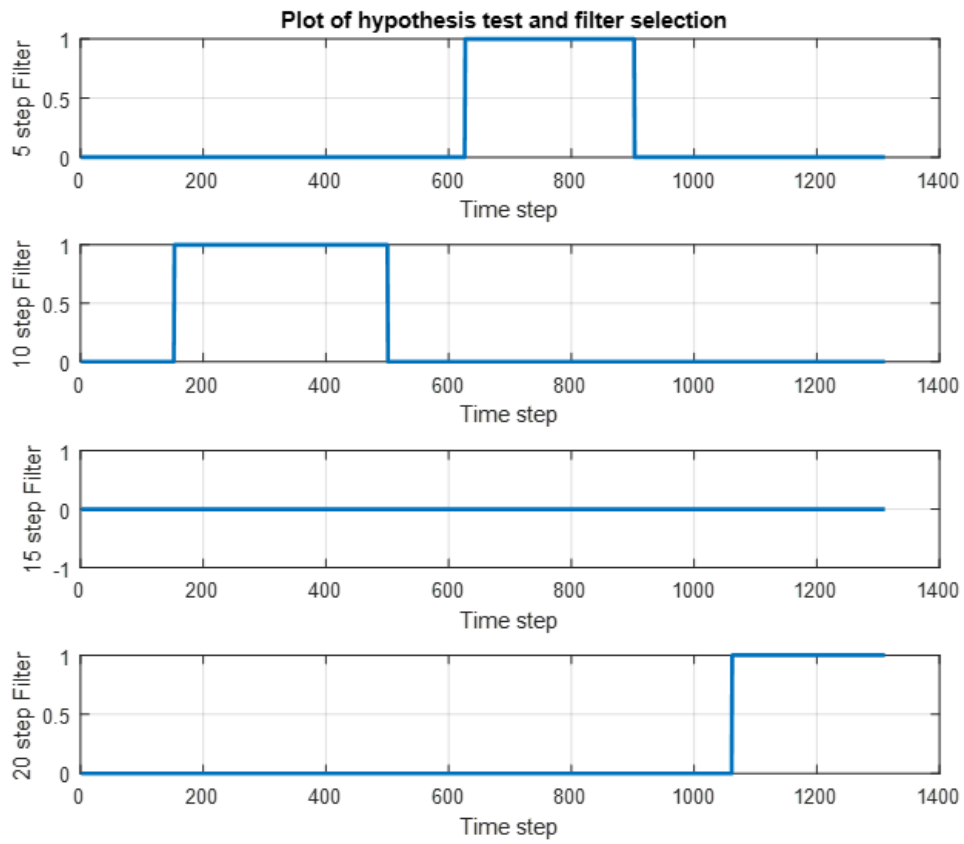

Figure 4.24: Example 3: Final output of hypothesis test selection of RLS filters with a 1 second, 0.7 seconds and 2 seconds time delay. 1 indicates that the corresponding RLS filter is selected. 0 indicates that the corresponding RLS filter is not selected.

At $t=160$, approximately 16 seconds, the 10 step RLS filter accepts the hypothesis and it is selected. The delay is then approximated to be 1 second and the target waypoint is approximated to the estimate $\hat{\Theta}_{10}\left(\theta_{10 x}(t)=2000, \theta_{10 y}(t)=5000\right)$ of the 10 step delay RLS filter. The 10 step delay RLS filter is deselected at $t=501$ time steps when the delay changes to 0.7 seconds ( 7 time steps). It takes the RLS filters 130 time steps (13 seconds) to converge. At $t=630$, the 5 step delay RLS filter is selected as discussed in example one. The delay is then approximated to be 0.5 seconds and the target waypoint is approximated to the estimate $\hat{\Theta}_{5}$ $\left(\theta_{5 x}(t)=2022.01, \theta_{5 y}(t)=4999.59\right)$ of the 5 step delay RLS filter. At $t=900$, when the delay changes to 2 seconds (20 time steps), the 5 step delay RLS filter is deselected and 160 time steps later, the 20 step delay RLS filter is selected. The delay is then approximated to be 2 seconds and the target waypoint is approximated 
to the estimate $\hat{\Theta}_{20}\left(\theta_{20 x}(t)=2000, \theta_{20 y}(t)=5000\right)$ of the 20 step RLS filter.

\subsection{Delay Compensation}

Once a RLS filter is selected, then one knows the estimated time delay and the target way point. Recall the target waypoint is the parameter estimate of the selected RLS filter $\hat{\Theta}_{i}$ in eq 3.62 .

$$
\hat{\Theta}_{i}=\left[\begin{array}{c}
\hat{\theta}_{i x} \\
\hat{\theta}_{i y}
\end{array}\right]
$$

While the selected RLS filter remains selected, with the estimate parameter $\Theta_{i}$ of the selected filter, the UAV can compute its heading $\psi_{\text {uav }}(t)$ to the estimated target waypoint from its position at each time step $t$. The autonomous heading $\psi_{\text {uav }}(t)$ becomes the input to the UAV controller and the UAV is now able to autonomously navigate its way to the target waypoint. The autonomous heading $\psi_{\text {uav }}(t)$ is computed as (see figure 3.1):

$$
\psi_{\text {uav }}(t)=\tan ^{-1}\left(\frac{\hat{\theta}_{i y}-y_{\text {uav }}(t)}{\hat{\theta}_{\text {ix }}-x_{\text {uav }}(t)}\right)
$$

where $\hat{\theta}_{i x}$ and $\hat{\theta}_{i y}$ are the xy-coordinate of the target waypoint, $x_{\text {uav }}(t)$ and $y_{\text {uav }}(t)$ are the xy-coordinates of the UAV location at time $t$. Note that the autonomous heading $\psi_{\text {uav }}(t)$, is calculated in the same way as the delayed teleoperator heading command $\psi_{\text {command }}(t)$ from eq 2.20 . Once the UAV learns the delay and waypoint, a signal is activated that triggers the switch that changes the input into the UAV controller from the delayed teleoperator heading command $\psi_{\text {command }}(t)$ to the autonomous heading $\psi_{\text {uav }}(t)$. To include a delay compensator, the system in chapter 2 (see figure 2.3 can be modified to: 


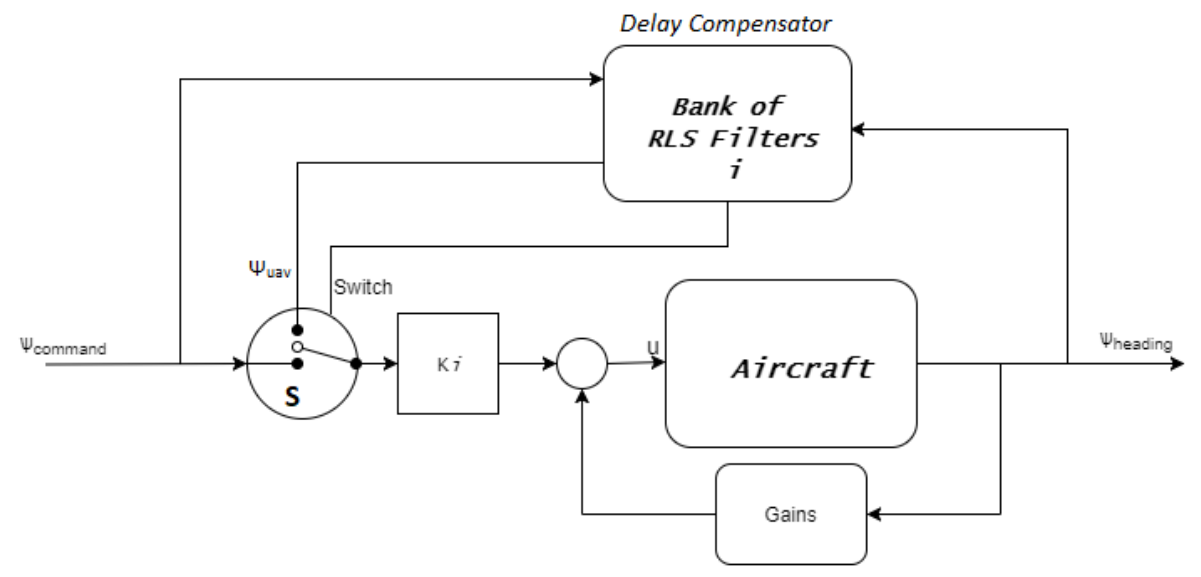

Figure 4.25: Modified Aircraft system for autonomous navigation

The delay compensator is a combination of an autonomous heading generator and a bank of RLS filters with each filter matched to a specific time delay. Once a RLS filter is selected, the autonomous heading generator uses the target waypoint estimates $\Theta_{i}$ of the selected RLS filter to generate autonomous heading command $\psi_{\text {uav }}(t)$. A signal is transmitted to the switch $S$ which triggers a change in input to the UAV controller from the delayed teleoperator commands $\psi_{\text {command }}(t)$ to the autonomous heading command $\psi_{\text {uav }}(t)$ (from the delay compensator). With the execution of the autonomous heading command $\psi_{\text {uav }}(t)$, the UAV performs an autonomous navigation to the target waypoint without delays.

If no RLS filter is selected (no delay detected), the input to the UAV controller is switched from the delay compensator to the teleoperator commands $\left(\psi_{\text {command }}(t)\right)$. The delay compensator keeps track of the operator commands so that if there are any changes to target waypoint or to the delay, the new delay and waypoint are learnt and adjustments are made accordingly.

\section{Demonstration of delay Compensation}

Figure 4.26 illustrates the trajectory of a UAV receiving delayed input commands from a teleoperator as it travels to target waypoints $A$ to $F$. Lets demonstrate how 
delays can be compensated for using a delay compensator.

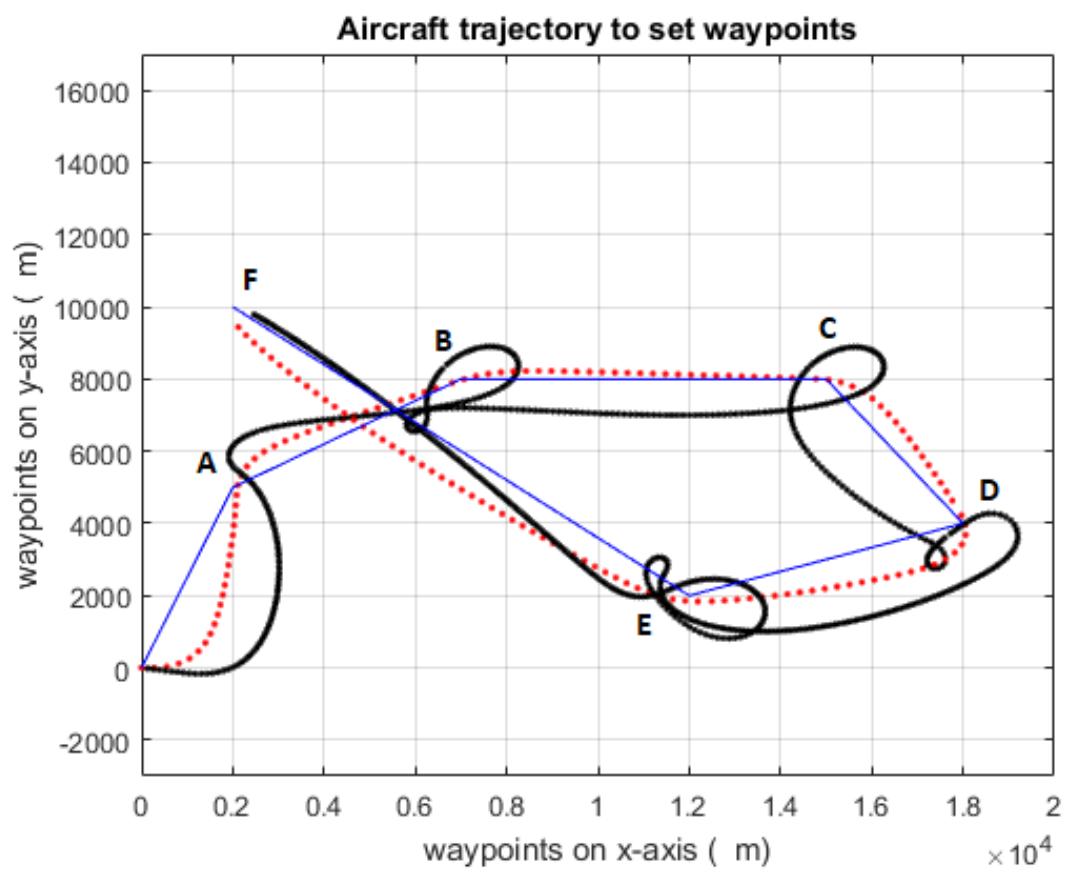

Figure 4.26: Trajectory of a UAV receiving input commands $\psi_{\text {command }}$ subjected to a 10 seconds time delay. The red dotted line indicates the aircraft trajectory with no delay, the blue line is the straight line between waypoints and the black line indicates the UAV trajectory subjected to time delay.

We simulate the UAV with delayed input commands $\psi_{\text {command }}(t)$ as it travels to the target waypoint at $A(2000,5000)$. For clarity, we simulate with time delays of 20 seconds (200 time steps), 30 seconds (300 time steps) and 60 seconds (600 time steps). Recall from chapter two, that a 30 seconds (300 time steps) delay in the input command $\psi_{\text {command }}(t)$ results in an uncontrollable UAV system illustrated in figure 2.21. Similarly, a 60 seconds (600 time steps) time delay will cause the the UAV to become uncontrollable. The UAV is said to have reached the target waypoint when it comes within the user defined radius of the target waypoint, in this case, waypoint $A$ and radius $(R=500)$. Four RLS filters are designed to track past UAV coordinates in 100, 200, 300 and 600 time step delays. 
Compensation for 20 seconds time delay.
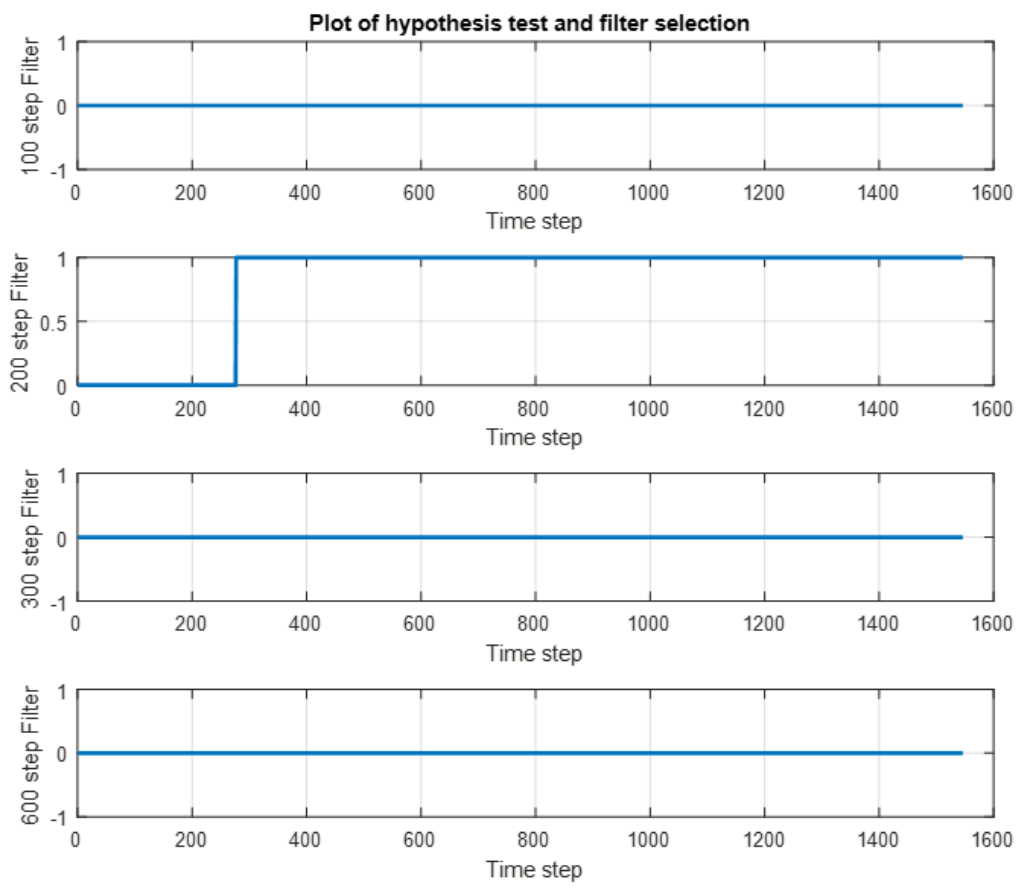

Figure 4.27: Hypothesis test selection of the 200 step delay RLS filter.

At time step $t=277$ which is approximately 28 seconds, the 200 step RLS filter accepts the null hypothesis and is selected. The delay is learned as a 200 time steps delay (20 seconds) and the target waypoint is selected to be the estimate $\Theta_{200}$ $\left(\theta_{200 x}(t)=2000, \theta_{200 y}(t)=5000\right)$. The autonomous heading $\psi_{\text {uav }}$ is then calculated with the selected target waypoint from eq 4.17. This activates the switch to change the input to the UAV controller from the 20 seconds delayed teleoperator heading $\psi_{\text {command }}(t)$ to the autonomous heading $\psi_{\text {uav }}$. In figure 4.28 comparison is made between the delayed trajectory and the compensated trajectory of the UAV. 


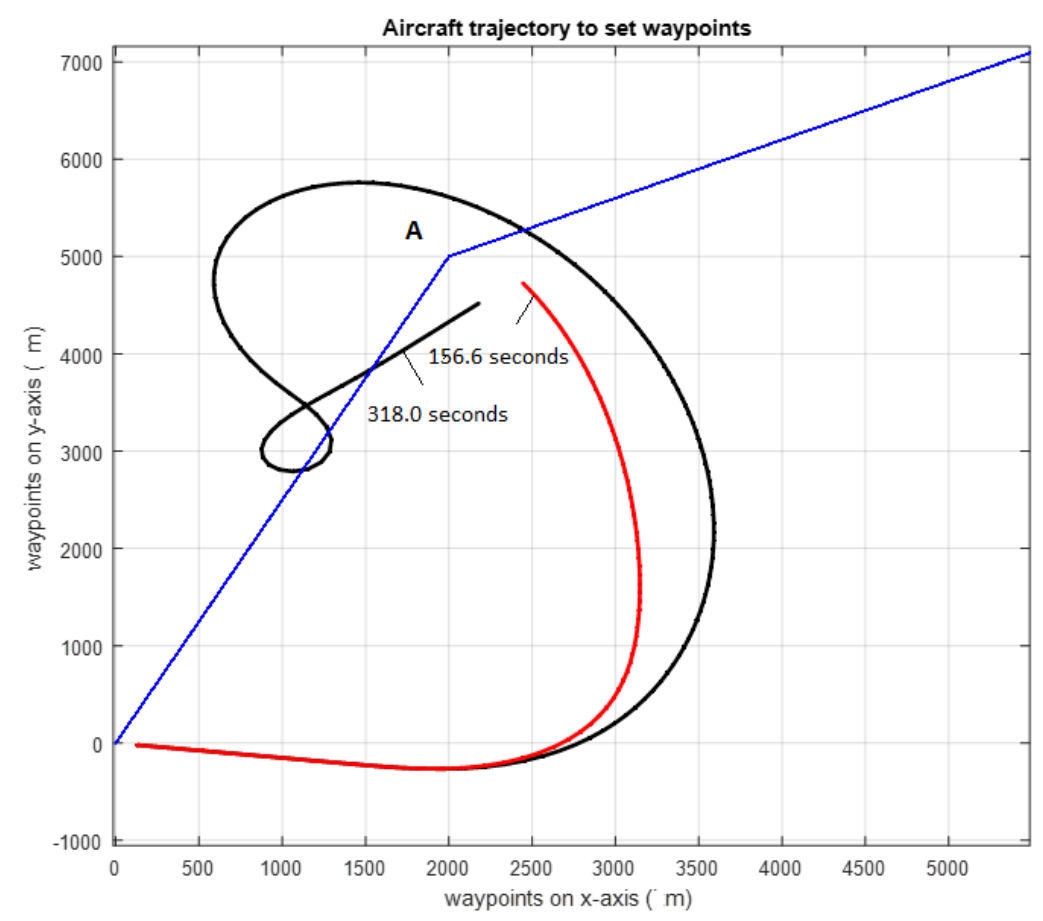

Figure 4.28: Compensation for a 20 seconds delay in input commands. The blue line is the straight line between waypoints and the black line indicates the UAV trajectory subjected to 20 seconds time delay, the red line indicates the UAV trajectory with delay compensation.

Once the switch to autonomous heading $\psi_{\text {uav }}$ is made, the UAV then executes an autonomous navigation to the estimated target waypoint by executing the autonomous heading $\psi_{\text {uav }}(t)$. In figure 4.28 we can see the trajectory of the UAV with delayed input command as it misses the waypoint. We can also see the increase in displacement between the delayed trajectory and the straight line between waypoints, indicating more distance and time travelled with delayed inputs. With autonomous navigation, we can observe that the UAV reaches the waypoint without any target miss and we also observe that the displacement is reduce indicating less travel distance and less travel time. With the delay, the UAV reached the waypoint in 318.0 seconds. With delay compensation, the UAV reached its waypoint in 156.6 seconds. Which is approximately a 50 percent time reduction. 
Compensation for 30 seconds time delay.
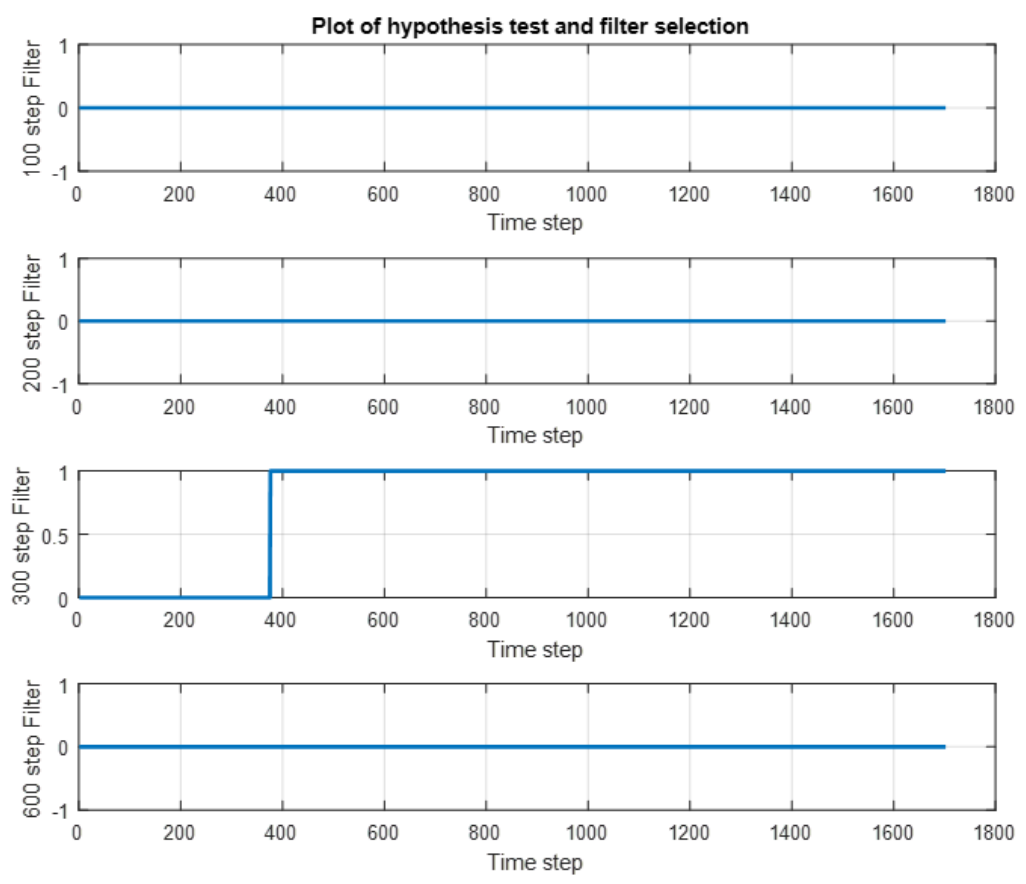

Figure 4.29: Hypothesis test selection of the 300 step delay RLS filter.

At time step $t=376$ which is approximately 38 seconds, the 300 step delay RLS filter accepts the null hypothesis and is selected. The delay is learned as 300 time steps (30 seconds) and the target waypoint is selected to be the estimate $\Theta_{300}\left(\theta_{300 x}(t)=\right.$ $\left.2000, \theta_{300 y}(t)=5000\right)$. 




Figure 4.30: Compensation for a 30 seconds delay in input commands. The blue line is the straight line between waypoints and the black line indicates the UAV trajectory subjected to 30 seconds time delay, the red line indicates the UAV trajectory with delay compensation.

From figure 4.30 we can observe that with the 30 seconds delay, the UAV becomes uncontrollable and the UAV keeps going in circles about the waypoint but does not reach the waypoint. When the delay is learned and a switch to autonomous navigation is made, the UAV reaches the waypoint without a target miss. We can also observe the travelled distance which also indicates how much time is spent travelling. The simulation with 30 seconds delay was stopped at $t=6788$ time steps (680 seconds) and at this time, the UAV did not get to the waypoint. With the switch, the UAV reached the waypoint in 1734 time steps (173 seconds).

Compensation for 60 seconds time delay. 

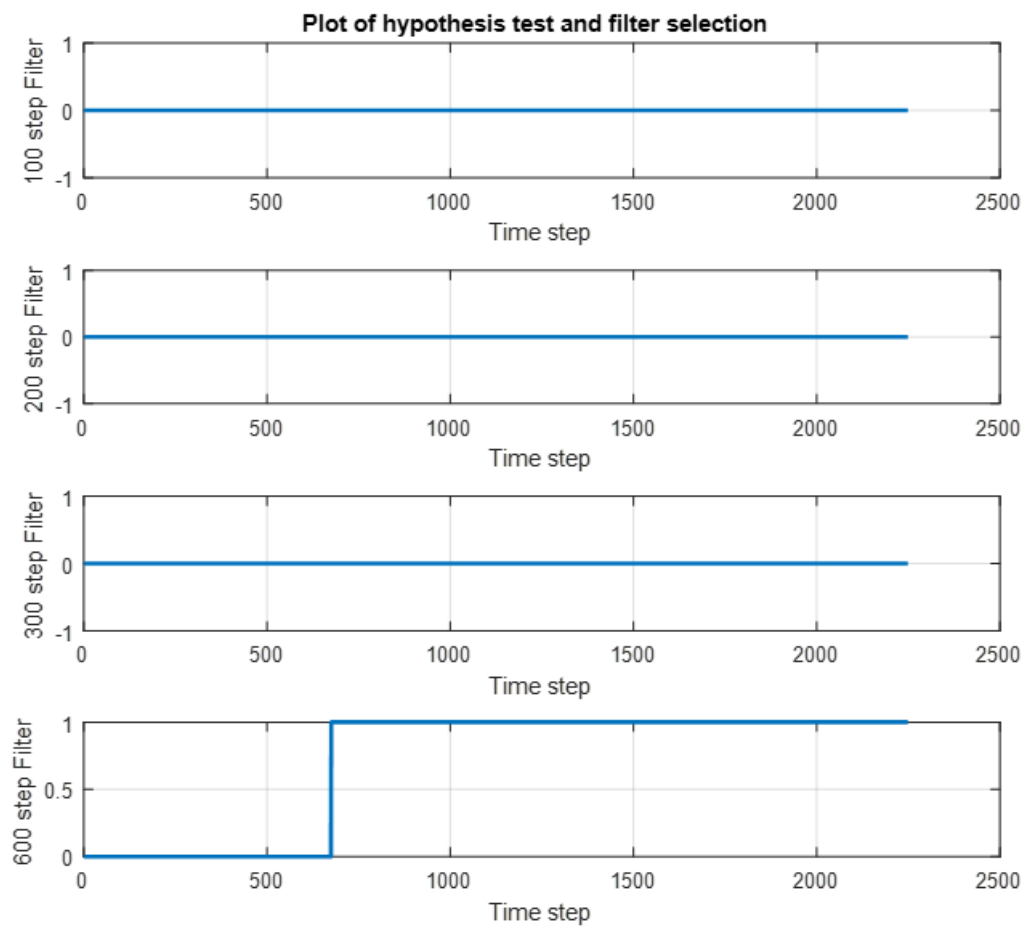

Figure 4.31: Hypothesis test selection of the 600 step delay RLS filter.

At time step $t=675$ which is approximately 68 seconds, the 600 step RLS filter accepts the null hypothesis and is selected. The delay is learned as a 600 time step delay (60 seconds) and the target waypoint is selected to be the estimate $\Theta_{600}$ $\left(\theta_{600 x}(t)=2000, \theta_{600 y}(t)=5000\right)$. 




Figure 4.32: Compensation for a 60 seconds delay in input commands. The blue line is the straight line between waypoints and the black line indicates the UAV trajectory subjected to 60 seconds time delay, the red line indicates the UAV trajectory with delay compensation.

From figure 4.32, just like the case of the 30 seconds delay, we can observe that with the 60 seconds delay, the UAV becomes uncontrollable and the UAV keeps going in even wider circles about the waypoint but does not reach the waypoint. When the delay is learnt and a switch to autonomous navigation is made, the UAV reaches the waypoint without a target miss. The simulation with 60 seconds delay was also stopped at $t=7501$ time steps (750 seconds) and at this time, the UAV did not get to the waypoint. With the switch, the UAV reached the waypoint in 2253 time steps (225 seconds). 


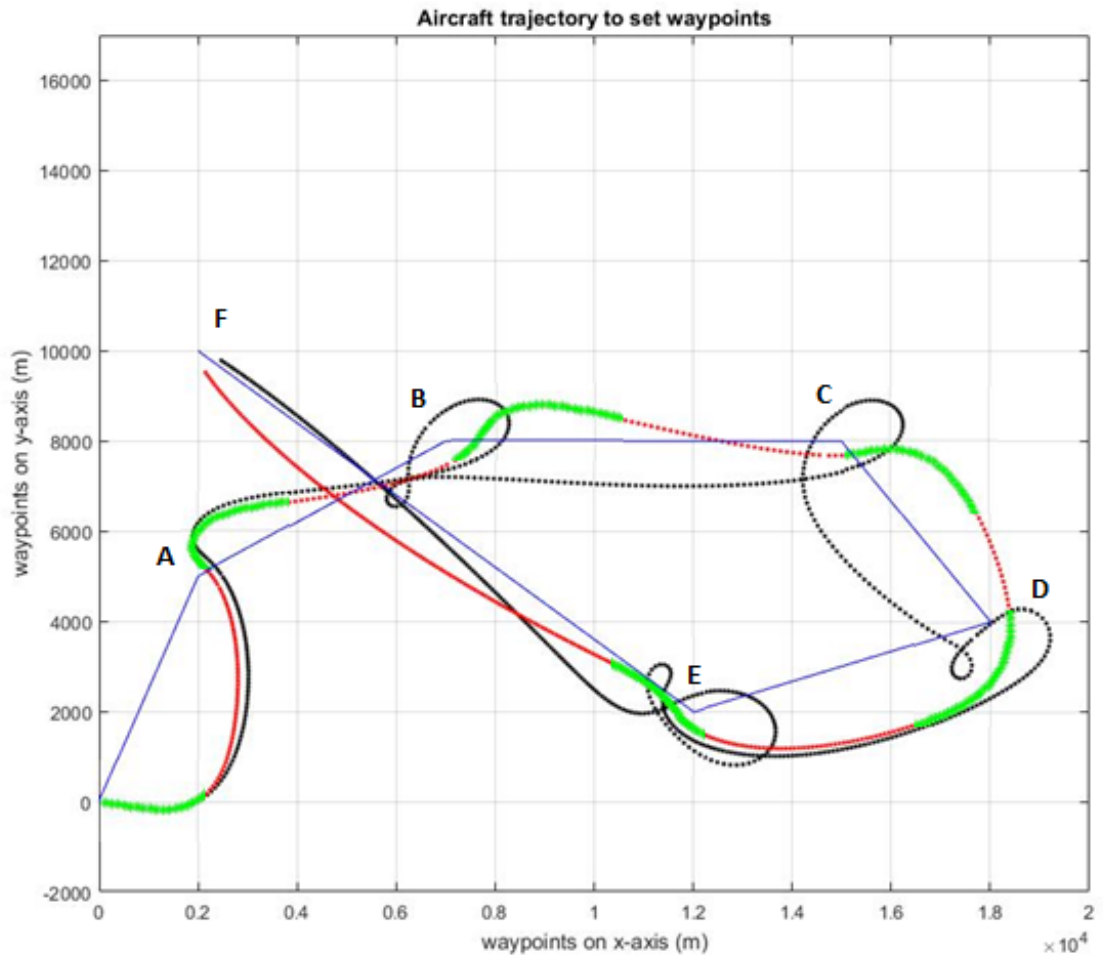

Figure 4.33: Compensation for a $10 s$ delay in input commands as the UAV moves from $A$ to $F$. The blue line is the straight line between waypoints and the black line indicates the UAV trajectory subjected to 10 seconds time delay, the red line indicates the UAV trajectory with delay compensation. The green sections indicated the periods when UAV is actively controlled by the operator.

Figure 4.33 illustrates the UAV trajectory with a 10 seconds delayed $\psi_{\text {command }}(t)$ as the UAV moves from one waypoints $A$ to $F$. In the delayed trajectory, we see that the UAV misses the waypoints and there is some manoeuvring to try get back to the waypoints, but with the delay compensator and auto-navigation, there is no waypoint miss. When the UAV reaches its target waypoint, the switch changes to the teleoperator and checks for changes in delay or changes in in target waypoint as is the case where the waypoint changed from $A$ to $B$. At this point the prediction errors of RLS filters are not close to zero as such no RLS filers are seleccted. The UAV begins to learn the new delay and new waypoint and the UAV goes through the process of auto-navigation. 
The examples with 10, 20, 30 and 60 seconds time delay demonstrate that with a bank of RLS filters each matched to a time delay, the UAV can learn the delay in the input commands and also learn the target waypoints. The UAV can then compensate for the delay by computing its autonomous heading command $\psi_{\text {uav }}(t)$ from the UAVs coordinates at each time step. 


\section{Chapter 5}

\section{Conclusion and Future Work}

\subsection{Conclusion}

The objective of this thesis is to develop a method of compensating for timed delays in unmanned aerial vehicles (UAVs). We formulated a recursive least squares (RLS) model for the waypoint and the assumed delay. We defined a bank of RLS filters each matched to specific time delay which indicates how many time steps into the past UAV coordinates individual RLS filters track. A close to zero prediction error determines which filter has the best estimate of the target waypoint and hence the delay.

Hypothesis testing for filter selection was implemented to reduce control errors and heuristic approximations. A two tailed t-test was used to define a criteria by which filters are selected within a user defined level of significance $\alpha$.

Once the delay and target waypoints are learned, the UAV computes its heading $\psi_{\text {uav }}$ and by executing the self computed heading $\psi_{\text {uav }}$ the UAV performs autonomous navigation towards the learned target waypoint.

Simulations using time delays of 20, 30 and 60 seconds show how autonomous navigation can be used to compensate for time delays in the UAV system. Results 
from this method of compensation show that compensation by autonomous navigation increased target reach accuracy and precision. The UAV with delayed inputs misses the target waypoint but with the delay compensation the UAV reaches the target waypoints without any target miss.

With autonomous navigation there was a reduction in travel distance. The simulations show that the UAV with delayed inputs travel for longer distances as illustrated by the displacement between the UAV trajectory and the straight line between waypoints. With delay compensation this travel distance was reduced.

With autonomous navigation there was also a reduction in travel time. The UAV with delayed inputs travel for longer time than when the delay is being compensated for. For example, in the case of the 20 seconds delay, the UAV travelled for approximately 320 seconds before it reached a target waypoint A (see figure 4.28), but with delay compensation, the UAV travelled for approximately 160 seconds.

In situations where a teleoperator loses control of the UAV due to time delays as was the case when we simulated with a 30 seconds time delay (see figure 4.30), autonomous navigation restores control of the UAV and this enables the UAV to reach the waypoint without a target miss and in a more coordinated trajectory.

\subsubsection{Future Work}

The work presented in this thesis opens up new directions for research in unsupervised learning. In the future the bank of RLS filters can be designed to include a filter whose assumed delay can be varied so that in cases where the time delay does not match to a specific filter as discussed in chapter 4 , the filter with variable assumed delay can adapt to match with the time delay. This will further increase accuracy in learning the waypoint and the delay. 


\section{List of References}

[1] E. J. Feron and E. N, Aerial robotics. Springer, 2008.

[2] R. Carloni, V. Lippiello, M. D'auria, M. Fumagalli, A. Y. Mersha, S. Stramigioli, and B. Siciliano, "Robot vision: Obstacle-avoidance techniques for unmanned aerial vehicles," IEEE Robotics \&3 Automation Magazine, vol. 20, no. 4, pp. 22-31, 2013.

[3] J. L. Adams, "An investigation of the effects of the time lag due to long transmission distances upon remote control. phase i tracking experiments," tech. rep., National Aeronautics and Space Administration Washington DC, 1961.

[4] T. Sheridan and W. Ferrell, "Remote manipulative control with transmission delay," IEEE Transactions on Human Factors in Electronics, vol. 1, no. 4, pp. 25-29, 1963.

[5] M. E. Lloyd, "The effect of control and display lag on uas internal pilot manual landing performance," Master's thesis, Embry- Riddle Aeronautical University, Daytona Beach, Florida, 2012.

[6] S. A. Dougherty, An examination of latency and Degradation Issues in Unmanned Combat Vehicle Environments. PhD thesis, Air Force Inst of Tech Wright-Patterson AFB, Ohio, 2002.

[7] Z. W. H. Dong and H. Gao, "Robust filtering for a class of nonlinear networked systems with multiple stochastic communication delays and packet dropouts," IEEE Transactions on Signal Processing, vol. 4, no. 58, pp. 1957-1966, 2010.

[8] E. P. Ahmet Onat, Teoman Naskali and O. Mutluer, "Control over imperfect networks: Model-based predictive networked cotrol system," IEEE Transactions on Industrial Electronics, vol. 3, no. 58, pp. 905-913, 2011.

[9] J. K. Yun-Bo Zhao and G.-P. Liu, "Error bounded sensing for packet-based networked control systems," IEEE Transactions on Industrial Electronics, vol. 5, no. 58, pp. 1980-1989, 2011. 
[10] J. Yoo and K. H. Johansson, "Learning communication delay patterns for remotely controlled uav networks," IFAC-PapersOnLine, vol. 50, no. 1 , pp. 13216-13221, 2017.

[11] W. T. Nelson, L. J. Hettinger, M. W. Haas, C. J. Russell, J. S. Warm, W. N. Dember, and T. A. Stoffregen, "Compensation for the effects of time delay in a helmet-mounted display: perceptual adaptation versus algorithmic prediction," SPIE's 1995 Symposium on OE/Aerospace Sensing and Dual Use Photonics, vol. 2465, pp. 154-165, 1995.

[12] A. K. Bejczy and W. S. Kim, "Predictive displays and shared compliance control for time-delayed telemanipulation," IEEE International Workshop on Intelligent Robots and Systems, Towards a New Frontier of Applications, pp. 407-412, 1990.

[13] J. S.C and P. P., "Constrained mpc algorithm for uncertaine time-varying systems with state delay," IEEE Transactions on Automatic Control, vol. 2, no. 50, pp. 257-263, 2005.

[14] A. G. Mutambara, Decentralized estimation and control for multisensor systems. CRC press, 1998.

[15] B. Friedland, Control Systems Design: An introduction to State-Space Methods. Dover Publishers, 2012.

[16] K. Ogata and Y. Yang, Modern control engineering, vol. 4. Prentice hall India, 2002.

[17] H. M. Schwartz, Multi-Agent Machine Learning: A Reinforcement Approach. John Wiley \& Sons, 2014.

[18] B. D. Anderson and J. B. Moore, "Optimal filtering," Englewood Cliffs, vol. 21, pp. 22-95, 1979.

[19] F. Chowdury, "Kalman filter with hypothesis testing: A tool for estimating uncertain parameters," Circuits, Systems and Signal Processing, vol. 15, no. 3, pp. 291-311, 1996.

[20] J. C. F. Chowdury and J. Aravena, "Power detection and state estimation using kalman filter with hypothesis testing," IEEE Transactions on Power delivery, vol. 6, no. 3, pp. 1025-1030, 1991. 
[21] A. S. Campbell, "Improvements to multiple model adaptive control: Hypothesis test switching and a modified model arrangement," Master's thesis, Carleton University, Ottawa, Ontario, 2005.

[22] D. Wackerly, W. Mendenhall, and R. L. Scheaffer, Mathematical statistics with applications. Cengage Learning, 2014.

[23] R. J. Larsen, M. L. Marx, et al., An introduction to mathematical statistics and its applications, vol. 2. Prentice-Hall Englewood Cliffs, NJ, 1986. 